A REVIEW OF UNIFIED A POSTERIORI FINITE ELEMENT ERROR CONTROL

\author{
By \\ C. Carstensen \\ M. Eigel \\ C. Löbhard \\ and \\ R.H.W. Hoppe
}

IMA Preprint Series \# 2338

(October 2010)

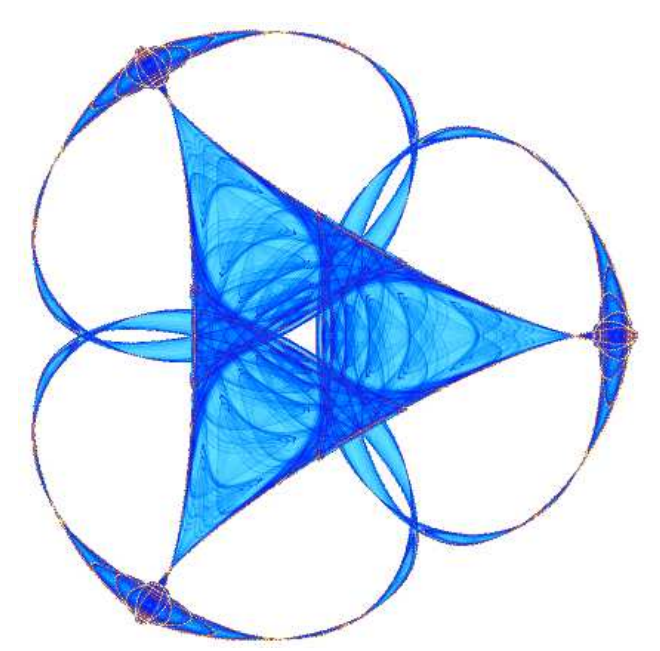

INSTITUTE FOR MATHEMATICS AND ITS APPLICATIONS

UNIVERSITY OF MINNESOTA 400 Lind Hall

207 Church Street S.E.

Minneapolis, Minnesota 55455-0436

Phone: 612-624-6066 Fax: 612-626-7370

URL: http://www.ima.umn.edu 


\title{
A REVIEW OF UNIFIED A POSTERIORI FINITE ELEMENT ERROR CONTROL
}

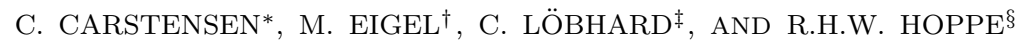

Abstract. This paper aims at a general guideline to obtain a posteriori error estimates for the finite element error control in computational partial differential equations.

In the abstract setting of mixed formulations, a generalised formulation of the corresponding residuals is proposed which then allows for the unified estimation of the respective dual norms. Notably, this can be done with an approach which is applicable in the same way to conforming, nonconforming and mixed discretisations. Subsequently, the unified approach is applied to various model problems. In particular, we consider the Laplace, Stokes, Navier-Lamé, and the semi-discrete eddy current equations.

Key words. A posteriori, error analysis, finite element method, nonconforming finite element method, mixed finite element method, adaptive algorithm, Poisson equation, Lamé equations, Stokes equations, Maxwell equations, unified a posteriori error analysis, discontinuous Galerkin, residual estimator

*Dep. of Math., Humboldt Universität zu Berlin, D-10099 Berlin, Germany, and Dep. of Comput. Sci. Eng., Yonsei University, Seoul 120-749, Korea. The work has been supported by the German National Science Foundation DFG within the Research Center MATHEON and by the WCU program through KOSEF (R312008-000-10049-0).,

${ }^{\dagger}$ Dep. of Math., Humboldt Universität zu Berlin, D-10099 Berlin, Germany.,

${ }^{\ddagger}$ Dep. of Math., Humboldt Universität zu Berlin, D-10099 Berlin, Germany.,

$\S$ Dep. of Math., University of Houston, Houston TX 77204-3008, USA, and Inst. of Math., University of Augsburg, D-86159 Augsburg, Germany. The author acknowledges support by the NSF grants DMS-0707602, DMS-0810176, DMS-0811153, DMS-0914788, by the German National Science Foundation within the Priority Programs SPP 1253, SPP 1506, by the German Federal Ministry for Education and Research (BMBF) within the projects BMBF-FROPT and BMBF-MeFreSim, and by the European Science Foundation (ESF) within the ESF Program OPTPDE. The first and the fourth author also want to express their sincere thanks to the IMA, University of Minnesota, for its hospitality during their stay in Fall 2010 on occasion of the IMA Special Year 'Simulating our complex world: modeling, computation, and analysis'. 


\section{Contents}

1 Introduction $\quad 3$

2 Well-Posed Continuous problems / Unified Notation of Model Problems 4



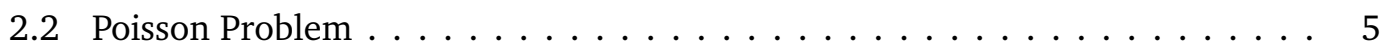

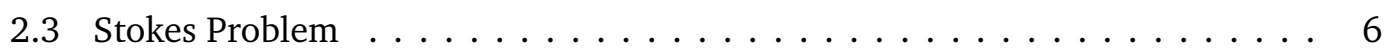

2.4 Lamé Problem . . . . . . . . . . . . . . . . . . . . 7

2.5 Eddy Current Problem . . . . . . . . . . . . . . . . . 8

3 Errors and Residuals $\quad 9$

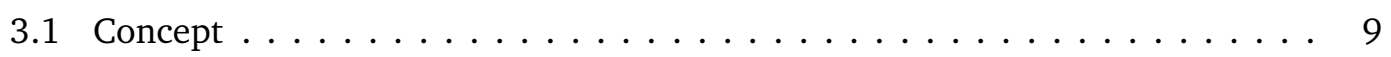

3.2 Hilbert Space Case . . . . . . . . . . . . . . . . . . . . 11

3.3 Poisson Problem . . . . . . . . . . . . . . . . . . . . . . . 12

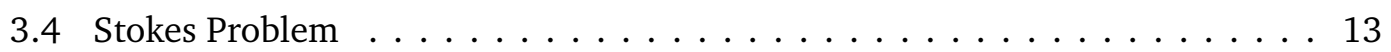

3.5 Lamé Problem . . . . . . . . . . . . . . . . . . . . . 13

3.6 Eddy Current Problem . . . . . . . . . . . . . . . . . . . . . 14

4 Finite Element Spaces 14

4.1 Notation . . . . . . . . . . . . . . . . . . . . . 15

4.2 Discrete Spaces and Basis Functions . . . . . . . . . . . . . . 15

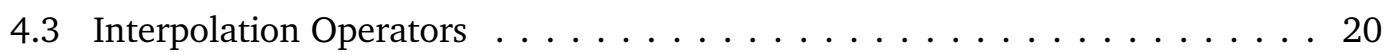

4.4 Discrete Problems . . . . . . . . . . . . . . . . . . 23

5 Abstract Dual Norm Estimates 24

5.1 Consistency Error Estimates . . . . . . . . . . . . . . . . . . 24

5.2 Explicit Equilibration Error Estimates . . . . . . . . . . . . . . . 27

6 Poisson Problem 31

6.1 Conforming Finite Element Methods . . . . . . . . . . . . . . . . . 31

6.2 Non-Conforming Finite Element Methods $\left(\mathrm{CR}_{1}\left(\mathscr{T}_{\ell}\right)\right) \ldots \ldots \ldots \ldots$

6.3 Mixed Finite Element Methods $\left(\mathrm{RT}_{0}\right) \ldots \ldots \ldots \ldots \ldots$

7 Stokes Problem 35

7.1 Mixed Conforming Finite Element Methods . . . . . . . . . . . . . . . 35

7.2 Non-Conforming Finite Element Methods . . . . . . . . . . . . . . . . 37

8 Lamé Problem $\quad 37$

8.1 Conforming Finite Element Methods . . . . . . . . . . . . . . 37

8.2 Non-Conforming Finite Element Methods . . . . . . . . . . . . . . . . 38

8.3 Mixed Finite Element Methods . . . . . . . . . . . . . . . . . . 38 
9 Eddy Current Problem $\quad 43$

9.1 Conforming Edge Element Methods . . . . . . . . . . . . . . . 43

9.2 Discontinuous Galerkin Methods . . . . . . . . . . . . . . . . . . . . 44

\section{Introduction}

Numerical simulation in engineering and science involves all kinds of errors ranging from the modeling of the problem to round-off errors. We are concerned with problems that can be formulated as the linear equation

$$
\mathscr{A}(p, u)=\ell
$$

in function spaces $Q$ and $V$ with $\mathscr{A}(p, u)$ and $\ell$ in $(Q \times V)^{*}$. This paper is devoted to the control of the discretisation error that arises from the fact that the (unknown) exact solution $(p, u) \in Q \times V$ is approximated by a discrete solution $\left(p_{\ell}, u_{\ell}\right)$ computed in a finite dimensional vector space $Q_{\ell} \times V_{\ell}$. The aim of a posteriori error control is the computation and justification of lower and upper error bounds for the unknown discretisation error $e:=(p, u)-\left(p_{\ell}, u_{\ell}\right)$. Apart from the standard case that the discrete spaces are subspaces of their infinite dimensional counterparts, we will also consider a violation of this inclusion in the case of non-conforming methods.

This paper is organized as follows. After some basic notations and definitions in this introductory section, a unifying formulation for different problem classes is introduced and applied to various examples in Section 2. Section 3 about the basic concepts of residual type error estimation is followed by a brief introduction to finite element spaces and interpolation operators in Section 4. The theoretical part is concluded by a compilation of crucial theorems in error estimation with proofs. In addition to the above statements, Sections 2 and 3 prepare applications discussed in detail in Sections 6 to 9.

Notation. In this paper, $a \lesssim b$ abbreviates $a \leq C b$ with some multiplicative mesh-size independent constant $C>0$ which only depends on the domain $\Omega$ and the shape (but not on the size) of finite element domains. Moreover, $C$ is independent of crucial parameters of the partial differential equation (PDE) such as the Lamé parameter $\lambda$ in the problem of linear elasticity below. Furthermore, $a \approx b$ abbreviates $a \lesssim b \lesssim a$.

There are different definitions of the differential operator curl which we use in the document. An overview is depicted in Table 1.1. Note that the curl of a function is always orthogonal to the gradient.

The Sobolev spaces of functions defined on a domain $\Omega \subset \mathbb{R}^{n}$ required for the formulation of the presented PDEs are as usual denoted by $H^{k}(\Omega)$ for the space of all functions in $L^{2}(\Omega)$ which allow weak derivation up to order $k$ and

$$
\begin{aligned}
H(\operatorname{div}, \Omega) & :=\left\{v \in L^{2}\left(\Omega ; \mathbb{R}^{n}\right) \mid \operatorname{div} v=\nabla \cdot v \in L^{2}(\Omega)\right\} \\
H(\operatorname{curl}, \Omega) & :=\left\{v \in L^{2}\left(\Omega ; \mathbb{R}^{3}\right) \mid \operatorname{curl} v=\nabla \wedge v \in L^{2}\left(\Omega ; \mathbb{R}^{3}\right)\right\}
\end{aligned}
$$




\begin{tabular}{c|cc} 
& $v: \Omega \subset \mathbb{R}^{n} \rightarrow \mathbb{R}$ & $v: \Omega \subset \mathbb{R}^{n} \rightarrow \mathbb{R}^{n}$ \\
\hline $\mathrm{n}=2$ & $\operatorname{Curl} v=\left(\begin{array}{c}\partial_{x_{2}} v \\
-\partial_{x_{1}} v\end{array}\right)$, & $\operatorname{curl} v=\partial_{x_{1}} v_{2}-\partial_{x_{2}} v_{1}$ \\
$\mathrm{n}=3$ & $\operatorname{Curl} v=\left(\begin{array}{c}\partial_{x_{2}} v-\partial_{x_{3}} v \\
\partial_{x_{3}} v-\partial_{x_{1}} v \\
\partial_{x_{1}} v-\partial_{x_{2}} v\end{array}\right)$, & $\operatorname{curl} v=\left(\begin{array}{c}\partial_{x_{2}} v_{3}-\partial_{x_{3}} v_{2} \\
\partial_{x_{3}} v_{1}-\partial_{x_{1}} v_{3} \\
\partial_{x_{1}} v_{2}-\partial_{x_{2}} v_{1}\end{array}\right)=\nabla \wedge v$.
\end{tabular}

Table 1.1: Overview of the different definitions of the curl differential operator.

Moreover, the spaces of functions with boundary conditions are written as

$$
\begin{aligned}
L_{0}^{2}(\Omega) & :=\left\{q \in L^{2}(\Omega) \mid \int_{\Omega} q d x=0\right\}, \\
H_{0}^{1}(\Omega) & :=\left\{v \in H^{1}(\Omega)|v|_{\partial \Omega}=0\right\}, \\
H_{0}(\operatorname{div}, \Omega) & :=\left\{v \in H(\operatorname{div}, \Omega)|v \cdot v|_{\partial \Omega}=0\right\}, \\
H_{0}(\operatorname{curl}, \Omega) & :=\left\{v \in H(\operatorname{curl}, \Omega)|(v \wedge v)|_{\partial \Omega}=0\right\} .
\end{aligned}
$$

where $v$ is the unit exterior normal vector on the boundary $\partial \Omega$ of $\Omega$.

Colon denotes the Euclidean scalar product of two matrices $A=\left(A_{j k}\right), B=\left(B_{j k}\right) \in$ $\mathbb{R}^{n \times n}$, that is, $A: B:=\sum_{j, k=1}^{n} A_{j k} B_{j k}$, the dyadic product of some vectors $a, b \in \mathbb{R}^{n}$ is denoted by $a \otimes b:=a \cdot b^{T}$ and the space of symmetric matrices in $\mathbb{R}$ is defined by

$$
\mathbb{R}_{\mathrm{sym}}^{n \times n}:=\left\{A \in \mathbb{R}^{n \times n}: A=A^{T}\right\} .
$$

Reliability and efficiency. The presented approach provides a general guideline in the derivation of reliable and efficient error estimators commonly denoted by $\eta$ or $\mu$. Any computable quantity in a numerical method is called error estimator. For the error $e$, we call an error estimator $\eta$ efficient, if

$$
\eta \lesssim\|e\|+\text { hot }_{\mathrm{eff}}
$$

and reliable, if

$$
\|e\| \lesssim \eta+\text { hot }_{\text {rel }} \text {. }
$$

Here "hot" denotes higher-order terms which usually are much smaller than $\eta$ and the error $e$, and which tend to zero with decreasing mesh size much faster. However, in general this may depend on the (unknown) smoothness of the solution and the (known) smoothness of the data.

\section{Well-Posed Continuous problems / Unified Notation of Model Problems}

\subsection{Generic Mixed Formulation}

The problems in this text can be written in terms of linear algebra as follows. The sets $Q$ and $V$ are real vector spaces with norms $\|\cdot\|_{Q}$ and $\|\cdot\|_{V}$ and the operator $\mathscr{A}$ maps 
linearly from $Q \times V$ to its dual $(Q \times V)^{*}$. A linear operator $\mathscr{A}$ is continuous, if and only if it is bounded. It is bijective if, for any $\ell \in(Q \times V)^{*}$, the equation $\mathscr{A}(p, u)=\ell$ has a unique solution $(\bar{p}, \bar{u})$. This defines an inverse mapping via $\mathscr{A}^{-1} \ell=(\bar{p}, \bar{u})$. The linear problem to seek $(p, u) \in Q \times V$ with $\mathscr{A}(p, u)=\ell$ is called well-posed if it is uniquely solvable and if $\mathscr{A}$ and the inverse mapping $\mathscr{A}^{-1}$ are continuous.

In Section 3, we show in the context of error analysis by residual estimation, that well-posedness of the problem is also essential for error estimation, although the unique solution might not be known.

All applications considered can be formulated in a mixed setting. A unified representation is obtained in terms of the (bi)linear continuous forms

$$
\begin{aligned}
a: Q \times Q \rightarrow \mathbb{R}, \\
c: V \times V \rightarrow \mathbb{R}, \\
\ell_{Q}: Q \rightarrow \mathbb{R}, \\
\ell_{V}: V \rightarrow \mathbb{R}
\end{aligned}
$$

plus a differential operator $\Lambda: V \rightarrow Q$. The space $V$ always is a $H^{1}, H$ (div) or $H$ (curl) space, $Q$ is usually an $L^{2}$ space and the bilinear form $b$ is defined via

$$
b: Q \times V \rightarrow \mathbb{R} \quad \text { with } \quad b(q, v):=a(q, \Lambda v) \quad \text { for all }(q, v) \in Q \times V .
$$

With this, we define

$$
\begin{aligned}
\mathscr{A}(p, u)(q, v) & :=a(p, q)+b(p, v)-b(q, u)+c(u, v), \\
\ell(q, v) & :=\ell_{Q}(q)+\ell_{V}(v) .
\end{aligned}
$$

Then, the problem allows the following split: Seek $(p, u) \in Q \times V$ such that

$$
\begin{array}{ll}
\forall q \in Q & a(p, q)-b(q, u)=\ell_{Q}(q), \\
\forall v \in V & b(p, v)+c(u, v)=\ell_{V}(v) .
\end{array}
$$

\subsection{Poisson Problem}

For a function $f \in L^{2}(\Omega)$, the Poisson problem reads: Seek $u \in H^{2}(\Omega)$ with

$$
\Delta u=f \text { in } \Omega \quad \text { and }\left.\quad u\right|_{\partial \Omega}=0 .
$$

The Poisson equation arises in a variety of physical phenomena with the density $u$ of some quantity in equilibrium, such as a chemical concentration, temperature or electrostatic potential.

In order to obtain error estimators of (2.3) with arbitrary data $f$, it is possible to examine the mixed formulation of the Poisson problem with two partial differential equations of first order instead of one equation of second order, namely, seek a tuple $(p, u) \in Q \times V:=$ $L^{2}\left(\Omega ; \mathbb{R}^{n}\right) \times H_{0}^{1}(\Omega)$, such that

$$
\nabla u=p \quad \text { and } \quad-\operatorname{div} p=f .
$$


For the Poisson model equation, the mappings $a, b, c, \Lambda$ and the right-hand sides $\ell_{Q}$ and $\ell_{V}$ from the definitions of the operator $\mathscr{A}$ and the right-hand side $\ell$ in (2.1) read, for all $p, q \in Q$ and $u, v \in V$,

$$
\begin{aligned}
a(p, q) & :=\int_{\Omega} p \cdot q d x, \\
b(q, u) & :=\int_{\Omega} q \cdot \nabla u d x, \\
c(u, v) & :=0, \\
\Lambda u & :=\nabla u, \\
\ell_{Q}(q) & :=0, \\
\ell_{V}(v) & :=\int_{\Omega} f v d x .
\end{aligned}
$$

Some function $u \in V$ solves the weak form of the mixed Poisson equation if and only if

$$
\begin{array}{lc}
\forall q \in Q & a(p, q)-b(q, u)=\int_{\Omega}(p-\nabla u) \cdot q d x \stackrel{!}{=} 0=\ell_{Q}(q), \\
\forall v \in V & b(p, v)=\int_{\Omega} p \cdot \nabla v d x=-\int_{\Omega} \operatorname{div} p v d x \stackrel{!}{=} \int_{\Omega} f v d x=\ell_{V}(v) .
\end{array}
$$

Hence, problem (2.3) is recast as: Seek $(p, u) \in Q \times V$ with $\mathscr{A}(p, u)=\ell_{Q}+\ell_{V}$.

Remark 2.1. The operator $\mathscr{A}$ belonging to the Poisson problem is bounded, linear and bijective: An inf-sup property can be shown immediately because for any $(p, u) \in Q \times V$, with $(q, v):=(p-\nabla u, 2 u) \in Q \times V$ and the $H^{1}$ seminorm on $V=H_{0}^{1}(\Omega)$, it holds

$$
\begin{aligned}
1 / 5\|(p, u)\|_{Q \times V}\|(q, v)\|_{Q \times V} & \leq 1 / 5\left(\|p\|_{Q}+\|u\|_{V}\right)\left(\|p\|_{Q}+3\|u\|_{V}\right) \\
& \leq\|p\|_{Q}^{2}+\|u\|_{V}^{2}=(\mathscr{A}(p, u))(q, v) .
\end{aligned}
$$

Thus, the (generalized) Lax-Milgram lemma yields bijectivity of AA, cf. [Bra07, BS94].

\subsection{Stokes Problem}

Stationary incompressible fluid flow in a two- or three-dimensional domain can be modeled by the non-symmetric Stokes equations

$$
\begin{aligned}
-\Delta u+\nabla p=f & & \text { in } \Omega, \\
\operatorname{div} u=0 & & \text { in } \Omega, \\
u=0 & & \text { on } \partial \Omega
\end{aligned}
$$

for a velocity field $u$ and a pressure $p$ on a domain $\Omega \subset \mathbb{R}^{n}(n \in\{2,3\})$. The equations are naturally stated in a mixed form with a vector valued solution $u \in V:=H_{0}^{1}\left(\Omega ; \mathbb{R}^{n}\right)$ and with the pressure $p \in Q:=L_{0}^{2}(\Omega)$. In the symmetric formulation of the Stokes equations, one uses the symmetric part of the gradient of a vector valued function $u$ written as

$$
\varepsilon(u):=\frac{1}{2}\left(\nabla u+\nabla u^{T}\right) \in L^{2}\left(\Omega ; \mathbb{R}_{\mathrm{sym}}^{n \times n}\right) .
$$


The symmetric Stokes problem with viscosity parameter $\mu>0$ reads: Find $u$ and $p$ with

$$
\begin{array}{rlrl}
-\operatorname{div} \mu \varepsilon(u)+\nabla p & =f & & \text { in } \Omega, \\
\operatorname{div} u=0 & & \text { in } \Omega, \\
u=0 & & \text { on } \partial \Omega .
\end{array}
$$

The two formulations are equivalent in case $\mu=1$ assuming homogeneous boundary conditions for the velocity on the entire boundary $\partial \Omega$, cf. [Bra07, BF91].

The corresponding mappings read

$$
\begin{aligned}
a(p, q) & :=-\int_{\Omega} p q d x, \\
b(q, v) & :=-\int_{\Omega} q \operatorname{div} v d x, \\
c_{\text {sym }}(u, v) & :=\int_{\Omega} 2 \mu \varepsilon(u): \varepsilon(v) d x, \\
c_{\text {asym }}(u, v) & :=\int_{\Omega} \nabla u: \nabla v d x, \\
\Lambda u & :=\operatorname{div} u, \\
\ell_{Q}(p, q) & :=-\int_{\Omega} p q d x, \\
\ell_{V}(v) & :=\int_{\Omega} f \cdot v d x .
\end{aligned}
$$

Note that only for formal reasons $\ell_{Q}$ is a bilinear form which depends on the solution $p$ as well as on the test function $q$. It cancels out in the mixed formulation with $a(p, q)$. In the variational form, the symmetric Stokes equation reads: Given $f \in L^{2}\left(\Omega ; \mathbb{R}^{n}\right)$, seek $(u, p) \in V \times Q$ such that

$$
\begin{aligned}
& \forall q \in Q-b(q, u)=\int_{\Omega} q \operatorname{div} u d x \stackrel{!}{=} 0, \\
& \forall v \in V \quad b(p, v)+c_{\text {sym }}(u, v)=\int_{\Omega} 2 \mu \varepsilon(u): \varepsilon(v) d x-\int_{\Omega} p \operatorname{div} v d x \stackrel{!}{=} \int_{\Omega} f \cdot v d x .
\end{aligned}
$$

The variational unsymmetric Stokes equations are identical with $c_{\text {asym }}$ instead of $c_{\text {sym }}$.

Remark 2.2. The Stokes equations (2.4) exhibit a unique solution ( $p, u)$, cf. [Bra07, BS94, CH07, Car05].

\subsection{Lamé Problem}

In linear elasticity theory, the displacement $u$ and the symmetric stress tensor $\sigma$ of a body $\Omega$ under the influence of applied forces $f$ satisfy the Navier-Lamé equations. The linear stress-strain relation $\mathbb{C}: \mathbb{R}^{n \times n} \rightarrow \mathbb{R}^{n \times n}$ reads

$$
\mathbb{C} E:=\lambda \operatorname{tr}(E) \mathbb{1}+2 \mu E \text { for strain matrices } E \in \mathbb{R}^{n \times n}
$$

with Lamé parameters $\lambda, \mu>0$. The inverse relation reads

$$
\mathbb{C}^{-1} \sigma=1 /(2 \mu) \sigma-\lambda /(2 \mu(n \lambda-2 \mu)) \operatorname{tr}(\sigma) \mathbb{1} \text { for stress matrices } \sigma \in \mathbb{R}^{n \times n} .
$$


In the continuous model with the stress-strain relation $\sigma=\mathbb{C} \varepsilon(u)$, the resulting model problem reads: Given $f \in L^{2}\left(\Omega ; \mathbb{R}^{n}\right)$, find $u \in H_{0}^{1}\left(\Omega ; \mathbb{R}^{n}\right) \cap H^{2}\left(\Omega ; \mathbb{R}^{n}\right)$ such that

$$
f+\operatorname{div} \mathbb{C} \varepsilon(u)=0 \quad \text { in } \Omega .
$$

To employ the unified theory for residuals with respect to discrete approximations of the solution, let $V:=H_{0}^{1}\left(\Omega ; \mathbb{R}^{n}\right), Q:=L^{2}\left(\Omega ; \mathbb{R}_{\text {sym }}^{n \times n}\right)$ and set, for $\sigma, \tau \in Q$ and for $u, v \in V$,

$$
\begin{aligned}
a(\sigma, \tau) & :=\int_{\Omega}\left(\mathbb{C}^{-1} \sigma\right): \tau d x, \\
b(\tau, u) & :=\int_{\Omega} \tau: \varepsilon(u) d x, \\
c(u, v) & :=0 \\
\Lambda(u) & :=\mathbb{C} \varepsilon(u), \\
\ell_{V}(v) & :=\int_{\Omega} f \cdot v d x, \\
\ell_{Q}(\tau) & :=0 .
\end{aligned}
$$

A pair of functions $\sigma \in Q$ and $u \in V$ solves the mixed formulation of the Lamé problem if

$$
\begin{array}{ll}
\forall \tau \in Q & a(\sigma, \tau)-b(\tau, u)=\int_{\Omega}\left(\mathbb{C}^{-1} \sigma-\varepsilon(u)\right): \tau d x \stackrel{!}{=} 0 \\
\forall v \in V \quad b(\sigma, v)=\int_{\Omega} \sigma: \varepsilon(v) d x=-\int_{\Omega} \operatorname{div} \sigma \cdot v d x \stackrel{!}{=} \int_{\Omega} f \cdot v d x=\ell_{V}(v) .
\end{array}
$$

Remark 2.3. The elliptic PDE (2.5) has a unique solution $u$, see [Cia88]. The operator $\mathscr{A}:(Q \times V) \rightarrow(Q \times V)^{*}$ from (2.1) is linear, bounded and bijective, and the operator norms of $\mathscr{A}$ and $\mathscr{A}^{-1}$ are $\lambda$-independent, cf. [BCR04].

Remark 2.4. The method which is used to solve the problem depends on the representation of the problem. In Section 8, some mixed methods vary the spaces $Q$ and $V$, and the mappings $\mathscr{A}$ and $\ell$.

\subsection{Eddy Current Problem}

We consider the semi-discrete eddy current equations [Mon03]

$$
\begin{array}{rlrl}
\operatorname{curl} \mu^{-1} \operatorname{curl} u+\sigma u & =f & & \text { in } \Omega, \\
u \wedge v=0 & & \text { on } \partial \Omega
\end{array}
$$

in a bounded domain $\Omega \subset \mathbb{R}^{3}$ with data $f \in L^{2}\left(\Omega ; \mathbb{R}^{3}\right)$ and parameters $\mu, \sigma>0$. The variational form of (2.6) reads: Seek $u \in H_{0}(\operatorname{curl}, \Omega)$ such that

$$
\int_{\Omega}\left(\mu^{-1} \operatorname{curl} u \cdot \operatorname{curl} v+\sigma u \cdot v\right) d x=\int_{\Omega} f \cdot v d x \quad \text { for all } v \in H_{0}(\operatorname{curl}, \Omega) .
$$


The mixed formulation of (2.6) reads

$$
\begin{array}{ll}
\mu p-\operatorname{curl} u=0 & \text { in } \Omega, \\
\operatorname{curl} p+\sigma u=f & \text { in } \Omega .
\end{array}
$$

In accordance with our unified notation from Section 2.1 we set $V:=H_{0}(\operatorname{curl}, \Omega)$, $Q:=L^{2}\left(\Omega ; \mathbb{R}^{3}\right)$ and, for all $p, q \in Q$ and $u, v \in V$,

$$
\begin{aligned}
a(p, q) & :=\int_{\Omega} \mu p \cdot q d x, \\
b(q, u) & :=\int_{\Omega} \operatorname{curl} u \cdot q d x, \\
c(u, v) & :=\int_{\Omega} \sigma u \cdot v d x, \\
\Lambda(u) & :=\mu^{-1} \operatorname{curl} u, \\
\ell_{Q}(q) & :=0, \\
\ell_{V}(v) & :=\int_{\Omega} f \cdot v d x .
\end{aligned}
$$

The associated mixed formulation reads: Seek $(p, u) \in Q \times V$ such that

$$
\begin{array}{ll}
\forall q \in Q & a(p, q)-b(q, u)=\int_{\Omega}(\mu p-\operatorname{curl} u) \cdot q d x \stackrel{!}{=} 0 \\
\forall v \in V & b(p, v)+c(u, v)=\int_{\Omega} \operatorname{curl} v \cdot p+\sigma u \cdot v d x \stackrel{!}{=} \int_{\Omega} f \cdot v d x .
\end{array}
$$

Remark 2.5. The operator $\mathscr{A}:(Q \times V) \rightarrow(Q \times V)^{*}$ defined by (2.1) and (2.7) is continuous, linear and bijective, and thus allows a bounded inverse, $c f$. [CHO9].

\section{Errors and Residuals}

\subsection{Concept}

For the exact solution $(p, u) \in Q \times V$ of a well-posed problem (2.2) and some approximation $\left(p_{\ell}, u_{\ell}\right) \in Q_{\ell} \times V_{\ell}$, the error $e$ is defined as

$$
e:=(p, u)-\left(p_{\ell}, u_{\ell}\right)=\left(p-p_{\ell}, u-u_{\ell}\right) .
$$

Note that both, $(p, u)$ and $\left(p_{\ell}, u_{\ell}\right)$ map from $\Omega$ to some $\mathbb{R}$-vector-space. Thus, $e$ is well defined even if the approximation $p_{\ell}$ or $u_{\ell}$ does not belong to the space $Q$ or $V$, respectively. In general, it is of course not possible to calculate the exact error $e$, since the exact solution $(p, u)$ would read

$$
(p, u)=e+\left(p_{\ell}, u_{\ell}\right)
$$

For non-conforming discretisations, since $\left(p_{\ell}, u_{\ell}\right)$ does not need to belong to $Q \times V$, the error norm $\|e\|_{Q \times V}$ may not be well-defined and hence can not be estimated properly. The split $(p, u)$ is always done in such a way that $Q_{\ell} \subset Q$. However, it might be that $V_{\ell} \not \subset V$. In 
order to allow for an estimation of the error, $u_{\ell}$ thus may have to be further approximated by some $\tilde{u}_{\ell} \in V$. We will discuss the strategy to find an appropriate $\tilde{u}_{\ell}$ in Section 5.1 by means of the central Theorem 5.1.

For a solution $(p, u)$ and an approximation $\left(p_{\ell}, \tilde{u}_{\ell}\right) \in Q \times V$, the residual $\mathscr{R}$ es measures the image of the error $e$ under $\mathscr{A}$,

$$
\mathscr{R} e s:=\mathscr{A}(e)=\mathscr{A}(p, u)-\mathscr{A}\left(p_{\ell}, \tilde{u}_{\ell}\right) \in(Q \times V)^{*}
$$

With practical calculations in mind, one aims at an estimation of the residual (in its operator norm) by some a posteriori error estimators which are reliable and efficient. The continuity and boundedness of $\mathscr{A}$ is inherited to $\mathscr{R} e s$ and therefore, the error $\left(p-p_{\ell}, u-\tilde{u}_{\ell}\right)$ is equivalent to the residual,

$$
\|\mathscr{R} e s\|_{(Q \times V)^{*}} \approx\left\|\left(p-p_{\ell}, u-\tilde{u}_{\ell}\right)\right\|_{Q \times V} .
$$

Recall that $(p, u)$ solves $\mathscr{A}(p, u)=\ell_{Q}+\ell_{V}$. The identity (2.1) for $\mathscr{A}$ allows us to write the residual $\mathscr{R}$ es in terms of $a, b, c, \ell_{Q}$ and $\ell_{V}$ as

$$
\mathscr{R} e s(q, v)=\ell_{Q}(q)+\ell_{V}(v)-a\left(p_{\ell}, q\right)-b\left(p_{\ell}, v\right)+b\left(q, \tilde{u}_{\ell}\right)-c\left(\tilde{u}_{\ell}, v\right) .
$$

This is the sum of the partial residuals $\mathscr{R} e s_{Q} \in Q^{*}$ and $\mathscr{R} e s_{V} \in V^{*}$, namely

$$
\begin{aligned}
& \mathscr{R} e s_{Q}=\ell_{Q}-a\left(p_{\ell}, \cdot\right)+b\left(\cdot, \tilde{u}_{\ell}\right)=\ell_{Q}-a\left(p_{\ell}-\Lambda \tilde{u}_{\ell}, \cdot\right) \in Q^{*} \\
& \mathscr{R} e s_{V}=\ell_{V}-b\left(p_{\ell}, \cdot\right)-c\left(\tilde{u}_{\ell}, \cdot\right) \in V^{*}
\end{aligned}
$$

By equivalence of norms we observe that

$$
\left\|p-p_{\ell}\right\|_{Q}+\left\|u-\tilde{u}_{\ell}\right\|_{V} \approx\left\|\mathscr{R} e s_{Q}\right\|_{Q^{*}}+\left\|\mathscr{R} e s_{V}\right\|_{V^{*}}
$$

In case $\ell_{Q}=0$ and if $Q$ is a Hilbert space with scalar product $a(\cdot, \cdot)$, the fact that $\mathscr{R} e s_{Q} \in Q^{*}$ implies

$$
\left\|\mathscr{R} e s_{Q}\right\|_{Q^{*}}=\left\|p_{\ell}-\Lambda \tilde{u}_{\ell}\right\|_{Q}
$$

In Sections 6-9, error estimators for the different problems defined above will be derived. For this, a consistency residual $\mathscr{R} e s_{\text {cons }}$ and an equilibrium residual $\mathscr{R} e s_{\text {eq }}$ are defined and analysed in each case. Usually these coincide with the residuals $\mathscr{R} e s_{V}$ and $\mathscr{R} e s_{Q}$ with a few terms swapped to either of the residuals $\mathscr{R} e s_{\text {cons }}$ or $\mathscr{R} e s_{\text {eq }}$ whenever it seems more natural (cf. Sections 3.3-3.6 below).

Remark 3.1. This paper does neither aim at a convergence analysis nor at quasi-optimality of adaptive FEM. We note that these issues have been initiated in [Dör96] and subsequently studied in [BDD04, Ste07] and [CKNSO8]. 


\subsection{Hilbert Space Case}

The following Theorem 3.1 gives a characterisation for $\mathscr{A}$ being a scalar product on $\mathscr{H}=Q \times V$. This is the condition for the result in Theorem 3.2 where we derive a perturbation result which is illustrative to consider although it is limited to certain applications only. In their mixed formulation, all applications in this paper have $b \neq 0$, which means, with the first part of Theorem 3.1 in mind, that Theorem 3.2 cannot be applied. However, since condition $b(\Lambda u, v)=b(\Lambda v, u)$ in the second part of Theorem 3.1 is always satisfied, Theorem 3.2 can be applied in all conforming non-mixed finite element methods in this paper.

Theorem 3.1. 1. A bilinear form $\mathscr{A}:(Q \times V) \times(Q \times V) \rightarrow \mathbb{R}$ defined as in (2.1) with bilinear forms $a, b$ and $c$ is symmetric if and only if the mappings $a$ and $c$ are symmetric and if it holds $b=0$.

2. A bilinear form $\mathscr{B}: V \times V \rightarrow \mathbb{R}$ defined by $\mathscr{B}(u, v)=\mathscr{A}(\Lambda u, u)(\Lambda v, v)$ with bilinear forms $a, b$ and $c$ is symmetric if and only if $a$ and $c$ are symmetric and if for all $u, v \in V$ it holds $b(\Lambda u, v)=b(\Lambda v, u)$.

Proof. If $\mathscr{A}$ is symmetric, then for $p=q=0 \in Q$ and for all $u, v \in V$ it holds

$$
c(u, v)=\mathscr{A}(p, u)(q, v)=\mathscr{A}(q, v)(p, u)=c(v, u),
$$

which means $c$ is symmetric. Furthermore, for all $p, q \in Q$ and $u=v=0 \in V$ it holds

$$
a(p, q)=\mathscr{A}(p, u)(q, v)=\mathscr{A}(q, v)(p, u)=a(q, p) .
$$

Thus, $a$ is symmetric. Using the fact that $\mathscr{A}, a, b$ and $c$ are bilinear, one easily realizes that for all $(p, u),(q, v) \in Q \times V$ it holds

$$
a(p, q)+b(p, v)-b(q, u)+c(u, v)=a(q, p)+b(q, u)-b(p, v)+c(v, u)
$$

Subtracting $a(p, q)=a(q, p)$ and $c(u, v)=c(v, u)$ yields

$$
b(p, v)=b(q, u) .
$$

To proof part 1 of the theorem, set for example $q=0$ to show for all $(p, v) \in Q \times V$ that $b(p, v)=0$ which implies $b=0$. To proof part 2, assume $u, v \in V$ and set $p=\Lambda u$ and $q=\Lambda v$ to see $b(\Lambda v, u)=b(\Lambda u, v)$. The implications in the other directions are obvious.

Given a right-hand side $\ell \in \mathscr{H}^{*}$ and its Riesz representation $u$, i.e., $\mathscr{A} u=\ell$ in $\mathscr{H}$, suppose that $u_{\ell}$ is an approximation for $u$. Define the error $e$ and the residual $\mathscr{R} e s$ as in (3.1)-(3.2) by

$$
e=u-u_{\ell} \text { and } \mathscr{R} e s=\mathscr{A}(e)=\ell-\mathscr{A} u_{\ell} .
$$


Theorem 3.2 (Error Approximation/Characterisation). Let $\mathscr{A}$ be the scalar-product in the Hilbert space $\mathscr{H}$ and define $\mathscr{R} e s=\mathscr{A}(e, \cdot)=\mathscr{A}(e)$ for any $e \in \mathscr{H}$. Then, for all $v \in \mathscr{H}$ with $\|v\|_{\mathscr{H}}=1$, it holds

$$
\frac{\|e\|_{\mathscr{H}}-\mathscr{R} e s(v)}{\|e\|_{\mathscr{H}}}=\frac{1}{2}\left\|v-\frac{e}{\|e\|_{\mathscr{H}}}\right\|_{\mathscr{H}}^{2} .
$$

Proof. Some elementary algebraic calculations yield

$$
\begin{aligned}
\frac{\|e\|_{\mathscr{H}}-\mathscr{R} e s(v)}{\|e\|_{\mathscr{H}}} & =1-\mathscr{A}\left(\frac{e}{\|e\|_{\mathscr{H}}}\right)(v) \\
& =\frac{1}{2} \mathscr{A}\left(\frac{e}{\|e\|_{\mathscr{H}}}\right)\left(\frac{e}{\|e\|_{\mathscr{H}}}\right)-\mathscr{A}\left(\frac{e}{\|e\|_{\mathscr{H}}}\right)(v)+\frac{1}{2} \mathscr{A}(v)(v) \\
& =\frac{1}{2}\left\|v-\frac{e}{\|e\|_{\mathscr{H}}}\right\|_{\mathscr{H}}^{2} \cdot
\end{aligned}
$$

Theorem 3.2 concerns the task to obtain a good approximation of $\|e\|_{\mathscr{H}}$ by the residual $\mathscr{R} e s(v)$ for some test function $v$. It reveals that the function $v$ needs to be close to $e /\|e\|_{\mathscr{H}}$. Hence, the evaluation of the operator norm $\|\mathscr{R} e s\|_{\mathscr{H}^{*}}$ is, in general, equivalent in terms of complexity to the evaluation of the exact solution $u$. Consequently, alternative approaches for the calculation of upper and lower bounds for $\|\mathscr{R} e s\|_{\mathscr{H}^{*}}$ are required and it appears advisable to compute good upper and lower bounds instead of the exact norm of the residual.

\subsection{Poisson Problem}

With the definitions of Section 2.2, the residuals of the Poisson problem (2.3) result from the residual representation formula (3.3). For $q \in Q$, the residual $\mathscr{R} e s_{Q}$ depends on the choice of $\tilde{u}_{\ell}$,

$$
\mathscr{R} e s_{Q}(q)=\ell_{Q}(q)-a\left(p_{\ell}-\Lambda \tilde{u}_{\ell}, q\right)=\int_{\Omega}\left(\nabla \tilde{u}_{\ell}-p_{\ell}\right) \cdot q d x .
$$

Since $L^{2}(\Omega)$ is isometrically isomorphic to its dual $L^{2}(\Omega)^{*}$, this yields the equality

$$
\left\|\mathscr{R} e s_{Q}\right\|_{Q^{*}}=\left\|p_{\ell}-\nabla \tilde{u}_{\ell}\right\|_{Q} .
$$

The minimisation with respect to $\tilde{u}_{\ell}$ leads to the consistency part of the residual as discussed in Section 5.1 below.

For $v \in V$, the equilibrium residual for the Poisson problem $\mathscr{R} e s_{V}$ can be written as

$$
\mathscr{R} e s_{V}(v)=\ell_{V}(v)-b\left(p_{\ell}, v\right)-c\left(\tilde{u}_{\ell}, v\right)=\int_{\Omega} f v d x-\int_{\Omega} \nabla v \cdot p_{\ell} d x .
$$

For some general subset $Q_{\ell} \subset L^{2}\left(\Omega ; \mathbb{R}^{n}\right)$, the divergence $\operatorname{div} p_{\ell}$ is merely understood as some distribution in $H^{-1}(\Omega)$. An integration by parts shows that $f+\operatorname{div} p_{\ell}$ is the Rieszrepresentative of $\mathscr{R} e s_{V}$ which we write as

$$
\left\|\mathscr{R} e s_{V}\right\|_{V^{*}}=\left\|f+\operatorname{div} p_{\ell}\right\|_{H^{-1}(\Omega)} .
$$




\subsection{Stokes Problem}

With an approximation $\left(p_{\ell}, \tilde{u}_{\ell}\right)$ of the exact solution $(p, u)$ of the Stokes equations (2.4), the calculation

$$
\mathscr{R} e s_{Q}(q)=\ell_{Q}\left(p_{\ell}, q\right)-a\left(p_{\ell}-\Lambda \tilde{u}_{\ell}, q\right)=-\int_{\Omega} q \operatorname{div} \tilde{u}_{\ell} d x
$$

yields the following part of the consistency residual,

$$
\left\|\mathscr{R} e s_{Q}\right\|_{Q^{*}}=\left\|\operatorname{div} \tilde{u}_{\ell}\right\|_{L^{2}(\Omega)} .
$$

For the residual $\mathscr{R} e s_{V}$ of the symmetric equations, it holds

$$
\begin{aligned}
{\mathscr{R} e s_{V}(v)} & =\ell_{V}(v)-b\left(p_{\ell}, v\right)-c_{\text {sym }}\left(\tilde{u}_{\ell}, v\right) \\
& =\int_{\Omega} f \cdot v d x-\int_{\Omega} 2 \mu \varepsilon\left(\tilde{u}_{\ell}\right): \varepsilon(v) d x+\int_{\Omega} p_{\ell} \operatorname{div} v d x \\
& =\int_{\Omega} f \cdot v d x-\int_{\Omega}\left(2 \mu \varepsilon\left(\tilde{u}_{\ell}\right)-p_{\ell} \mathbb{1}\right): \varepsilon(v) d x .
\end{aligned}
$$

Notice that the symmetry of the discrete stress tensor

$$
\sigma_{\ell}:=2 \mu \varepsilon\left(\tilde{u}_{\ell}\right)-p_{\ell} \mathbb{1}
$$

allows $\sigma_{\ell}: \varepsilon(v)=\sigma_{\ell}: \nabla v$. This suggests a split of the last term

$$
\int_{\Omega}\left(2 \mu \varepsilon\left(\tilde{u}_{\ell}\right)-p_{\ell} \mathbb{1}\right): \varepsilon(v) d x=\int_{\Omega} 2 \mu\left(\varepsilon\left(\tilde{u}_{\ell}\right)-\varepsilon_{\ell}\left(u_{\ell}\right)\right): \varepsilon(v) d x+\int_{\Omega} \sigma_{\ell}: \nabla v d x
$$

and yields

$\left\|\mathscr{R} e s_{V}(v)\right\| \approx 2 \mu\left\|\varepsilon_{\ell}\left(u_{\ell}\right)-\varepsilon\left(\tilde{u}_{\ell}\right)\right\|_{L^{2}\left(\Omega ; \mathbb{R}^{n \times n}\right)}\|\varepsilon(v)\|_{L^{2}\left(\Omega ; \mathbb{R}^{n \times n}\right)}+\left|\int_{\Omega} f \cdot v d x+\int_{\Omega} \sigma_{\ell}: \nabla v d x\right|$.

The first term $\left\|\varepsilon_{\ell}\left(u_{\ell}\right)-\varepsilon\left(\tilde{u}_{\ell}\right)\right\|_{L^{2}\left(\Omega ; \mathbb{R}^{n}\right)}$ may be treated with the methods for consistency error estimators in Section 5.1. The remaining two terms are treated as the equilibrium residual according to Section 5.2. The non-symmetric case can be analysed in an analogous way.

\subsection{Lamé Problem}

An approximation $\left(\sigma_{\ell}, \tilde{u}_{\ell}\right)$ of the true solution $(\sigma, u)$ of the Lamé equations (2.5) leads to

$$
\mathscr{R} e s_{Q}(\tau)=\ell_{Q}(\tau)-a\left(\sigma_{\ell}-\Lambda \tilde{u}_{\ell}, \tau\right)=\int_{\Omega}\left(\varepsilon\left(\tilde{u}_{\ell}\right)-\mathbb{C}^{-1} \sigma_{\ell}\right): \tau d x
$$


Hence, the dual norm of the consistency residual reads

$$
\left\|\mathscr{R} e s_{Q}\right\|_{Q^{*}}=\left\|\varepsilon\left(\tilde{u}_{\ell}\right)-\mathbb{C}^{-1} \sigma_{\ell}\right\|_{L^{2}\left(\Omega ; \mathbb{R}_{\text {sym }}^{n \times n}\right)} .
$$

For any $v \in V$, the resulting equilibrium residual in $V^{*}$ is given by

$$
\mathscr{R} e s_{V}(v)=\ell_{V}(v)-b\left(\sigma_{\ell}, v\right)-c\left(\tilde{u}_{\ell}, v\right)=\int_{\Omega} f \cdot v d x-\int_{\Omega} \varepsilon(v): \sigma_{\ell} d x .
$$

Note that for all symmetric matrices $E, F \in \mathbb{R}_{\text {sym }}^{n \times n}$ it holds

$\mathbb{C} E: F=(\lambda \operatorname{tr}(E) \mathbb{1}+2 \mu E): F=\lambda \operatorname{tr}(E) \mathbb{1}: F+2 \mu E: F=\lambda \operatorname{tr}(E) \operatorname{tr} F+2 \mu E=\mathbb{C} F: E$.

Hence, the differential operator $\Lambda$ is symmetric in the form $b$ for the non-mixed formulation (cf. Theorem 3.1.2) with

$$
b(\Lambda u, v)=\int_{\Omega} \mathbb{C} \varepsilon(u): \varepsilon(v) d x=\int_{\Omega} \mathbb{C} \varepsilon(v): \varepsilon(u) d x=b(\Lambda v, u) .
$$

\subsection{Eddy Current Problem}

With an approximation $\left(p_{\ell}, \tilde{u}_{\ell}\right)$ of the exact solution $(p, u)$ of the eddy current equations (2.6), for all $q \in Q$ the residual $\mathscr{R} e s_{Q}$ reads

$$
\mathscr{R} e s_{Q}(q)=\ell_{Q}(q)-a\left(p_{\ell}-\Lambda \tilde{u}_{\ell}, q\right)=\int_{\Omega}\left(\operatorname{curl} \tilde{u}_{\ell}-\mu p_{\ell}\right) \cdot q d x .
$$

For the dual norm of this consistency residual one obtains

$$
\left\|\mathscr{R} e s_{Q}\right\|_{Q^{*}}=\left\|\mu p_{\ell}-\operatorname{curl} \tilde{u}_{\ell}\right\|_{L^{2}\left(\Omega ; \mathbb{R}^{n}\right)} .
$$

The other part $\mathscr{R} e s_{V}$ reads for all $v \in V$,

$$
\mathscr{R} e s_{V}(v)=\ell_{V}(v)-b\left(p_{\ell}, v\right)-c\left(\tilde{u}_{\ell}, v\right)=\int_{\Omega}\left(f-\sigma \tilde{u}_{\ell}\right) \cdot v d x-\int_{\Omega} p_{\ell} \cdot \operatorname{curl} v d x .
$$

The involved analysis of this equilibrium type residual $\mathscr{R} e s_{V}$ will be studied in Section 9 below.

\section{Finite Element Spaces}

This section is devoted to the most common finite element spaces. After some preliminary notation in Section 4.1, they are introduced in Section 4.2. The next Section 4.3 defines (quasi-)interpolation operators mapping from $V \times Q$ to $V_{\ell} \times Q_{\ell}$ in a generic way for a wide range of applications. The section is completed by a formal reformulation of the conforming, non-conforming and mixed discrete problems in the unified notation (Section 4.4). 


\subsection{Notation}

The construction of finite element spaces is based on the discretisation of a Lipschitz domain $\Omega \subset \mathbb{R}^{n}(n=2,3)$ which is a bounded, simply connected set with piecewise affine boundary $\partial \Omega$. Let $\mathscr{T}_{\ell}$ be a set of simplices in $\Omega$, i.e. in $2 \mathrm{D}$, a set of closed triangles $T \subset \bar{\Omega}$ of positive area $|T|$ respectively in 3D, a set of closed tetrahedrons $T \subset \Omega$ with positive volume $|T|$. The set $\mathscr{T}_{\ell}$ is called triangulation of $\Omega$ if $\bigcup \mathscr{T}_{\ell}=\bar{\Omega}$ and if for simplices $T_{1}, T_{2} \in \mathscr{T}_{\ell}$, it holds $T_{1}=T_{2}$ or the intersection of $T_{1}$ and $T_{2}$ has zero volume, that is, $\left|T_{1} \cap T_{2}\right|=0$. We denote the set of all nodes of simplices in $\mathscr{T}_{\ell}$ with $\mathscr{N}_{\ell}$, the set of inner nodes with $\mathscr{K}_{\ell}=\mathscr{N}_{\ell} \cap \Omega$, the set of all edges with $\mathscr{E}_{\ell}$ and in the 3D case the set of faces of tetrahedrons in $\mathscr{T}_{\ell}$ with $\mathscr{F}_{\ell}$. Furthermore, for $\omega \subset \bar{\Omega}$ and $p \in \bar{\Omega}$ we abbreviate

$$
\begin{aligned}
\mathscr{N}_{\ell}(\omega) & :=\mathscr{N}_{\ell} \cap \omega ; \\
\mathscr{K}_{\ell}(\omega) & :=\mathscr{K}_{\ell} \cap \omega ; \\
\mathscr{E}_{\ell}(\omega) & :=\left\{E \in \mathscr{E}_{\ell} \mid E \subset \omega\right\} ; \\
\mathscr{F}_{\ell}(\omega) & :=\left\{F \in \mathscr{F}_{\ell} \mid F \subset \omega\right\} ; \\
\mathscr{T}_{\ell}(\omega) & :=\left\{T \in \mathscr{T}_{\ell} \mid \omega \subset T\right\} \quad \text { and } \mathscr{T}_{\ell}(p):=\mathscr{T}_{\ell}(\{p\})
\end{aligned}
$$

and write $\operatorname{mid}(\omega)$ for the center of gravity for elements, faces or edges $\omega$. A triangulation $\mathscr{T}_{\ell}$ is called regular if it holds

$$
T_{1} \cap T_{2} \in \mathscr{T}_{\ell} \cup \mathscr{E}_{\ell} \cup \mathscr{F}_{\ell} \cup \mathscr{N}_{\ell} \cup\{\emptyset\} \quad \text { for simplices } T_{1} \text { and } T_{2} \in \mathscr{T}_{\ell} .
$$

For a triangle (tetrahedron) $T \in \mathscr{T}_{\ell}$ with area (volume) $|T|$ we define its size $h_{T}:=|T|^{1 / n} \approx$ $\operatorname{diam}(T)$. All triangulations in this paper are shape-regular in the sense that the volume $|T|$ is equivalent to the diameter $h_{T}$ of each simplex $T$. The piecewise constant mapping $h_{\mathscr{T}}: \bar{\Omega} \rightarrow \mathbb{R}$ is defined in an $L^{2}$ sense via $\left.h_{\mathscr{T}}\right|_{T}:=h_{T}$ for all $T \in \mathscr{T}_{\ell}$. Accordingly, for any edge $E \in \mathscr{E}_{\ell}$ (or face $F \in \mathscr{F}_{\ell}$ ), we denote its size by $h_{E}:=|E|$ (or $h_{F}:=|F|^{1 / 2}$ ) while $v_{E}$ (or, in $3 \mathrm{D} v_{F}$ ) and $\tau_{E}$ are the unit normal and unit tangential vectors. The mapping $h_{\mathscr{E}}: \bigcup \mathscr{E}_{\ell} \rightarrow \mathbb{R}$ is defined by $\left.h_{\mathscr{E}}\right|_{E}:=h_{E}$ for all $E \in \mathscr{E}_{\ell}$.

Additionally, for $z \in \mathscr{N}_{\ell}, E \in \mathscr{E}_{\ell}, F \in \mathscr{F}_{\ell}$, and $T \in \mathscr{T}_{\ell}$ we refer to $\Omega_{z}, \Omega_{E}, \Omega_{F}$, and $\Omega_{T}$ as the patches

$$
\begin{aligned}
& \Omega_{z}:=\bigcup \mathscr{T}_{\ell}(z), \quad \Omega_{E}:=\bigcup \mathscr{T}_{\ell}(E), \\
& \Omega_{F}:=\bigcup \mathscr{T}_{\ell}(F), \quad \Omega_{T}:=\bigcup\left\{T^{\prime} \in \mathscr{T}_{\ell} \mid T^{\prime} \cap T \neq \emptyset\right\} .
\end{aligned}
$$

\subsection{Discrete Spaces and Basis Functions}

Problems considered in this paper employ the spaces $H^{1}\left(\Omega ; \mathbb{R}^{m}\right), H(\operatorname{curl}, \Omega), H(\operatorname{div}, \Omega)$, and $L^{2}\left(\Omega ; \mathbb{R}^{m}\right)$. With appropriate differential operators, these spaces form an exact sequence [AFW06, AFW10] which is shown in the next commuting diagram together with corresponding conforming discrete spaces. The respective quasi-interpolation operators $J_{\ell}^{N}, J_{\ell}^{E}, J_{\ell}^{F}, J_{\ell}^{T}$ are defined in Section 4.3, the discrete spaces in the bottom line are defined below. 


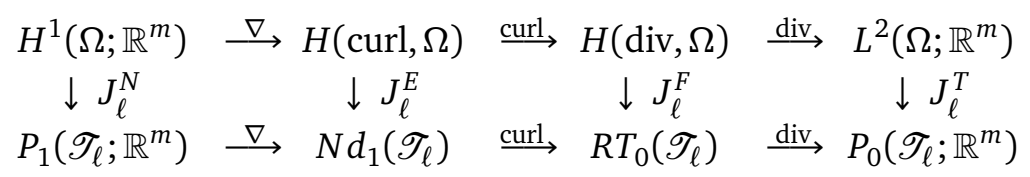

Conforming Discrete Spaces. $P_{k}\left(\omega ; \mathbb{R}^{m}\right)$ denotes the space of polynomials of total degree $\leq k$ which map from $\omega \subset \mathbb{R}^{n}$ to $\mathbb{R}^{m}$. For a triangulation $\mathscr{T}_{\ell}$, some common affine conforming discrete spaces are the Courant spaces $\left(P_{1}\right)$, Nédélec's first family of elements $\left(\mathrm{Nd}_{1}\right)$ and Raviart-Thomas spaces $\left(\mathrm{RT}_{0}\right)$, as shown in (4.1).

$$
\begin{aligned}
P_{1}\left(\mathscr{T}_{\ell} ; \mathbb{R}^{m}\right) & :=\left\{v \in L^{2}\left(\Omega ; \mathbb{R}^{m}\right)\left|\forall T \in \mathscr{T}_{\ell}, v\right|_{T} \in P_{1}\left(T ; \mathbb{R}^{m}\right)\right\} \cap H^{1}\left(\Omega ; \mathbb{R}^{m}\right), \\
P_{1,0}\left(\mathscr{T}_{\ell} ; \mathbb{R}^{m}\right) & :=P_{1}\left(\mathscr{T}_{\ell} ; \mathbb{R}^{m}\right) \cap H_{0}^{1}\left(\Omega ; \mathbb{R}^{m}\right), \\
\operatorname{Nd}_{1}(T) & :=\left\{v \mid \exists a, b \in \mathbb{R}^{3}, \forall x \in T, v(x):=a+b \wedge x\right\}, \\
\operatorname{Nd}_{1}\left(\mathscr{T}_{\ell}\right) & :=\left\{v \in V\left|\forall T \in \mathscr{T}_{\ell}, v\right|_{T} \in \operatorname{Nd}_{1}(T)\right\}, \\
\operatorname{Nd}_{1,0}\left(\mathscr{T}_{\ell}\right) & :=\left\{v \in V\left|\forall T \in \mathscr{T}_{\ell}, v\right|_{T} \in \operatorname{Nd}_{1}(T),\left.(v \wedge v)\right|_{\partial \Omega}=0\right\}, \\
\operatorname{RT}_{0}(T) & :=\left\{p \in L^{2}(\Omega) \mid p(x) \in P_{0}^{n}(T)+P_{0}(T) x, x \in \mathbb{R}^{n}\right\} \cap H\left(\operatorname{div}, \Omega ; \mathbb{R}^{n}\right), \\
\operatorname{RT}_{0}\left(\mathscr{T}_{\ell}\right) & :=\left\{p \in L^{2}(\Omega)\left|\forall T \in \mathscr{T}_{\ell}, p\right|_{T} \in \mathrm{RT}_{0}(T)\right\} .
\end{aligned}
$$

For the spaces defined above, we denote by $\left\{\varphi_{z} \mid z \in \mathscr{N}_{\ell}\right\},\left\{\varphi_{E} \mid E \in \mathscr{E}_{\ell}\right\},\left\{\varphi_{F} \mid F \in \mathscr{F}_{\ell}\right\}$, and $\left\{\varphi_{T} \mid T \in \mathscr{T}_{\ell}\right\}$ the nodal basis of $P_{1}\left(\mathscr{T}_{\ell}\right)$, the edge basis of $\operatorname{Nd}_{1}\left(\mathscr{T}_{\ell}\right)$, the face basis of $\mathrm{RT}_{0}\left(\mathscr{T}_{\ell}\right)$, and the element basis of $P_{0}\left(\mathscr{T}_{\ell}\right)$, respectively.

For convenience, we recall the construction of the common $P_{1}$-basis in more detail. The nodal basis functions $\varphi_{z}$ are defined for each node $z \in \mathscr{N}_{h}$ in two steps. First, the values at the nodes are given by

$$
\varphi_{z}(z)=1 \quad \text { and } \quad \varphi_{z}(x)=0 \quad \text { for any other node } x \in \mathscr{N}_{h} \backslash\{z\} .
$$

Then, given $\left.\varphi_{z}\right|_{\mathscr{N}_{h}}, \varphi_{z}$ is defined on each triangle $T=\operatorname{conv}\{A, B, C\}$ via affine interpolation. More precisely, $x \in T$ can be represented by $x=\alpha A+\beta B+\gamma C$ with convex coefficients $0 \leq \alpha, \beta, \gamma \leq 1$ where $\alpha+\beta+\gamma=1$. The evaluation of $\varphi_{z}$ at $x$ is obtained by

$$
\varphi_{z}(x)=\alpha \varphi_{z}(A)+\beta \varphi_{z}(B)+\gamma \varphi_{z}(C) .
$$

Since the triangulation is regular in the sense of Ciarlet [Cia02], this defines a globally continuous and piecewise affine function $\varphi_{z} \in H^{1}(\Omega)$. Figures 4.1 and 4.2 show the degrees of freedom and a $P_{1}$ basis function.

The construction of Nédélec basis functions is explained in Figures 4.3 and 4.4, RaviartThomas basis functions and the use of degrees of freedom are shown in Figures 4.5 and 4.6.

Non-Conforming Discrete Spaces. Crouzeix-Raviart spaces are common non-conform discrete spaces for $\Omega \subset \mathbb{R}^{2}$. We define

$$
\begin{aligned}
\mathrm{CR}_{1}\left(\mathscr{T}_{\ell} ; \mathbb{R}^{m}\right):= & \left\{v \in P_{1}\left(\mathscr{T}_{\ell} ; \mathbb{R}^{m}\right) \mid v \text { continuous in } \operatorname{mid}\left(\mathscr{E}_{\ell}\right)\right\}, \\
\mathrm{CR}_{1,0}\left(\mathscr{T}_{\ell} ; \mathbb{R}^{m}\right):= & \left\{v \in P_{1}\left(\mathscr{T}_{\ell} ; \mathbb{R}^{m}\right) \mid v \text { continuous in } \operatorname{mid}\left(\mathscr{E}_{\ell}\right),\right. \\
& \left.\forall E \in \mathscr{E}_{\ell} \text { with } E \subset \partial \Omega, v(\operatorname{mid}(E))=0\right\} .
\end{aligned}
$$



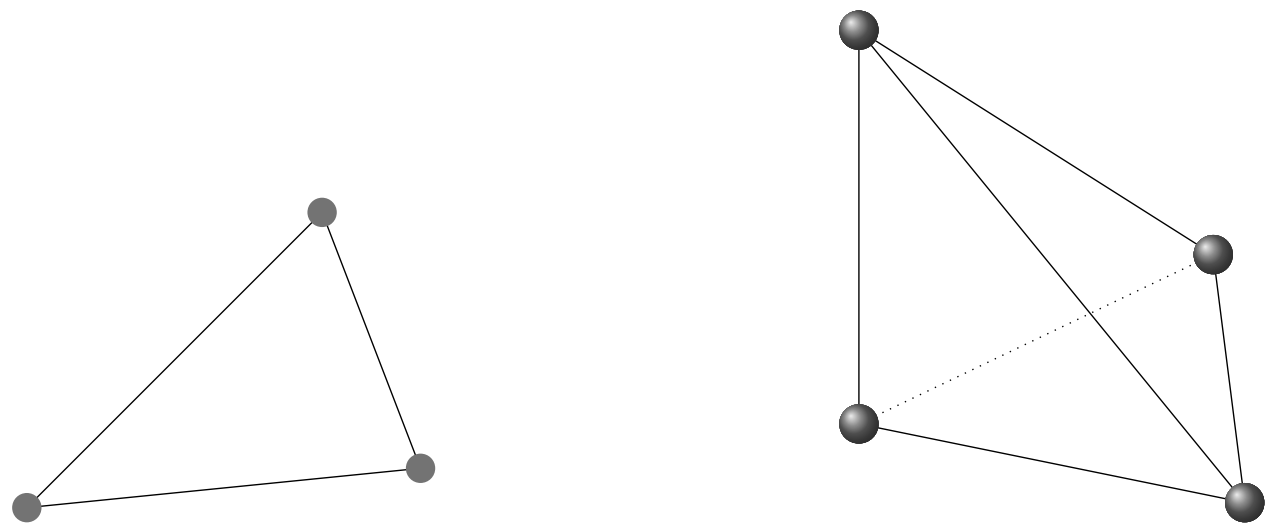

Figure 4.1: The $P_{1}$ functions in $\mathbb{R}^{2}$ and $\mathbb{R}^{3}$ have three resp. four degrees of feedom. The values of the function can be chosen for each node of the simplex - the resulting functions are $H^{1}$-conforming.

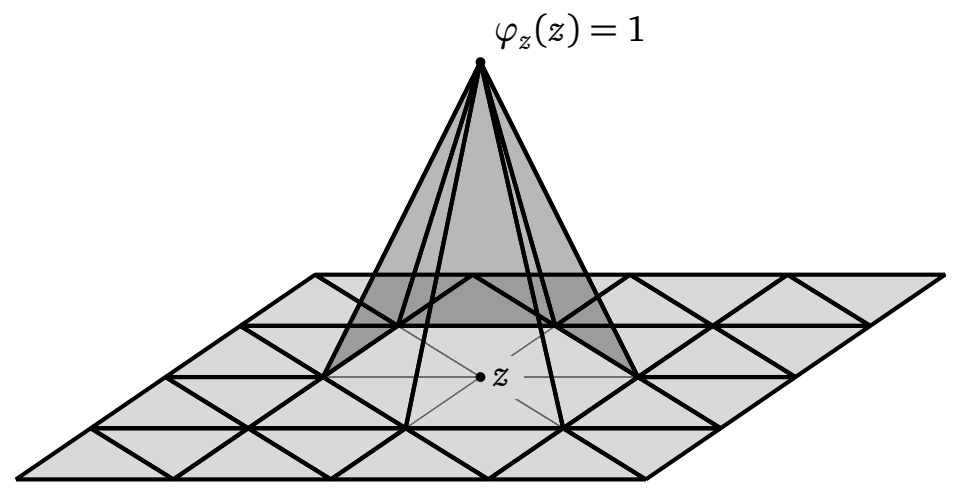

Figure 4.2: $P_{1}$ basis function $\varphi_{z}$ in $\mathbb{R}^{2}$ belonging to node $z$. The function is continuous and therefore $H^{1}$-conforming.

According to this, basis functions for $\mathrm{CR}_{1}\left(\mathscr{T}_{\ell} ; \mathbb{R}\right)$ are defined for edges $E$ of a triangle by

$$
\psi_{E} \in P_{1}\left(\mathscr{T}_{\ell}\right) \quad \text { s.t. } \quad \psi_{E}(\operatorname{mid}(F))=\delta_{E F} \quad \text { for } E, F \in \mathscr{E}_{\ell} .
$$

Figure 4.7 shows the use of the degrees of freedom and an example of a Crouzeix-Raviart basis function in $2 \mathrm{D}$.

Modified Basis Functions and Oscillations. Assume that each triangle $T \in \mathscr{T}_{\ell}$ has at least one node in $\mathscr{K}_{\ell} \subset \Omega$ and install a mapping $\zeta: \mathscr{N}_{\ell} \rightarrow \mathscr{K}_{\ell}$ such that $\left.\zeta\right|_{\mathscr{K}_{\ell}}=\left.i d\right|_{\mathscr{K}_{\ell}}$ and, for all $z \in \mathscr{N}_{\ell} \backslash \mathscr{K}_{\ell}, \zeta(z) \in \mathscr{K}_{\ell}\left(\mathscr{T}_{\ell}(z)\right)$ is a neighboring free node. As $\zeta$ might not be injective, the inverse mapping $\zeta^{-1}$ is set valued, $\zeta^{-1}(z):=\left\{y \in \mathscr{N}_{\ell}: \zeta(y)=z\right\}$.

Recall that $\left(\varphi_{z}\right)_{z \in \mathscr{N}_{\ell}}$ denotes the nodal basis of $P_{1}\left(\mathscr{T}_{\ell}\right) \cap C(\bar{\Omega})$. Then, for $z \in \mathscr{K}_{\ell}$, define functions $\psi_{z}$ via

$$
\psi_{z}:=\sum_{y \in \zeta^{-1}(z)} \varphi_{y} \in P_{1}\left(\mathscr{T}_{\ell}\right) \cap C(\Omega)
$$



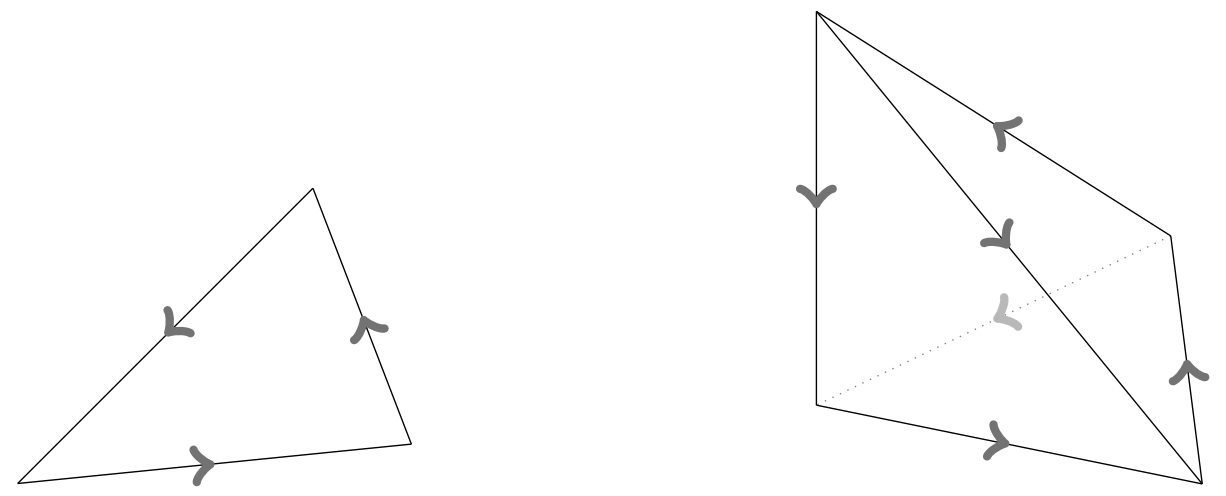

Figure 4.3: The Nédélec functions in $\mathbb{R}^{2}$ and $\mathbb{R}^{3}$ of the form $v\left(x_{1}, x_{2}\right)=\left(a_{1}-b x_{2}, a_{2}+b x_{1}\right)$ and $v(x)=a+b \wedge x$, respectively, have three resp. six degrees of feedom. For each edge, the tangential component can be chosen - the resulting functions are $H$ (curl)-conforming.

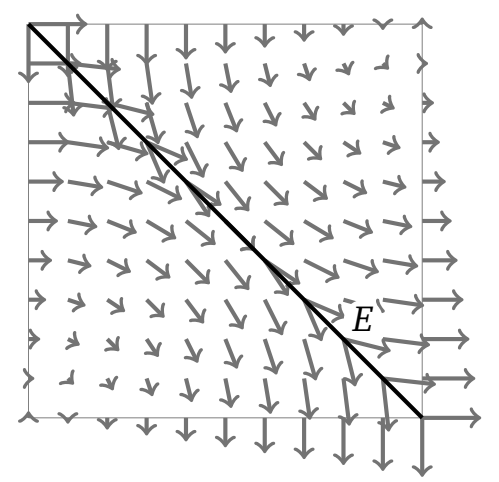

Figure 4.4: Nédélec basis function $\varphi_{E}$ in $\mathbb{R}^{2}$ belonging to the edge $E$. The tangential jumps over all edges are zero and $\varphi_{E}$ is therefore $H$ (curl)-conforming.

and the corresponding support sets $\operatorname{supp} \psi_{z}:=\left\{x \in \Omega: \psi_{z}(x)>0\right\}$.

Lemma 4.1. (a) The family $\left(\psi_{z}: z \in \mathscr{K}_{\ell}\right)$ is a Lipschitz-continuous partition of unity on $\Omega$,

$$
\sum_{z \in \mathscr{K}_{\ell}} \psi_{z}=1 \text { a.e. in } \Omega \text {, and for all } z \in \mathscr{K}_{\ell}, \quad 0 \leq \varphi_{z} \leq \psi_{z} \leq 1 \text {. }
$$

(b) From $\psi_{z} \neq \varphi_{z}$ it follows $\mathscr{E}_{\ell}\left(\mathscr{T}_{\ell}(z)\right) \cap \mathscr{E}_{\ell}(\partial \Omega) \neq \emptyset$ in $2 D$, i.e. at least one triangle with vertex $z$ has at least one edge on the boundary $\partial \Omega$ of the domain $\Omega$. The equivalent in $3 D$ is $\mathscr{F}_{\ell}\left(\mathscr{T}_{\ell}(z)\right) \cap \mathscr{F}_{\ell}(\partial \Omega) \neq \emptyset$.

(c) The supports supp $\psi_{z}$ have finite overlap, i.e.

$$
\max _{x \in \Omega, \ell \in \mathbb{N}}\left|\left\{z \in \mathscr{K}_{\ell}: x \in \operatorname{supp} \psi_{z}\right\}\right| \lesssim 1
$$

Proof. Details of the proof are left to the reader, cf. [Car97, Car99]. 

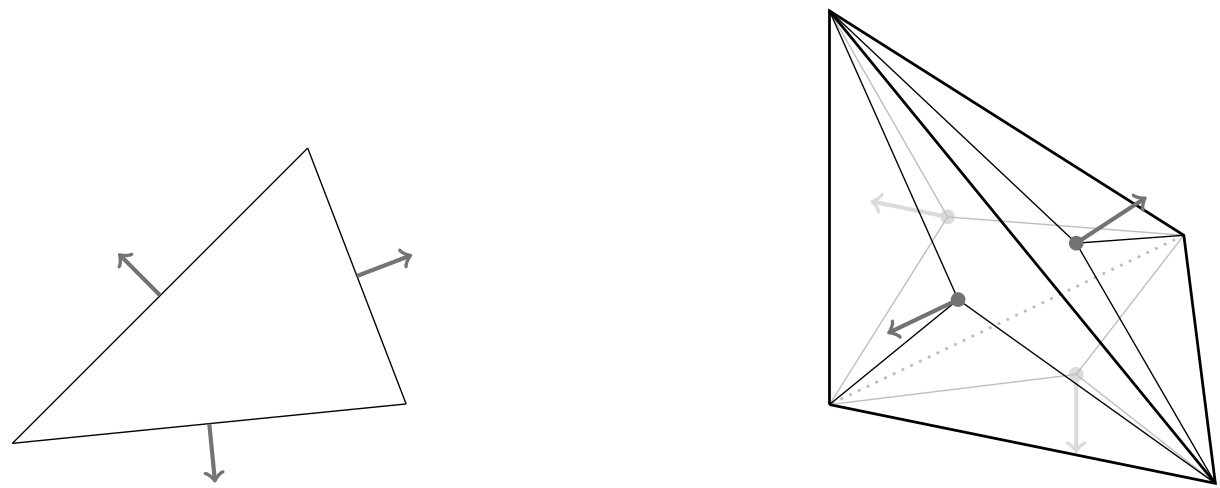

Figure 4.5: The Raviart-Thomas functions in $\mathbb{R}^{2}$ and $\mathbb{R}^{3}$ of the form $v\left(x_{1}, x_{2}\right)=\left(a_{1}+b x_{1}, a_{2}+b x_{2}\right)$, have three resp. four degrees of feedom. For each face, the normal component can be chosen - the resulting functions are $H$ (div)-conforming.

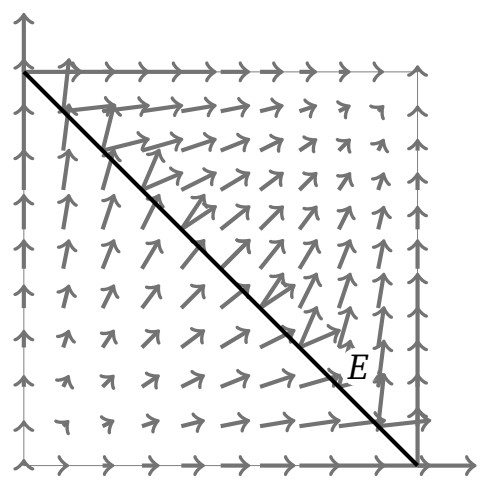

Figure 4.6: Raviart-Thomas basis function $\varphi_{E}$ in $\mathbb{R}^{2}$ belonging to the edge $E$. The normal jumps over all edges are zero and $\varphi_{E}$ is therefore $H$ (div)-conforming.

The following definition of oscillations and the Definition 4.1 below of the interpolation operator $J_{\ell}$ employ this partition of unity and allow the formulation of Theorem 5.2 which gives a posteriori estimators for equilibrium type residuals.

With the abbreviation $g_{\omega}:=|\omega|^{-1} \int_{\omega} g(x) d x \in \mathbb{R}^{m}$ for the integral mean of a function $g \in L^{2}\left(\omega ; \mathbb{R}^{m}\right)$ and any set $\mathscr{S}$ of measurable subsets $\omega$ of $\Omega$ of diameter $h_{\omega}:=\operatorname{diam}(\omega)$, the oscillation of $g$ on $\mathscr{S}$ is defined by

$$
\operatorname{osc}(g, \mathscr{S}):=\left(\sum_{\omega \in \mathscr{S}} h_{\omega}^{2}\left\|g-g_{\omega}\right\|_{L^{2}(S)}^{2}\right)^{1 / 2} .
$$

The data oscillation $\operatorname{osc}\left(g, \mathscr{T}_{\ell}\right)$ plays an important role. Additionally, we define oscillations subject to the set of free nodes $\mathscr{K}_{\ell}$ by

$$
\operatorname{Osc}\left(g, \mathscr{K}_{\ell}\right):=\operatorname{osc}\left(g,\left\{\operatorname{supp} \psi_{z}: z \in \mathscr{K}_{\ell}\right\}\right) .
$$

One can easily derive that $\operatorname{osc}\left(g, \mathscr{T}_{\ell}\right) \leq \operatorname{Osc}\left(g, \mathscr{K}_{\ell}\right)$. 

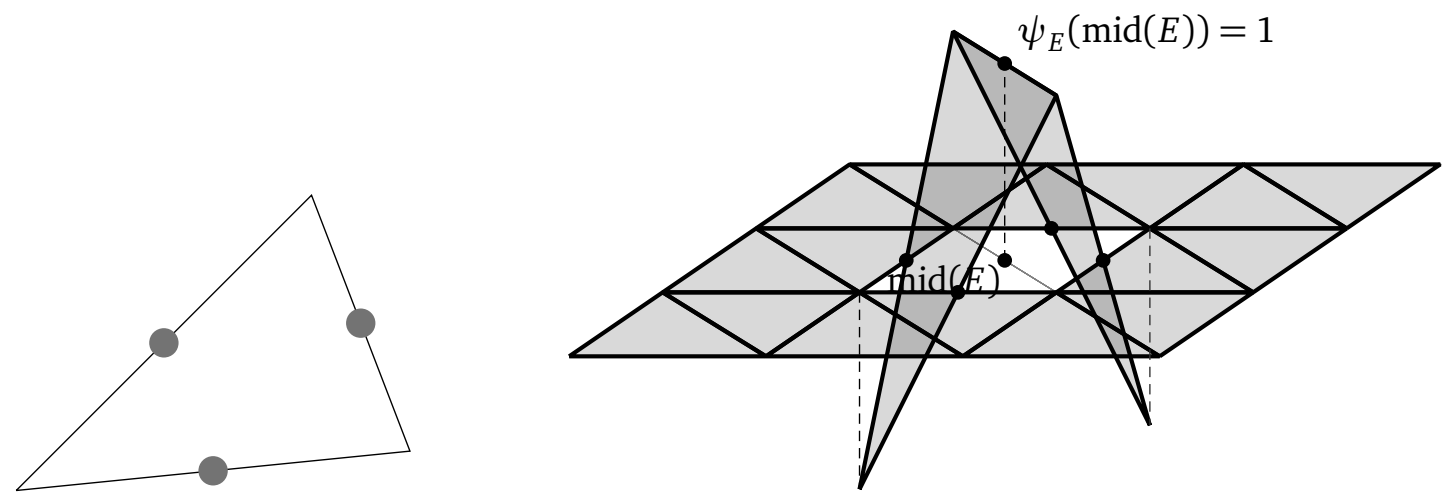

Figure 4.7: The $\mathrm{CR}_{1}$ functions in $\mathbb{R}^{2}$ have three degrees of feedom. The values of the function can be chosen for the midpoints of the edges/faces (left). The figure on the right shows the $\mathrm{CR}_{1}$ basis function $\psi_{E}$ belonging to edge $E$ (grey). The function is continuous in the midpoints of all edges.

\subsection{Interpolation Operators}

Quasi-interpolation operators for the sequence of spaces satisfying the commuting diagram property as depicted in (4.1) have been constructed in [Sch08] in the sense that

$$
\nabla J_{\ell}^{N}=J_{\ell}^{E} \nabla, \quad \operatorname{curl} J_{\ell}^{E}=J_{\ell}^{F} \operatorname{curl}, \quad \operatorname{div} J_{\ell}^{F}=J_{\ell}^{T} \operatorname{div} .
$$

The quasi-interpolation operators are defined as the compositions of the classical interpolation operators associated with the respective function spaces and appropriate smoothing operators. We emphasize that the incorporation of smoothing operators is mandatory, since the classical interpolation operators require continuous functions and, e.g., $H^{1}(\Omega) \subsetneq C(\bar{\Omega})$ for an open domain $\Omega$.

We recall that, for sufficiently smooth functions, the classical interpolation operators

$$
\begin{aligned}
I_{\ell}^{N}: H^{1}(\Omega) \rightarrow P_{1}\left(\Omega ; \mathbb{R}^{m}\right), & I_{\ell}^{E}: H(\operatorname{curl}, \Omega) \rightarrow \operatorname{Nd}_{1}\left(\mathscr{T}_{\ell}\right), \\
I_{\ell}^{F}: H(\operatorname{div}, \Omega) \rightarrow \operatorname{RT}_{0}\left(\mathscr{T}_{\ell}\right), & I_{\ell}^{T}: L^{2}(\Omega) \rightarrow P_{0}\left(\mathscr{T}_{\ell}\right)
\end{aligned}
$$


are given by

$$
\begin{aligned}
I_{\ell}^{N} v & :=\sum_{z \in \mathscr{N}_{\ell}} v(z) \varphi_{z} \\
I_{\ell}^{E} v & :=\sum_{E \in \mathscr{G}_{\ell}}\left(\int_{E} v \cdot \tau_{E} d s\right) \varphi_{E}, \\
I_{\ell}^{F} v & :=\sum_{F \in \mathscr{F}_{\ell}}\left(\int_{F} v \cdot v_{F} d \sigma\right) \varphi_{F}, \\
I_{\ell}^{T} v & :=\sum_{T \in \mathscr{T}_{\ell}}\left(\int_{T} v d x\right) \varphi_{T} .
\end{aligned}
$$

For $x \in \bar{\Omega}$ and $T \in \mathscr{T}_{\ell}$ we refer to $\left(\lambda_{z}^{T}(x)\right)_{z \in \mathscr{N}_{\ell}(T)}$ as the barycentric coordinates of $x$ in $T$, such that $x$ admits the representation $x=\sum_{z \in \mathscr{N}_{\ell}(T)} \lambda_{z}^{T}(x) z$ where all coordinates $\lambda_{z}^{T} \geq 0$ are non-negative and sum up to one, i.e. $\sum_{z \in \mathscr{N}(T)} \lambda_{z}^{T}=1$. For every node $z \in \mathscr{N}_{\ell}$, we choose $\omega_{z} \subset \Omega_{z}$ as a simply-connected domain with $z \in \omega_{z}$, e.g., $\omega_{z}:=B_{r}(z) \cap \Omega_{z}$ for some appropriately chosen $r>0$, where $B_{r}(z)$ stands for the ball with radius $r$ and center $z$.

For a triangle or tetrahedron $T \in \mathscr{T}_{\ell}, x \in \bar{\Omega}, y_{z} \in \omega_{z}, z \in \mathscr{N}_{\ell}(T)$ and $T^{\prime}:=\operatorname{conv}\left\{y_{z}: z \in\right.$ $\left.\mathscr{N}_{\ell}(T)\right\}$, we consider the transformation

$$
\hat{x}\left(x,\left(y_{z}\right)_{z \in \mathscr{N}_{\ell}(T)}\right):=\sum_{z \in \mathscr{N}_{\ell}(T)} \lambda_{z}^{T}(x) y_{z}
$$

see also Figure 4.8, and define the smoothing operators $S_{\ell}^{N}$ on $H^{1}(\Omega), S_{\ell}^{E}$ on $H(\operatorname{curl}, \Omega), S_{\ell}^{F}$ on $H(\operatorname{div}, \Omega)$, and $S_{\ell}^{T}$ on $L^{2}(\Omega)$ by means of

$$
\begin{aligned}
& \left(S_{\ell}^{N} v\right)(x):=\left(\prod_{z \in \mathscr{N}_{\ell}(T)}\left|\omega_{z}\right|\right)^{-1} \int_{\prod_{z \in \mathcal{N}_{\ell}(T)} \omega_{z}} v(\hat{x}) d\left(y_{z}\right)_{z \in \mathscr{N}_{\ell}(T)}\left(\frac{d \hat{x}}{d x}\right)^{T} v(\hat{x}) d\left(y_{z}\right)_{z \in \mathscr{N}_{\ell}(T)}, \\
& \left(S_{\ell}^{E} v\right)(x):=\left(\prod_{z \in \mathscr{N}_{\ell}(T)}\left|\omega_{z}\right|\right)^{-1} \int_{\prod_{z \in \mathcal{N}_{\ell}(T)} \omega_{z}}\left(\frac{d \hat{x}}{d x}\right)^{-T} v(\hat{x}) \operatorname{det}\left(\frac{d \hat{x}}{d x}\right) d\left(y_{z}\right)_{z \in \mathscr{N}_{\ell}(T)}, \\
& \left(S_{\ell}^{F} v\right)(x):=\left(\prod_{z \in \mathscr{N}_{\ell}(T)}\left|\omega_{z}\right|\right)^{-1} \int_{\prod_{z \in \mathcal{N}_{\ell}(T)} \omega_{z}} v(\hat{x}) \operatorname{det}\left(\frac{d \hat{x}}{d x}\right) d\left(y_{z}\right)_{z \in \mathscr{N}_{\ell}(T) .} \mid=\left(\prod_{z \in \mathscr{N}_{\ell}(T)}\left|\omega_{z}\right|\right)^{-1} \int_{\prod_{z \in \mathcal{N}_{\ell}(T)} \omega_{z}}\left(S_{\ell}^{T} v\right)(x):
\end{aligned}
$$




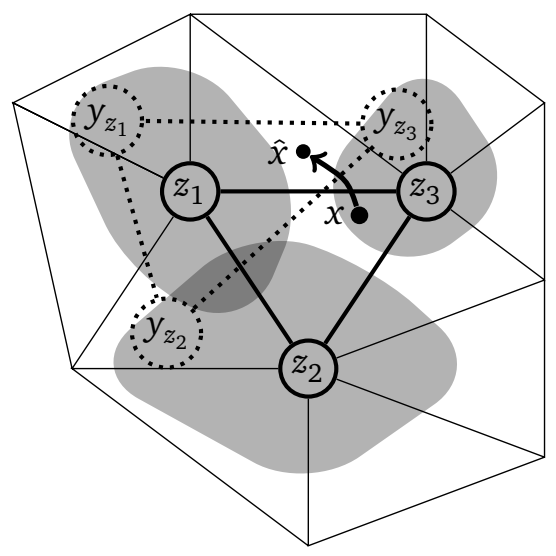

Figure 4.8: Illustration of the mapping $\hat{x}$ defined in equation (4.6). It maps a point $x$ in a triangle $T$ (printed bold) with nodes $z_{i}$ and fixed points $y_{z_{i}}$ in regions $\omega_{z_{i}}$ (grey shaded) around the triangle's nodes to a point $\hat{x}$ in the triangle $T^{\prime}=\operatorname{conv}\left\{y_{z}: z \in \mathscr{N}_{\ell}(T)\right\}$ (dotted).

In terms of the classical interpolation operators (4.5a)-(4.5d) and the smoothing operators (4.7a)-(4.7d), the quasi-interpolation operators are given by

$$
\begin{aligned}
J_{\ell}^{N}:=I_{\ell}^{N} \circ S_{\ell}^{N}, & J_{\ell}^{E}:=I_{\ell}^{E} \circ S_{\ell}^{E}, \\
J_{\ell}^{F}:=I_{\ell}^{F} \circ S_{\ell}^{F}, & J_{\ell}^{T}:=I_{\ell}^{T} \circ S_{\ell}^{T} .
\end{aligned}
$$

The commuting property (4.4) of diagram (4.1) follows from the corresponding properties of the classical interpolation operators and the smoothing operators (cf. Lemma 3 and Corollary 4 in [Sch08]). The quasi-interpolation operator $J_{\ell}^{N}$ shares the $H^{1}$-stability and local approximation properties of Clément's quasi-interpolation operator [Clé75, Car99]. The same applies to $J_{\ell}^{F}$ compared to the quasi-interpolation operator from [WH99]. As far as $J_{\ell}^{E}$ is concerned, we have the following result.

Theorem 4.1. Let $J_{\ell}^{E}: H_{0}(\operatorname{curl}, \Omega) \rightarrow \mathrm{Nd}_{1,0}\left(\mathscr{T}_{\ell}\right)$ be the quasi-interpolation operator given by (4.8a). Then, for every $v \in H_{0}(\operatorname{curl}, \Omega)$ there exist $\varphi \in H_{0}^{1}(\Omega)$ and $z \in H_{0}^{1}(\Omega)^{3}$ such that

$$
\begin{aligned}
v-J_{\ell}^{E} v & =\nabla \varphi+z, \\
h_{T}^{-1}\|\varphi\|_{L^{2}(T)}+\|\nabla \varphi\|_{L^{2}(T)} & \lesssim\|v\|_{L^{2}\left(\widetilde{\Omega}_{T}\right)}, \quad T \in \mathscr{T}_{\ell}, \\
h_{T}^{-1}\|z\|_{L^{2}(T)}+\|\nabla z\|_{L^{2}(T)} & \lesssim\|\operatorname{curl} v\|_{L^{2}\left(\widetilde{\Omega}_{T}\right)}, \quad T \in \mathscr{T}_{\ell},
\end{aligned}
$$

where the element patch $\widetilde{\Omega}_{T}$ is given by $\widetilde{\Omega}_{T}:=\bigcup\left\{T^{\prime} \in \mathscr{T}_{\ell} \mid T^{\prime} \cap \Omega_{T} \neq \emptyset\right\}$.

Proof. We refer to the proof of Theorem 1 in [Sch08].

Remark 4.1. A Scott-Zhang-type interpolation operator $\tilde{J}_{\ell}^{E}: H_{0}(\operatorname{curl}, \Omega) \cap H^{1}(\Omega)^{3} \rightarrow \operatorname{Nd}_{1,0}\left(\mathscr{T}_{\ell}\right)$ that admits similar local approximation properties as in Theorem 4.1 has been derived in [BHHWOO]. This result has been recently improved in $\left[\mathrm{ZCS}^{+} 10\right]$ without requiring extra regularity. 
For the proof of the reliability of the equilibrium estimator in Section 5.2 we make use of the following interpolation operator from [Car99].

Definition 4.1 (Weighted Interpolation Operator $J_{\ell}$ ). Given $g \in L^{2}(\Omega)$ set $J_{\ell} g \in P_{1,0}\left(\mathscr{T}_{\ell}\right)$ by

$$
J_{\ell} g:=\sum_{z \in \mathscr{K}_{\ell}}\left(\int_{\Omega} g \psi_{z} d x / \int_{\Omega} \varphi_{z} d x\right) \varphi_{z}
$$

to define an operator $J_{\ell}: L^{2}(\Omega) \rightarrow P_{1,0}\left(\mathscr{T}_{\ell}\right)$.

In addition, the operator $J_{\ell}$ fulfills some quasi-orthogonality and $H^{1}$-stability, see [Car99].

Theorem 4.2. For all $f \in L^{2}(\Omega)$ and $g \in H^{1}(\Omega)$, the interpolation operator of (4.10) and the set of free nodes $\mathscr{K}_{\ell}$ of a triangulation $\mathscr{T}_{\ell}$ of domain $\Omega$ it holds

$$
\int_{\Omega} f\left(g-J_{\ell} g\right) d x \lesssim \operatorname{Osc}\left(f, \mathscr{K}_{\ell}\right)\|\nabla g\|_{L^{2}(\Omega)} .
$$

Proof. For a free node $z \in \mathscr{K}_{\ell}$, set

$$
g_{z}:=\int_{\Omega} \psi_{z} g d x / \int_{\Omega} \varphi_{z} d x \quad \text { and } f_{\operatorname{supp} \psi_{z}}:=\left|\operatorname{supp} \psi_{z}\right|^{-1} \int_{\operatorname{supp} \psi_{z}} f d x .
$$

Since $\int_{\Omega}\left(\psi_{z} g-g_{z} \varphi_{z}\right) d x=0$ and $\sum_{z \in \mathscr{K}_{\ell}} \psi_{z}=1$, the Cauchy inequality yields

$$
\begin{aligned}
\int_{\Omega} f\left(g-J_{\ell} g\right) d x & =\sum_{z \in \mathscr{K}_{\ell}} \int_{\Omega} f\left(\psi_{z} g-g_{z} \varphi_{z}\right) d x \\
& =\sum_{z \in \mathscr{K}_{\ell}} \int_{\operatorname{supp} \psi_{z}}\left(f-f_{\text {supp } \psi_{z}}\right)\left(\psi_{z} g-g_{z} \varphi_{z}\right) d x \\
& \leq \operatorname{Osc}\left(f, \mathscr{K}_{\ell}\right)\left(\sum_{z \in \mathscr{K}_{\ell}} \operatorname{diam}\left(\operatorname{supp} \psi_{z}\right)^{-2}\left\|\psi_{z} g-g_{z} \varphi_{z}\right\|_{L^{2}\left(\operatorname{supp} \psi_{z}\right)}^{2}\right)^{1 / 2} .
\end{aligned}
$$

For every inner node $z \in \operatorname{supp} \psi_{z}$ the term $\operatorname{diam}\left(\operatorname{supp} \psi_{z}\right)^{-1}\left\|\psi_{z} g-g_{z} \varphi_{z}\right\|_{L^{2}\left(\operatorname{supp} \psi_{z}\right)}$ is bounded by $\|\nabla g\|_{L^{2}\left(\operatorname{supp} \psi_{z}\right)}$ and so (4.11) follows with Lemma 4.1.

\subsection{Discrete Problems}

Given the continuous problems $\mathscr{A}(p, u)=\ell_{Q}+\ell_{V}$ of (2.3), (2.4), (2.5), and (2.6) with differential operator $\Lambda$ and a regular triangulation $\mathscr{T}_{\ell}$ of the domain $\Omega$, the generic discrete problem reads: Find $\left(p_{\ell}, u_{\ell}\right) \in Q_{\ell} \times V_{\ell}$ such that

$$
\forall\left(q_{\ell}, v_{\ell}\right) \in Q_{\ell} \times V_{\ell} \quad \mathscr{A}_{\ell}\left(p_{\ell}, u_{\ell}\right)\left(q_{\ell}, v_{\ell}\right)=\ell_{Q}\left(q_{\ell}\right)+\ell_{V}\left(v_{\ell}\right)
$$


The modified discrete operator $\mathscr{A}_{\ell}$ depends on the class of the finite element method. In conforming methods, one uses $\mathscr{A}_{\ell}=\mathscr{A}$ and $p_{\ell}=\Lambda u_{\ell}$ and therefore one solves

$$
\mathscr{A}_{\ell}\left(\Lambda u_{\ell}, u_{\ell}\right)=a\left(\Lambda u_{\ell}, \Lambda \cdot\right)+c\left(u_{\ell}, \cdot\right)=\ell_{Q}+\ell_{V} .
$$

In non-conforming methods, although the differential operator $\Lambda$ is not defined on $V_{\ell}$, it induces some $\mathscr{T}_{\ell}$-piecewise operator

$$
\Lambda_{\ell}: V_{\ell} \rightarrow Q_{\ell} \quad \text { via }\left.\quad\left(\Lambda_{\ell} v_{\ell}\right)\right|_{T}=\left.\left.\Lambda\right|_{T} v_{\ell}\right|_{T} \text { for all } T \in \mathscr{T}_{\ell}
$$

With this operator $\Lambda_{\ell}$, set $p_{\ell}=\Lambda_{\ell} u_{\ell}$ and solve

$$
\mathscr{A}_{\ell}\left(\Lambda_{\ell} u_{\ell}, u_{\ell}\right)=a\left(\Lambda_{\ell} u_{\ell}, \Lambda_{\ell} \cdot\right)+c\left(u_{\ell}, \cdot\right)=\ell_{Q}+\ell_{V} .
$$

Mixed methods are formulated explicitly to provide a tuple $\left(p_{\ell}, u_{\ell}\right)$. In conforming mixed problems, one solves

$$
\mathscr{A}_{\ell}\left(p_{\ell}, u_{\ell}\right)=a\left(p_{\ell}, \cdot\right)+a\left(p_{\ell}, \Lambda \cdot\right)-a\left(\cdot, \Lambda u_{\ell}\right)+c\left(u_{\ell}, \cdot\right)=\ell_{Q}+\ell_{V} .
$$

In mixed non-conforming methods, $\Lambda$ is replaced by the piecewise analogue $\Lambda_{\ell}$ which yields the formulation

$$
\mathscr{A}_{\ell}\left(p_{\ell}, u_{\ell}\right)=a\left(p_{\ell}, \cdot\right)+a\left(p_{\ell}, \Lambda_{\ell} \cdot\right)-a\left(\cdot, \Lambda_{\ell} u_{\ell}\right)+c\left(u_{\ell}, \cdot\right)=\ell_{Q}+\ell_{V} .
$$

\section{Abstract Dual Norm Estimates}

This section is devoted to estimators for the consistency and equilibrium errors. In some cases it is convenient to keep the notation independent of the space dimension by definition of the set

$$
\mathscr{C}_{\ell}:= \begin{cases}\mathscr{E}_{\ell} & \text { for } n=2 \\ \mathscr{F}_{\ell} & \text { for } n=3\end{cases}
$$

Besides, we sometimes write "edges/faces" if we want to use edges in the 2D case and faces in 3D. We define the jump of a function $v$ on $C \in \mathscr{C}_{\ell}$ by $[v]_{C}:=\left.v\right|_{T_{1}}-\left.v\right|_{T_{2}}$ with $T_{1}$, $T_{2} \in \mathscr{T}_{\ell}(C), C=T_{1} \cap T_{2}$. The extension to all edges/faces $[v]_{\mathscr{C}}: \bigcup \mathscr{C}_{\ell} \rightarrow \mathbb{R}^{m}$ is given for all $C \in \mathscr{C}_{\ell}$ by $\left.[v]_{\mathscr{C}}\right|_{C}=[v]_{C}$. By $\langle v\rangle_{\mathscr{C}_{\ell}}$ we denote the mean value of test function $v$ in case of jumps at inner edges/faces and elsewhere the value of $v$.

\subsection{Consistency Error Estimates}

For a regular triangulation $\mathscr{T}_{\ell}$ of $\Omega \subset \mathbb{R}^{2}$ and functions $u_{\ell} \in V_{\ell}$ and $p_{\ell}=\nabla_{\ell} u_{\ell} \in Q_{\ell}$, our analysis of the consistency error is based on the minimum

$$
\min \left\{\left\|p_{\ell}-\nabla v\right\|_{L^{2}(\Omega)} \mid v \in V\right\} .
$$

In many situations, the following theorem provides several equivalent computable a posteriori error estimators. 
Theorem 5.1. On some domain $\Omega \subset \mathbb{R}^{2}$, for a Crouzeix-Raviart function $u_{\ell} \in \mathrm{CR}_{1}\left(\mathscr{T}_{\ell}\right)$ and with $V=H_{0}^{1}(\Omega)$ and $Q=L^{2}(\Omega)$ it holds

$$
\begin{aligned}
\min _{v \in V}\left\|\nabla_{\ell} u_{\ell}-\nabla v\right\|_{L^{2}(\Omega)} & \leq \min _{v_{\ell} \in P_{1,0}\left(\mathscr{T}_{\ell}\right)}\left\|\nabla_{\ell} u_{\ell}-\nabla v_{\ell}\right\|_{L^{2}(\Omega)} \\
& \lesssim \min _{v_{\ell} \in P_{1,0}\left(\mathscr{T}_{\ell}\right)}\left\|h_{\mathscr{T}}^{-1}\left(u_{\ell}-v_{\ell}\right)\right\|_{L^{2}(\Omega)} \\
& \leq\left\|h_{\mathscr{T}}^{-1}\left(u_{\ell}-A_{\mathscr{T}_{\ell}} u_{\ell}\right)\right\|_{L^{2}(\Omega)} \\
& \lesssim\left\|h_{\mathscr{E}}^{-1 / 2}\left[u_{\ell}\right]_{\mathscr{E}_{\ell}}\right\|_{L^{2}\left(\bigcup \mathscr{E}_{\ell}\right)} \\
& \lesssim\left\|h_{\mathscr{E}}^{1 / 2}\left[\nabla_{\ell} u_{\ell} \cdot \tau_{\mathscr{E}}\right]_{\mathscr{E}_{\ell}}\right\|_{L^{2}\left(\bigcup \mathscr{E}_{\ell}\right)} \\
& \lesssim \min _{v \in V}\left\|\nabla_{\ell} u_{\ell}-\nabla v\right\|_{L^{2}(\Omega)} .
\end{aligned}
$$

The averaging operator $A_{\mathscr{T}_{\ell}}: \mathrm{CR}_{1}\left(\mathscr{T}_{\ell}\right) \rightarrow P_{1}\left(\mathscr{T}_{\ell}\right)$ employed in the theorem is defined by

$$
A_{\mathscr{T}_{\ell}} u_{\ell}(z):=\left.\sum_{T \in \mathscr{T}_{\ell}(z)} u_{\ell}\right|_{T}(z) /\left|\mathscr{T}_{\ell}(z)\right| \quad \text { for all } z \in \mathscr{N}_{\ell}
$$

The following lemma is needed in the proof of Theorem 5.1.

Lemma 5.1. For any finite index set $J$ of length $|J| \in \mathbb{N}$ and a family $\left(a_{j} \mid j \in J\right)$ of real numbers with mean value $a:=\sum_{j \in J} a_{j} /|J|$, it follows that

$$
\sum_{j, k \in J}\left(a_{j}-a_{k}\right)^{2} \approx \sum_{j \in J}\left(a_{j}-a\right)^{2} .
$$

Proof. The calculation

$$
\begin{aligned}
|J|^{2} \sum_{j \in J}\left(a_{j}-a\right)^{2} & =\sum_{j \in J}\left(|J| a_{j}-\sum_{k \in J} a_{k}\right)^{2}=\sum_{j \in J}\left(\sum_{k \in J}\left(a_{j}-a_{k}\right)\right)^{2} \\
& \leq|J|^{2} \sum_{j \in J} \sum_{k \in J}\left(a_{j}-a_{k}\right)^{2}
\end{aligned}
$$

shows the inequality “ $\lesssim$ ". To prove the converse inequality, for $j, k \in J$ one observes that

$$
\left(a_{j}-a_{k}\right)^{2}=\left(\left(a_{j}-a\right)-\left(a_{k}-a\right)\right)^{2} \leq 2\left(a_{j}-a\right)^{2}+2\left(a_{k}-a\right)^{2} .
$$

The sum over all tuples $(j, k) \in J^{2}$ yields

$$
\sum_{j, k \in J}\left(a_{j}-a_{k}\right)^{2} \leq 2|J|^{2} \sum_{j \in J}\left(a_{j}-a\right)^{2} .
$$

Proof of Theorem 5.1. The first estimate (5.2a) holds due to the inclusion $P_{1,0}\left(\mathscr{T}_{\ell}\right) \subset V$, while inverse inequalities on every element domain $T \in \mathscr{T}_{\ell}$ yield (5.2b). To prove (5.2c), define $v_{\ell} \in P_{1,0}\left(\mathscr{T}_{\ell}\right)$ as $\mathscr{T}_{\ell}$-affine interpolation of the mean values

$$
v_{\ell}(z):=A_{\mathscr{T}_{\ell}} u_{\ell}(z)=\left.\sum_{T \in \mathscr{T}_{\ell}(z)} u_{\ell}\right|_{T}(z) /\left|\mathscr{T}_{\ell}(z)\right|
$$



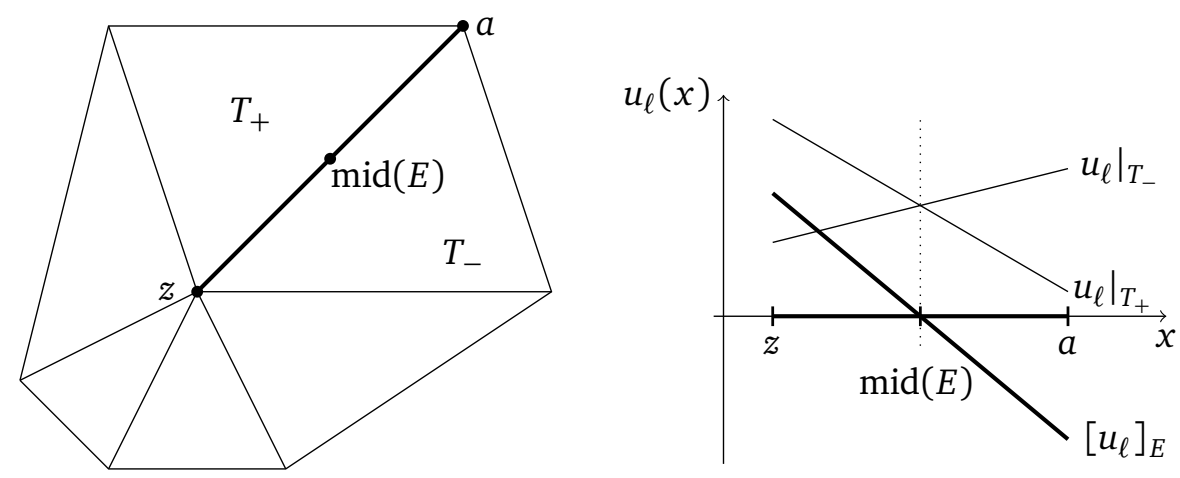

Figure 5.1: Illustration of the geometry and notation in the proof of (5.2d) of Theorem 5.1. To calculate the $L^{2}$-norm of the jump of the $\mathrm{CR}_{1}$-function $u_{\ell}$ along the edge $E=T_{+} \cap T_{-}$, merely $\left.u_{\ell}\right|_{T_{+}}$and $\left.u_{\ell}\right|_{T_{-}}$are evaluated in $z$.

at inner nodes $z \in \mathscr{K}_{\ell}$. (5.2c) follows from $A_{\mathscr{T}_{\ell}} u_{\ell} \in P_{1}\left(\mathscr{T}_{\ell}\right)$. Note that $v_{\ell}=\sum_{z \in \mathscr{K}_{\ell}} v_{\ell}(z) \varphi_{z} \in$ $V$ vanishes on the boundary. On each domain $T \in \mathscr{T}_{\ell},\left.\nabla_{\ell}\right|_{T}=\left.\nabla\right|_{T}$ and the argument in the $L^{2}$-norm is piecewise constant

$$
h_{T}^{-2}\left\|u_{\ell}-v_{\ell}\right\|_{L^{2}(T)}^{2} \approx|T| h_{T}^{-2} \sum_{z \in \mathscr{N}_{\ell}(T)}\left|u_{\ell}\right|_{T}(z)-\left.\left.v_{\ell}\right|_{T}(z)\right|^{2} .
$$

Notice $|T| h_{T}^{-2} \approx 1$. The sum over all triangles/tetrahedrons and a change of summation order yields

$$
\left\|h_{\mathscr{T}}^{-1}\left(u_{\ell}-v_{\ell}\right)\right\|_{L^{2}(\Omega)}^{2} \lesssim \sum_{z \in \mathscr{N}_{\ell}} \sum_{T \in \mathscr{T}_{\ell}(z)}\left|u_{\ell}\right|_{T}(z)-\left.\left.v_{\ell}\right|_{T}(z)\right|^{2} .
$$

For any $z \in \mathscr{N}_{\ell}$, Lemma 5.1 leads to

$$
\sum_{T \in \mathscr{T}_{\ell}(z)}\left|u_{\ell}\right|_{T}(z)-\left.\left.v_{\ell}\right|_{T}(z)\right|^{2} \approx \sum_{T, S \in \mathscr{T}_{\ell}(z)}\left|u_{\ell}\right|_{T}(z)-\left.\left.u_{\ell}\right|_{S}(z)\right|^{2} .
$$

Recall that $u_{\ell}$ is piecewise affine and continuous in the midpoints $\operatorname{mid}(E)$ of edges $E \in$ $\mathscr{E}_{\ell}(z)$. On the common edge $E$ of two adjacent triangles $T_{+}, T_{-} \in \mathscr{T}_{\ell}(z), E=T_{+} \cap T_{-}$, the $L^{2}(E)$ norm of the jump $\left[u_{\ell}\right]_{E}$ as shown in Figure 5.1 can therefore be calculated by

$$
\begin{aligned}
\left\|\left[u_{\ell}\right]_{\mathscr{E}_{\ell}}\right\|_{L^{2}(E)}^{2} & =\int_{0}^{h_{E}}\left(\frac{2\left(\left.u_{\ell}\right|_{T_{+}}(z)-\left.u_{\ell}\right|_{T_{-}}(z)\right)}{h_{E}} s-\left(\left.u_{\ell}\right|_{T_{+}}(z)-\left.u_{\ell}\right|_{T_{-}}(z)\right)\right)^{2} d s \\
& \approx h_{E}\left|u_{\ell}\right|_{T_{+}}(z)-\left.\left.u_{\ell}\right|_{T_{-}}(z)\right|^{2} .
\end{aligned}
$$

The combination of the aforementioned estimates results in

$$
\min _{v_{\ell} \in P_{1}\left(\mathscr{T}_{\ell}\right) \cap V}\left\|h_{\mathscr{T}}^{-1}\left(u_{\ell}-v_{\ell}\right)\right\|_{L^{2}(\Omega)} \lesssim\left\|h_{\mathscr{E}}^{-1 / 2}\left[u_{\ell}\right]_{\mathscr{E}_{\ell}}\right\|_{L^{2}\left(\bigcup \mathscr{E}_{\ell}\right)} .
$$

This proves (5.2d). 
For functions $v_{\ell} \in \mathrm{CR}_{1,0}$ for all edges $E \in \mathscr{E}_{\ell}$ it holds $\int_{E}\left[v_{\ell}\right]_{\mathscr{E}_{\ell}} d s=0$ and therefore the Poincaré inequality along $E$ yields the estimate (5.2e).

A standard argument with edge-bubble functions $b_{E}$ allows the proof of (5.2f), namely

$$
\left\|h_{\mathscr{E}}^{1 / 2}\left[\nabla_{\ell} u_{\ell} \cdot \tau_{\mathscr{E}}\right]_{\mathscr{E}_{\ell}}\right\|_{L^{2}\left(\bigcup \mathscr{E}_{\ell}\right)} \lesssim \min _{v \in V}\left\|\nabla_{\ell} u_{\ell}-\nabla v\right\|_{L^{2}(\Omega)}
$$

as follows, cf. [Ver96]. The quadratic edge-bubble function $b_{E}:=4 \varphi_{a} \varphi_{b}$ is defined for an edge $E:=\operatorname{conv}\{a, b\}$ with end-points $a, b \in \mathscr{N}_{\ell}$, subject to the affine nodal functions $\varphi_{a}, \varphi_{b} \in P_{1}\left(\mathscr{T}_{\ell}\right)$. The gradient $\nabla_{\ell} u_{\ell}$ is piecewise constant and on an edge $E \in \mathscr{E}_{\ell}$ it holds $\int_{E} b_{E} d s=\frac{2}{3}|E|$. Therefore,

$$
h_{E}^{1 / 2}\left\|\left[\nabla_{\ell} u_{\ell} \cdot \tau_{E}\right]_{\mathscr{E}_{\ell}}\right\|_{L^{2}(E)}=|E|\left|\left[\nabla_{\ell} u_{\ell} \cdot \tau_{E}\right]_{\mathscr{E}_{\ell}}\right| \approx\left|\int_{E} b_{E}\left[\nabla_{\ell} u_{\ell} \cdot \tau_{E}\right]_{\mathscr{E}_{\ell}} d s\right| .
$$

With $\nabla_{\ell} u_{\ell} \cdot \tau_{E}=\operatorname{Curl}_{\ell} u_{\ell} \cdot v_{E}$, an integration by parts leads to

$$
\int_{E} b_{E}\left[\nabla_{\ell} u_{\ell} \cdot \tau_{E}\right]_{\mathscr{E}_{\ell}} d s=\int_{\Omega_{E}} \nabla b_{E} \cdot \operatorname{Curl}_{\ell} u_{\ell} d x+\int_{\Omega_{E}} b_{E} \operatorname{div}_{\ell} \operatorname{Curl}_{\ell} u_{\ell} d x
$$

The last term vanishes because $u_{\ell} \in P_{1}\left(\mathscr{T}_{\ell}\right)$ is piecewise affine. Recall that $\Omega_{E}$ denotes the patch of the edge $E$. For all $v \in V$, the orthogonality $\nabla v \perp_{L^{2}(\Omega)} \operatorname{Curl}_{\ell} u_{\ell}$ leads to

$$
h_{E}^{1 / 2}\left\|\left[\nabla u_{\ell} \cdot \tau_{E}\right]_{\mathscr{G}_{\ell}}\right\|_{L^{2}(E)} \approx\left|\int_{\omega_{E}} \operatorname{Curl} b_{E}\left(\nabla_{\ell} u_{\ell}-\nabla v\right) d x\right| .
$$

The Cauchy inequality implies

$$
h_{E}^{1 / 2}\left\|\left[\nabla u_{\ell} \cdot \tau_{E}\right]_{\mathscr{E}_{\ell}}\right\|_{L^{2}(E)} \lesssim\left\|\operatorname{Curl} b_{E}\right\|_{L^{2}\left(\Omega_{E}\right)}\left\|\nabla_{\ell} u_{\ell}-\nabla v\right\|_{L^{2}\left(\Omega_{E}\right)} .
$$

The sum over all edges results in

$$
\begin{aligned}
\left(\sum_{E \in \mathscr{E}_{\ell}} h_{E}\left\|\left[\nabla_{\ell} u_{\ell} \cdot \tau_{E}\right]_{\mathscr{E}_{\ell}}\right\|_{L^{2}(E)}^{2}\right)^{1 / 2} & \lesssim\left(\sum_{E \in \mathscr{E}_{\ell}}\left\|\nabla_{\ell} u_{\ell}-\nabla v\right\|_{L^{2}\left(\Omega_{E}\right)}^{2}\right)^{1 / 2} \\
& \leq \sqrt{3}\left\|\nabla_{\ell} u_{\ell}-\nabla v\right\|_{L^{2}(\Omega)} .
\end{aligned}
$$

Since this is valid for all $v \in H_{0}^{1}(\Omega)$, the proof is finished.

\subsection{Explicit Equilibration Error Estimates}

This section is devoted to the estimation of residuals of the form

$$
R(v)=\int_{\Omega} R_{\mathscr{T}} \cdot v d x+\int_{\bigcup \mathscr{C}_{\ell}} R_{\mathscr{C}} \cdot\langle v\rangle_{\mathscr{C}_{\ell}} d s
$$


for arbitrary functions $R_{\mathscr{T}} \in L^{2}\left(\Omega ; \mathbb{R}^{m}\right)$ and $R_{\mathscr{C}} \in L^{2}\left(\bigcup \mathscr{C}_{\ell} ; \mathbb{R}^{m}\right)$ defined for a regular triangulation $\mathscr{T}_{\ell}$ of $\Omega$ with the associated set $\mathscr{C}_{\ell}$. Set $R_{T}:=\left.R_{\mathscr{T}}\right|_{T}$ for triangles/tetrahedrons $T \in \mathscr{T}_{\ell}$ and $R_{C}:=\left.R_{\mathscr{C}}\right|_{C}$ for $C \in \mathscr{C}_{\ell}$. Recall that $C$ can either be an edge (in 2D) or a face (in 3D) as defined in (5.1).

As mentioned before, the estimation of the dual norm of residuals structured like $R$ has been subject to intense research in the last decades, cf. [AO00, Ver96, BS01]. For any conforming discretisation considered here, it holds $V_{\ell}^{\mathrm{c}} \subset \operatorname{ker}\left(\mathscr{R} e s_{V}\right)$. Moreover, we require the following condition for the non-conforming discrete finite element space $V_{\ell}^{\text {nc }} \subset$ $H^{1}\left(\mathscr{T}_{\ell} ; \mathbb{R}^{m}\right):=\left\{v \in L^{2}\left(\Omega ; \mathbb{R}^{m}\right)\left|\forall T \in \mathscr{T}_{\ell}, v\right|_{T} \in H^{1}\left(\Omega ; \mathbb{R}^{m}\right)\right\}$ and its conforming counterpart $V_{\ell}^{\mathrm{c}} \subseteq V:=H_{0}^{1}\left(\Omega ; \mathbb{R}^{m}\right)$.

Assumption 5.1. There exists an operator $\Pi: V_{\ell}^{c} \rightarrow V_{\ell}^{n c}$ such that for all $v_{\ell} \in V_{\ell}^{c}$ and all $T \in \mathscr{T}_{\ell}$ it holds

$$
\left\|\nabla_{\ell}\left(\Pi v_{\ell}\right)\right\|_{L^{2}(\Omega)} \lesssim\left\|\nabla v_{\ell}\right\|_{L^{2}(\Omega)} \quad \text { and } \quad \int_{T} v_{\ell} d x=\int_{T} \Pi v_{\ell} d x
$$

Furthermore, for the discrete approximation $p_{\ell} \in L^{2}\left(\Omega ; \mathbb{R}^{m \times n}\right)$, it holds

$$
\int_{\Omega} p_{\ell}: \nabla v_{\ell} d x=\int_{\Omega} p_{\ell}: \nabla_{\ell}\left(\Pi v_{\ell}\right) d x
$$

Example 5.1. For $V_{\ell}^{n c}:=\mathrm{CR}_{1,0}\left(\mathscr{T}_{\ell}\right)$ and $V_{\ell}^{c}:=P_{1}\left(\mathscr{T}_{\ell}\right)$, the operator $\Pi$ is the identity.

Remark 5.1. Assumption 5.1 was introduced in [COHO7] called (H3) to generalize results for the equilibration residual estimation to a huge set of nonstandard finite elements. Assumptions (H1), (H2) from that reference are automatically fulfilled in all examples of the present paper.

Indeed, for each triangle $T \in \mathscr{T}_{\ell}$ and $v_{\ell} \in V_{\ell}^{c}:=P_{1}\left(\mathscr{T}_{\ell} ; \mathbb{R}^{m}\right), \int_{T}\left(v_{\ell}-\Pi v_{\ell}\right) d x=0$ by (5.4) and so Poincaré's inequality provides

$$
\left\|h_{T}^{-1}\left(v_{\ell}-\Pi v_{\ell}\right)\right\|_{L^{2}(T)} \lesssim\left\|\nabla v_{\ell}\right\|_{L^{2}(T)}+\left\|\nabla \Pi v_{\ell}\right\|_{L^{2}(T)} .
$$

The sum over all elements $T \in \mathscr{T}_{\ell}$ plus (5.4) implies

$$
\left\|h_{\mathscr{T}}^{-1}\left(v_{\ell}-\Pi v_{\ell}\right)\right\|_{L^{2}(\Omega)} \lesssim\left\|\nabla v_{\ell}\right\|_{L^{2}(\Omega)} .
$$

Theorem 5.2. Suppose the linear functional $R: V+V_{\ell}^{n c} \rightarrow \mathbb{R}$ satisfies $V_{\ell}^{n c} \subset \operatorname{ker} R$ for the non-conforming finite element space $V_{\ell}^{\text {nc }}$ and can be written in the form (5.3). Moreover, suppose there is an operator $\Pi$ according to Assumption 5.1 and consider the estimator

$$
\eta_{\ell}:=\left(\sum_{C \in \mathscr{C}_{\ell}} h_{C}\left\|R_{C}\right\|_{L^{2}(C)}^{2}\right)^{1 / 2}=\left\|h_{\mathscr{C}}^{1 / 2} R_{\mathscr{C}}\right\|_{L^{2}\left(\bigcup \mathscr{C}_{\ell}\right)}
$$

associated to some triangulation $\mathscr{T}_{\ell}$ with the set of free nodes $\mathscr{K}_{\ell}$. Then $\eta_{\ell}$ is a reliable and efficient estimator for $\|R\|_{V^{*}}$, i.e., it holds

$$
\begin{aligned}
\eta_{\ell} & \lesssim\|R\|_{V^{*}}+\operatorname{osc}\left(R_{\mathscr{T}}, \mathscr{T}_{\ell}\right)+\operatorname{osc}\left(R_{E}, \mathscr{E}_{\ell}\right), \\
\|R\|_{V^{*}} & \lesssim \eta_{\ell}+\operatorname{Osc}\left(R_{\mathscr{T}}, \mathscr{K}_{\ell}\right) .
\end{aligned}
$$


Proof. [of (5.6b)] Let $\Pi: V_{\ell}^{\mathrm{c}} \rightarrow V_{\ell}^{\mathrm{nc}}$ be the operator of Assumption 5.1 and recall the approximation operator $J_{\ell}$ from Definition 4.1. For any $v \in V$, the linearity of $R$ and $\Pi J_{\ell} v \in V_{\ell}^{\mathrm{nc}} \subset \operatorname{ker} R$ imply

$$
R(v)=R\left(v-\Pi J_{\ell} v\right)=\int_{\Omega} R_{\mathscr{T}} \cdot\left(v-\Pi J_{\ell} v\right) d x+\int_{\bigcup \mathscr{E}_{\ell}} R_{\mathscr{E}} \cdot\left\langle v-\Pi J_{\ell} v\right\rangle_{E} d s
$$

The volume term can be estimated with the Cauchy inequality, estimation (4.11) in Theorem 4.2, and with the oscillation (4.3),

$$
\begin{aligned}
& \int_{\Omega} R_{\mathscr{T}} \cdot\left(v-\Pi J_{\ell} v\right) d x \\
& =\sum_{T \in \mathscr{T}_{\ell}}\left(\int_{T} R_{T} \cdot\left(v-J_{\ell} v\right) d x+\int_{T}\left(R_{T}-\bar{R}_{T}\right) \cdot\left(J_{\ell} v-\Pi J_{\ell} v\right) d x\right) \\
& \lesssim \operatorname{Osc}\left(R_{\mathscr{T}}, \mathscr{K}_{\ell}\right)\|\nabla v\|_{L^{2}(\Omega)}+\operatorname{osc}\left(R_{\mathscr{T}}, \mathscr{T}_{\ell}\right)\left\|h_{\mathscr{T}}^{-1}\left(J_{\ell} v-\Pi J_{\ell} v\right)\right\|_{L^{2}(\Omega)} .
\end{aligned}
$$

With the estimate (5.5) and the stability of $J_{\ell}$, the last norm can be further bounded by

$$
\left\|h_{\mathscr{T}}^{-1}\left(J_{\ell} v-\Pi J_{\ell} v\right)\right\|_{L^{2}(\Omega)} \lesssim\left\|\nabla J_{\ell} v\right\|_{L^{2}(\Omega)} \lesssim\|\nabla v\|_{L^{2}(\Omega)} .
$$

Hence,

$$
\int_{\Omega} R_{\mathscr{T}} \cdot\left(v-\Pi J_{\ell} v\right) d x \lesssim \operatorname{Osc}\left(R_{\mathscr{T}}, \mathscr{K}_{\ell}\right)\|v\|_{H^{1}(\Omega)} .
$$

To analyze the edge/face terms, Cauchy's inequality leads to

$$
\int_{\bigcup \mathscr{C}_{\ell}} R_{\mathscr{C}} \cdot\left\langle v-\Pi J_{\ell} v\right\rangle_{\mathscr{C}_{\ell}} d s \leq \sum_{C \in \mathscr{C}_{\ell}}\left\|R_{C}\right\|_{L^{2}(C)}\left\|\left\langle v-\Pi J_{\ell} v\right\rangle_{C}\right\|_{L^{2}(C)}
$$

For each interior edge/face $C=T_{+} \cap T_{-} \in \mathscr{C}_{\ell}$ with $T_{+}, T_{-} \in \mathscr{T}_{\ell}$, the trace inequality shows

$$
\begin{aligned}
\left\|\left\langle v-\Pi J_{\ell} v\right\rangle_{C}\right\|_{L^{2}(C)} & \leq \frac{1}{2}\left\|\left.\left(v-\Pi J_{\ell} v\right)\right|_{T_{+}}\right\|_{L^{2}(C)}+\frac{1}{2}\left\|\left.\left(v-\Pi J_{\ell} v\right)\right|_{T_{-}}\right\|_{L^{2}(C)} \\
& \lesssim h_{C}^{1 / 2}\left\|\nabla\left(v-\Pi J_{\ell} v\right)\right\|_{L^{2}\left(\Omega_{C}\right)}+h_{C}^{-1 / 2}\left\|v-\Pi J_{\ell} v\right\|_{L^{2}\left(\Omega_{C}\right)} .
\end{aligned}
$$

On boundary edges/faces $C \in \partial \Omega \cap \partial T$, the norm

$$
\left\|\left\langle v-\Pi J_{\ell} v\right\rangle_{C}\right\|_{L^{2}(C)}=\left\|v-\Pi J_{\ell} v\right\|_{L^{2}(C)}
$$

is estimated by the same bound with $\Omega_{C}=\operatorname{int}(T)$. The sum over all edges/faces of $\mathscr{C}_{\ell}$ reads

$$
\int_{\bigcup \mathscr{C}_{\ell}} R_{\mathscr{C}} \cdot\left\langle v-\Pi J_{\ell} v\right\rangle_{\mathscr{C}_{\ell}} d s \lesssim\left\|h_{\mathscr{C}}^{1 / 2} R_{\mathscr{C}}\right\|_{L^{2}\left(\bigcup \mathscr{C}_{\ell}\right)}\left(\left\|\nabla\left(v-\Pi J_{\ell} v\right)\right\|_{L^{2}(\Omega)}+\left\|h_{\mathscr{T}}^{-1}\left(v-\Pi J_{\ell} v\right)\right\|_{L^{2}(\Omega)}\right) .
$$


The approximation and stability property of the last terms is controlled via the split

$$
v-\Pi J_{\ell} v=\left(v-J_{\ell} v\right)+\left(J_{\ell} v-\Pi J_{\ell} v\right) .
$$

First, Theorem 4.2 implies

$$
\left\|\nabla\left(v-J_{\ell} v\right)\right\|_{L^{2}(\Omega)}+\left\|h_{\mathscr{T}}^{-1}\left(v-J_{\ell} v\right)\right\|_{L^{2}(\Omega)} \lesssim\|\nabla v\|_{L^{2}(\Omega)} .
$$

Second, inequality (5.5) reveals

$$
\left\|\nabla\left(J_{\ell} v-\Pi J_{\ell} v\right)\right\|_{L^{2}(\Omega)}+\left\|h_{\mathscr{T}}^{-1}\left(J_{\ell} v-\Pi J_{\ell} v\right)\right\|_{L^{2}(\Omega)} \lesssim\|\nabla v\|_{L^{2}(\Omega)} .
$$

The combination of the two estimates plus (5.7) implies

$$
\left\|\nabla\left(v-\Pi J_{\ell} v\right)\right\|_{L^{2}(\Omega)}+\left\|h_{\mathscr{T}}^{-1}\left(v-\Pi J_{\ell} v\right)\right\|_{L^{2}(\Omega)} \lesssim\|\nabla v\|_{L^{2}(\Omega)} .
$$

Therefore,

$$
\|R\|_{V^{*}} \lesssim \operatorname{Osc}\left(R_{\mathscr{T}}, \mathscr{T}_{\ell}\right)+\left\|h_{\mathscr{C}}^{1 / 2} R_{\mathscr{C}}\right\|_{L^{2}\left(\bigcup \mathscr{C}_{\ell}\right)}
$$

Proof. [of (5.6a)] With $\Omega \subset \mathbb{R}^{m}$, for an edge or face $C=T_{+} \cap T_{-} \in \mathscr{C}_{\ell}$ and simplices $T_{+}, T_{-} \in \mathscr{T}_{\ell}$, recall that for the bubble functions

$$
b_{C}=\prod_{z \in \mathscr{N}(C)} \varphi_{z} \quad \text { and } \quad b_{T}=\prod_{z \in \mathscr{N}(T)} \varphi_{z}
$$

it holds for all $D \in \mathscr{C}_{\ell}$ and $D \neq C$

$$
\int_{D} b_{C} d s=0 \text { and } \int_{D} b_{T} d s=0
$$

For $C=T_{+} \cup T_{-} \in \mathscr{C}_{\ell}, T_{+}, T_{-} \in \mathscr{T}_{\ell}$, define the function

$$
v_{C}=\alpha_{\mathscr{C}} b_{C}+\alpha_{\mathscr{T}} b_{T_{+}}+\alpha_{\mathscr{T}} b_{T_{-}}
$$

and choose the two real numbers

$$
\alpha_{\mathscr{C}}=|C|\left(\int_{C} b_{C} d s\right)^{-1} \text { and } \alpha_{\mathscr{T}}=-\frac{|C| \int_{T_{+}} b_{C} d x}{\int_{C} b_{C} d s \int_{T} b_{T} d x} .
$$

In 2D we obtain $\alpha_{\mathscr{C}}=6$ and $\alpha_{\mathscr{T}}=-30$, in 3D a simple calculation reveals $\alpha_{\mathscr{C}}=120$ and $\alpha_{\mathscr{T}}=-840$. Then, for all $C, D \in \mathscr{C}_{\ell}$,

$$
\int_{D} v_{C} d s=|C| \delta_{C D} \quad \text { and, for all } T \in \mathscr{T}_{\ell}, \quad \int_{T} v_{C} d x=0 .
$$

Observe that

$$
h_{C}^{1 / 2}\left\|R_{C}\right\|_{L^{2}(C)} \leq h_{C}^{1 / 2}\left\|R_{C}-\bar{R}_{C}\right\|_{L^{2}(C)}+h_{C}^{1 / 2}\left\|\bar{R}_{C}\right\|_{L^{2}(C)}=\operatorname{osc}\left(R_{c}, C\right)+h_{C}^{1 / 2}\left\|\bar{R}_{C}\right\|_{L^{2}(C)}
$$


and set $\sigma:=\operatorname{sign}\left(\int_{C} \bar{R}_{C} d s\right)$ to obtain

$$
\sigma h_{C}^{1 / 2}\left\|\bar{R}_{C}\right\|_{L^{2}(C)}=\int_{C} \bar{R}_{C} d s=\int_{C} \bar{R}_{C} v_{C} d s=\int_{C}\left(\bar{R}_{C}-R_{C}\right) v_{C} d s+\int_{C} R_{C} v_{C} d s
$$

The first term of the last line equals $\operatorname{osc}\left(R_{C}, C\right)$. The second term reads

$$
\int_{C} R_{C} v_{C} d s=R\left(v_{C}\right)-\int_{\omega_{C}} R_{\mathscr{T}} v_{C} d x=R\left(v_{C}\right)-\int_{\omega_{C}}\left(R_{\mathscr{T}}-\bar{R}_{\mathscr{T}}\right) v_{C} d x
$$

With $\gamma_{C}:=h_{C}^{1 / 2}\left\|R_{C}\right\|_{L^{2}(C)}$ the estimator $\eta_{\ell}$ is bounded by

$$
\begin{aligned}
\eta_{\ell}^{2} & =\sum_{C \in \mathscr{C}_{\ell}} h_{C}^{1 / 2} \gamma_{C}\left\|R_{C}\right\|_{L^{2}(C)} \\
& \leq \sum_{C \in \mathscr{C}_{\ell}} R\left(\gamma_{C} v_{C}\right)+2 \sum_{C \in \mathscr{C}_{\ell}} \gamma_{C} \operatorname{osc}\left(R_{C}, C\right)+\sum_{C \in \mathscr{C}_{\ell}} \gamma_{C} \operatorname{osc}\left(R_{\mathscr{T}}, \mathscr{T}_{\ell}\left(\omega_{C}\right)\right) \\
& \lesssim\|R\|_{V^{*}}\left\|\sum_{C \in \mathscr{C}_{\ell}} \gamma_{C} v_{C}\right\|_{V}+\left(\sum_{C \in \mathscr{C}_{\ell}} \gamma_{C}^{2}\right)^{1 / 2}\left(\operatorname{osc}\left(R_{\mathscr{C}}, \mathscr{C}_{\ell}\right)+\operatorname{osc}\left(R_{\mathscr{T}}, \mathscr{T}_{\ell}\right)\right) .
\end{aligned}
$$

Noting that $\left\|\sum_{C \in \mathscr{C}_{\ell}} \gamma_{C} v_{C}\right\|_{V} \lesssim \eta_{\ell}=\left(\sum_{C \in \mathscr{C}_{\ell}} \gamma_{C}^{2}\right)^{1 / 2}$ yields the desired result

$$
\eta_{\ell} \lesssim\|R\|_{V^{*}}+\operatorname{osc}\left(R_{\mathscr{C}}, \mathscr{C}_{\ell}\right)+\operatorname{osc}\left(R_{\mathscr{T}}, \mathscr{T}\right)
$$

\section{Poisson Problem}

This section is devoted to conforming, non-conforming and mixed finite element discretisations for the Poisson model problem of Section 2.2.

\subsection{Conforming Finite Element Methods}

Conforming methods with a discrete space $V_{\ell} \subset V=H_{0}^{1}(\Omega)$ approximate the flux $p=\nabla u$ by $p_{\ell}:=\nabla u_{\ell}$. The discrete problem reads: Find $u_{\ell} \in V_{\ell}$ such that for all $v_{\ell} \in V_{\ell}$

$$
\mathscr{A}\left(\nabla u_{\ell}, u_{\ell}\right)\left(\nabla v_{\ell}, v_{\ell}\right)=\int_{\Omega} \nabla u_{\ell} \cdot \nabla v_{\ell} d x=\int_{\Omega} f v_{\ell} d x=\ell\left(\nabla v_{\ell}, v_{\ell}\right) .
$$

Note that the consistency residual vanishes, i.e., $\mathscr{R} e s_{\text {Cons }}=\mathscr{R} e s_{Q}=0$.

In order to estimate the equilibrium residual (3.4), we use Theorem 5.2. Let $V_{\ell}=P_{k}\left(\mathscr{T}_{\ell}\right) \cap$ $V$. An elementwise integration by parts and the product rule

$$
\left[v p_{\ell} \cdot v_{E}\right]_{E}=\left[p_{\ell} \cdot v_{E}\right]_{E}\langle v\rangle_{E}+\left\langle p_{\ell} \cdot v_{e}\right\rangle_{E}[v]_{E}=\left[p_{\ell} \cdot v_{E}\right]_{E}\langle v\rangle_{E}
$$


imply

$$
\begin{aligned}
\mathscr{R}_{e s_{\mathrm{eq}}}(v)=\mathscr{R} e s_{V}(v) & =\int_{\Omega} f v d x-\int_{\Omega} p_{\ell} \cdot \nabla v d x \\
& =\sum_{T \in \mathscr{T}_{\ell}} \int_{T}\left(f+\operatorname{div} p_{\ell}\right) v d x+\sum_{E \in \mathscr{E}_{\ell}} \int_{E}\left[p_{\ell} \cdot v_{E}\right]_{E}\langle v\rangle_{E} d s .
\end{aligned}
$$

Hence, the residual allows the form (5.3) with the local volume and edge terms of the explicit residual on triangles $T \in \mathscr{T}_{\ell}$ and edges $E \in \mathscr{E}_{\ell}$ by

$$
R_{T}:=\left.f\right|_{T}+\left.\operatorname{div} p_{\ell}\right|_{T} \quad \text { and } \quad R_{E}:=\left[p_{\ell} \cdot v_{E}\right]_{E} .
$$

The global residuals then read

$$
R_{\mathscr{T}}:=f+\operatorname{div}_{\ell} p_{\ell} \quad \text { and } \quad R_{\mathscr{E}}:=\left[p_{\ell} \cdot v_{\mathscr{E}}\right]_{\mathscr{E}}
$$

The equilibrium residual $\mathscr{R} e s_{V}$ has the form of Theorem 5.2 and $V_{\ell} \subset \operatorname{ker} \mathscr{R} e s_{V}$. Moreover, Assumption 5.1 holds with $\Pi=\mathrm{id}_{V_{\ell}}$. This leads to the error estimator

$$
\eta_{\ell}=\left\|h_{\mathscr{E}}^{1 / 2} R_{\mathscr{E}}\right\|_{L^{2}\left(\bigcup \mathscr{E}_{\ell}\right)}
$$

and to the error estimations

$$
\begin{aligned}
\left\|u-u_{\ell}\right\|_{V} \approx\left\|\mathscr{R} e s_{\mathrm{eq}}\right\|_{V^{*}} & \lesssim \eta_{\ell}+\operatorname{Osc}\left(R_{\mathscr{T}}, \mathscr{K}_{\ell}\right), \\
\eta_{\ell} & \lesssim\left\|u-u_{\ell}\right\|_{V}+\operatorname{osc}\left(R_{\mathscr{C}}, \mathscr{C}_{\ell}\right)+\operatorname{osc}\left(R_{\mathscr{T}}, \mathscr{T}_{\ell}\right) .
\end{aligned}
$$

In case of the affine discrete space $V_{\ell}=P_{1}\left(\mathscr{T}_{\ell}\right)$ for $m=1, \operatorname{div} \nabla u_{\ell}=\left.\overline{\operatorname{div} p_{\ell}}\right|_{T}=0$ and the error estimator simplifies to

$$
\left\|u-u_{\ell}\right\| \lesssim \eta_{\ell}+\operatorname{Osc}\left(f, \mathscr{K}_{\ell}\right) \text { and } \eta_{\ell} \lesssim\left\|u-u_{\ell}\right\|_{V}+\operatorname{osc}\left(R_{\mathscr{C}}, \mathscr{C}_{\ell}\right)+\operatorname{osc}\left(f, \mathscr{T}_{\ell}\right)
$$

\subsection{Non-Conforming Finite Element Methods $\left(\mathrm{CR}_{1}\left(\mathscr{T}_{\ell}\right)\right)$}

In the non-conforming Crouzeix-Raviart FEM, the discrete solution $u_{\ell} \in V_{\ell}^{\mathrm{nc}}=\mathrm{CR}_{1}\left(\mathscr{T}_{\ell}\right)$ solves

$$
\mathscr{A}_{\ell}\left(\nabla_{\ell} u_{\ell}, u_{\ell}\right)=\ell_{Q}+\ell_{V}
$$

This is equivalent to the formulation: Seek $u_{\ell} \in \mathrm{CR}_{1}\left(\mathscr{T}_{\ell}\right)$ with

$$
\int_{\Omega} \nabla_{\ell} u_{\ell} \cdot \nabla_{\ell} v_{\ell} d x=\int_{\Omega} f v_{\ell} d x \text { for all } v_{\ell} \in V_{\ell}^{\mathrm{nc}}\left(\mathscr{T}_{\ell}\right) .
$$

Recall that the $\nabla_{\ell}$ denotes the piecewise action of the gradient operator with respect to the triangulation $\mathscr{T}_{\ell}$. 
Theorem 5.1 yields equivalence of the explicit consistency error estimators for $\Omega \subset \mathbb{R}^{2}$,

$$
\begin{aligned}
& \mu_{1}:=\left\|h_{\mathscr{T}}^{-1}\left(u_{\ell}-A_{\mathscr{T}_{\ell}} u_{\ell}\right)\right\|_{L^{2}(\Omega)}=\left(\sum_{T \in \mathscr{T}_{\ell}}\left\|h_{T}^{-1}\left(u_{\ell}-A_{\mathscr{T}_{\ell}} u_{\ell}\right)\right\|_{L^{2}(T)}^{2}\right)^{1 / 2}, \\
& \mu_{2}:=\left\|h_{\mathscr{E}}^{1 / 2}\left[\nabla u_{\ell} \cdot \tau_{\mathscr{E}}\right]_{\mathscr{E}_{\ell}}\right\|_{L^{2}\left(\bigcup \mathscr{E}_{\ell}\right)}=\left(\sum_{E \in \mathscr{E}_{\ell}} h_{E}\left\|\left[\nabla u_{\ell} \cdot \tau_{E}\right]_{E}\right\|_{L^{2}(E)}^{2}\right)^{1 / 2}, \\
& \mu_{3}:=\left\|h_{\mathscr{E}}^{-1 / 2}\left[u_{\ell}\right]_{\mathscr{E}_{\ell}}\right\|_{L^{2}\left(\bigcup \mathscr{E}_{\ell}\right)}=\left(\sum_{E \in \mathscr{E}_{\ell}} h_{E}^{-1}\left\|\left[u_{\ell}\right]_{E}\right\|_{L^{2}(E)}^{2}\right)^{1 / 2} \cdot
\end{aligned}
$$

The equilibrium estimators from the previous section in the conforming case can be used for $V_{\ell}=\mathrm{CR}_{1}\left(\mathscr{T}_{\ell}\right)$ since all conditions of Theorem 5.2 are satisfied: The residual $\mathscr{R} e s_{V}$ can be written in the form (5.3) with $R_{\mathscr{T}}$ and $R_{\mathscr{E}}$ from (6.1) and it holds $\mathrm{CR}_{1}\left(\mathscr{T}_{\ell}\right) \subset \operatorname{ker} \mathscr{R} e s_{V}$.

Remark 6.1. The references [CHO7, COH07] list examples for non-conforming FEMs where $\Pi$ is not the identity, e.g. the Rannacher-Turek nonconforming FEM for rotated polynomials on parallelograms where $V_{\ell}^{c} \not \subset V_{\ell}^{n c}$.

\subsection{Mixed Finite Element Methods $\left(\mathrm{RT}_{0}\right)$}

In the mixed Raviart-Thomas FEM of the Poisson model problem (2.3), one seeks the discrete solution $\left(p_{\ell}, u_{\ell}\right) \in Q_{\ell} \times V_{\ell}:=\mathrm{RT}_{0}\left(\mathscr{T}_{\ell}\right) \times P_{0}\left(\mathscr{T}_{\ell}\right)$ for the solution $p:=\nabla u$, i.e.

$$
\begin{aligned}
& \forall q_{\ell} \in Q_{\ell} \quad \int_{\Omega} p_{\ell} \cdot q_{\ell} d x-\int_{\Omega} q_{\ell} \cdot \nabla u_{\ell} d x=0 \\
& \forall v_{\ell} \in V_{\ell} \quad \int_{\Omega} p_{\ell} \cdot \nabla v_{\ell} d x=\int_{\Omega} f v_{\ell} d x .
\end{aligned}
$$

By the Helmholtz decomposition in $n=2$ or $n=3$ dimensions, there exist functions $\alpha \in V=H_{0}^{1}(\Omega)$ and $\beta \in H^{1}(\Omega)$ with

$$
p_{\ell}=\nabla \alpha+\operatorname{Curl} \beta \quad \text { and } \quad \operatorname{dist}_{L^{2}(\Omega)}\left(p_{\ell}, \nabla H_{0}^{1}(\Omega)\right)=\|\operatorname{Curl} \beta\|_{L^{2}(\Omega)} .
$$

We utilize the different definitions of the curl differential operator given in Section 1. With $\tilde{u}_{\ell}=\alpha$ it holds, for any $q_{\ell} \in Q_{\ell}$,

$$
\mathscr{R} e s_{Q}\left(q_{\ell}\right)=-\int_{\Omega}\left(p_{\ell}-\nabla \alpha\right) \cdot q_{\ell} d x=\int_{\Omega} \operatorname{Curl} \beta \cdot q_{\ell} d x .
$$

The residual (6.4) does not satisfy the conditions in Theorem 5.1 since $p_{\ell} \in \mathrm{RT}_{0}\left(\mathscr{T}_{\ell}\right)$ does not belong to the space of piecewise gradients $P_{0}\left(\mathscr{T}_{\ell}\right)$. It holds

$$
\left\|p-p_{\ell}\right\|_{L^{2}(\Omega)}^{2} \approx\left\|\mathscr{R} e s_{Q}\right\|_{Q_{\ell}^{*}}^{2}=\|\operatorname{Curl} \beta\|_{L^{2}(\Omega)}^{2}
$$


and the orthogonality $\operatorname{Curl} \beta \perp_{L^{2}(\Omega)} \nabla \alpha$ implies

$$
\|\operatorname{Curl} \beta\|_{L^{2}(\Omega)}^{2}=\int_{\Omega} \operatorname{Curl} \beta \cdot(\operatorname{Curl} \beta+\nabla \alpha) d x=\int_{\Omega} \operatorname{Curl} \beta \cdot p_{\ell} d x .
$$

For any function $\beta_{\ell} \in P_{1,0}\left(\mathscr{T}_{\ell}\right)$ with $\operatorname{Curl} \beta_{\ell} \in P_{0}\left(\mathscr{T}_{\ell} ; \mathbb{R}^{n}\right)$ it holds

$$
\operatorname{div} \operatorname{Curl} \beta_{\ell}=0 \quad \text { a.e. in } \Omega
$$

and it is an admissible test function in the RT-FEM. To obtain an explicit consistency error estimator, calculate

$$
\begin{aligned}
\int_{\Omega} \operatorname{Curl} \beta \cdot p_{\ell} & =\int_{\Omega} \operatorname{Curl}\left(\beta-\beta_{\ell}\right) \cdot p_{\ell} d x \\
& =\sum_{T \in \mathscr{T}_{\ell}}-\int_{T} \operatorname{curl} p_{\ell}\left(\beta-\beta_{\ell}\right) d x+\int_{\partial T} p_{\ell}\left(\beta-\beta_{\ell}\right) \cdot \tau_{\partial T} d s \\
& =-\int_{\Omega} \operatorname{curl}_{\ell} p_{\ell}\left(\beta-\beta_{\ell}\right) d x+\sum_{E \in \mathscr{E}_{\ell}} \int_{E}\left(\beta-\beta_{\ell}\right)\left[p_{\ell} \cdot \tau_{E}\right]_{E} d s
\end{aligned}
$$

and choose $\beta_{\ell}=J_{\ell} \beta$ for the approximation operator $J_{\ell}$ (cf. Definition 4.1 and [Car99]) to obtain

$$
\left\|\mathscr{R} e s_{\text {cons }}\right\|_{Q^{*}}^{2} \lesssim\|\nabla \beta\|_{L^{2}(\Omega)}\left(\left\|h_{\mathscr{T}} \operatorname{curl}_{\ell} p_{\ell}\right\|_{L^{2}(\Omega)}+\left\|h_{\mathscr{E}}^{1 / 2}\left[p_{\ell} \cdot \tau_{\mathscr{E}}\right]_{\mathscr{E}_{\ell}}\right\|_{L^{2}\left(\bigcup \mathscr{E}_{\ell}\right)}\right) .
$$

With $\|\beta\|_{H^{1}(\Omega)} \lesssim\left\|p-p_{\ell}\right\|_{L^{2}(\Omega)}$, it follows that

$$
\left\|p-p_{\ell}\right\|_{L^{2}(\Omega)} \lesssim \mu_{\ell}:=\left\|h_{\mathscr{T}} \operatorname{curl}_{\ell} p_{\ell}\right\|_{L^{2}(\Omega)}+\left\|h_{\mathscr{E}}^{1 / 2}\left[p_{\ell} \cdot \tau_{\mathscr{E}}\right]_{\mathscr{E}_{\ell}}\right\|_{L^{2}\left(\bigcup \mathscr{E}_{\ell}\right)}
$$

For the analysis of the efficiency of the error estimator we refer to [Car97].

For the evaluation of $\mathscr{R} e s_{V}$, note that $p_{\ell} \in H(\operatorname{div}, \Omega)$ and define $\bar{f}_{\ell} \in P_{0}\left(\mathscr{T}_{\ell}\right)$ satisfying $\left.\bar{f}_{\ell}\right|_{T}=|T|^{-1} \int_{T} f d x$ on every triangle $T \in \mathscr{T}_{\ell}$. Then, for all $v \in H_{0}^{1}(\Omega)$ and $\bar{v}_{\ell} \in P_{0}\left(\mathscr{T}_{\ell}\right)$ analogous to $\bar{f}_{\ell}$, it follows

$$
\begin{aligned}
\mathscr{R e s}_{\mathrm{eq}}(v)=\mathscr{R} e s_{V}(v) & :=\int_{\Omega} f v d x-\int_{\Omega} p_{\ell} \cdot \nabla v d x \\
& =\int_{\Omega}\left(f+\operatorname{div} p_{\ell}\right) v d x=\int_{\Omega}\left(f-\bar{f}_{\ell}\right)\left(v-\bar{v}_{\ell}\right) d x .
\end{aligned}
$$

For the norms it then holds

$$
\left\|\mathscr{R} e s_{\mathrm{eq}}(v)\right\|_{L^{2}(\Omega)} \leq \sum_{T \in \mathscr{T}}\left\|f-\bar{f}_{\ell}\right\|_{L^{2}(T)}\left\|v-\bar{v}_{\ell}\right\|_{L^{2}(T)} .
$$


On every $T \in \mathscr{T}$, a Poincaré inequality with the Payne-Weinberger constant $1 / \pi$ [PW60] for $v-\bar{v} \in H_{0}^{1}(T)$ yields

$$
\left\|v-\bar{v}_{\ell}\right\|_{L^{2}(T)} \leq \frac{1}{\pi}\|\nabla v\|_{L^{2}(T)} \leq\|v\|_{H^{1}(T)}
$$

These considerations lead to the result

$$
\left\|\mathscr{R} e s_{\mathrm{eq}}\right\|_{V^{*}} \lesssim \operatorname{osc}\left(f, \mathscr{T}_{\ell}\right)
$$

Alternatively, from (6.5) it follows with $R_{\mathscr{T}}:=f+\operatorname{div} p_{\ell}$ and $R_{\mathscr{E}}=\left[p_{\ell} \cdot v_{E}\right]_{\mathscr{E}}=0$ for $p_{\ell} \in \operatorname{RT}_{0}\left(\mathscr{T}_{\ell}\right)$ that Theorem 5.2 yields an equilibrium estimator $\eta_{\ell}=\left\|h_{\mathscr{E}}^{1 / 2} R_{\mathscr{E}}\right\|_{L^{2}\left(\bigcup \mathscr{E}_{\ell}\right)}=0$ with

$$
\left\|\mathscr{R} e s_{\mathrm{eq}}\right\|_{V^{*}} \lesssim \operatorname{Osc}\left(R_{\mathscr{T}}, \mathscr{K}_{\ell}\right)
$$

\section{Stokes Problem}

\subsection{Mixed Conforming Finite Element Methods}

The conforming discrete problem of the Stokes equations in the symmetric formulation reads: Find $u_{\ell} \in V_{\ell}$ and $p_{\ell} \in Q_{\ell}$ such that

$$
\begin{array}{ll}
\forall v_{\ell} \in V_{\ell} & \int_{\Omega} 2 \mu \varepsilon\left(u_{\ell}\right): \varepsilon\left(v_{\ell}\right) d x-\int_{\Omega} p_{\ell} \operatorname{div} v_{\ell} d x=\int_{\Omega} f \cdot v_{\ell} d x, \\
\forall q_{\ell} \in Q_{\ell} & \int_{\Omega} q_{\ell} \operatorname{div} u_{\ell} d x=0 .
\end{array}
$$

Recall from Section 2.3 that

$$
\left\|\mathscr{R} e s_{\text {cons }}\right\|_{Q_{\ell}^{*}} \approx 2 \mu\left\|\varepsilon_{\ell}\left(u_{\ell}\right)-\varepsilon_{\ell}\left(\tilde{u}_{\ell}\right)\right\|_{L^{2}\left(\Omega ; \mathbb{R}^{n \times n}\right)}+\left\|\operatorname{div} \tilde{u}_{\ell}\right\|_{L_{0}^{2}(\Omega)}
$$

In the conforming case, $\tilde{u}_{\ell}=u_{\ell}$ implies $\left\|\varepsilon\left(u_{\ell}\right)-\varepsilon\left(\tilde{u}_{\ell}\right)\right\|_{L^{2}(\Omega)}=0$ and $\tilde{u}_{\ell}$ is divergence free by construction (the same holds in the non-symmetrical formulation). Hence, the consistency residual vanishes, $\left\|\mathscr{R} e s_{\text {cons }}\right\|_{Q_{\ell}^{*}}=0$.

The equilibrium residual reads

$$
\mathscr{R} e s_{\mathrm{eq}}(v):=\int_{\Omega} f \cdot v d x+\int_{\Omega} \sigma_{\ell}: \varepsilon(v) d x
$$


A piecewise integration by parts argument yields

$$
\begin{aligned}
{\mathscr{R} e s_{\mathrm{Eq}}(v)}=\int_{\Omega} f \cdot v d x-\int_{\Omega} \varepsilon(v): \sigma_{\ell} d x \\
=\int_{\Omega} f \cdot v d x-\int_{\Omega} \sigma_{\ell}: \nabla v d x \\
=\int_{\Omega} f \cdot v d x+\sum_{T \in \mathscr{T}_{\ell}}\left(\int_{T} \operatorname{div}_{\ell} \sigma_{\ell} \cdot v d x-\int_{\partial T} \sigma_{\ell} \cdot v_{T} v d s\right) \\
=\sum_{T \in \mathscr{T}_{\ell}} \int_{T}\left(f+\operatorname{div}_{\ell} \sigma_{\ell}\right) \cdot v d x+\sum_{E \in \mathscr{E}_{\ell}} \int_{E}\left[\sigma_{\ell} \cdot v_{E}\right]_{E} \cdot v d s .
\end{aligned}
$$

Hence, in order to apply Theorem 5.2, we set

$$
R_{\mathscr{T}}:=f+\operatorname{div}_{\ell} \sigma_{\ell} \quad \text { and } \quad R_{\mathscr{E}}:=\left[\sigma_{\ell} \cdot v_{\mathscr{E}}\right]_{\mathscr{E}_{\ell}}
$$

With the identity $\Pi$, one derives the reliable equilibrium estimator

$$
\eta_{\ell}^{\mathrm{eq}}:=\operatorname{Osc}\left(R_{\mathscr{T}}, \mathscr{K}_{\ell}\right)+\left\|h_{\mathscr{E}}^{1 / 2}\left[\sigma_{\ell} \cdot v_{\mathscr{E}}\right]_{\mathscr{E}_{\ell}}\right\|_{L^{2}\left(\bigcup \mathscr{E}_{\ell}\right)}
$$

for the symmetrical as well as for the non-symmetrical formulation of the Stokes problem.

Remark 7.1 (Taylor-Hood Elements in 2D). The Taylor-Hood FEM for the non-symmetrical Stokes equations uses $P_{2}$-elements for both components of $u_{\ell}$ and $P_{1}$-elements for the ap-

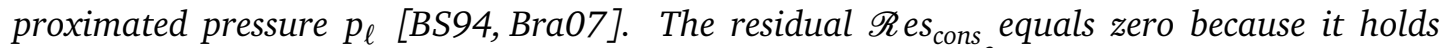
$\operatorname{div} u_{\ell} \in P_{1}\left(\mathscr{T}_{\ell}\right)$ and for all (piecewise affine) $q_{\ell} \in Q_{\ell}$ it holds $\int_{\Omega} q_{\ell} \operatorname{div} u_{\ell} d x=0$. Therefore $\left\|\mathscr{R} e s_{Q}\right\|_{Q^{*}}=\left\|\operatorname{div} u_{\ell}\right\|_{L^{2}(\Omega)}=0$ and the error estimator

$$
\eta_{\ell}:=\eta_{e q}=\operatorname{Osc}\left(R_{\mathscr{T}}, \mathscr{K}_{\ell}\right)+\left\|h_{\mathscr{E}}^{1 / 2}\left[\sigma_{\ell} \cdot v_{\mathscr{E}}\right]_{\mathscr{E}_{\ell}}\right\|_{L^{2}\left(\bigcup \mathscr{E}_{\ell}\right)}
$$

is reliable and efficient for the overall error $\left\|\mathscr{R} e s_{\text {eq }}\right\|+\left\|\mathscr{R} e s_{\text {cons }}\right\|$.

Remark 7.2 (MINI elements in 2D). For the affine nodal basis functions $\varphi_{z}$ (cf. Section 5) the triangle bubble function $b_{T}$ for $T=\operatorname{conv}\{a, b, c\} \in \mathscr{T}_{\ell}$ is defined by

$$
b_{T}:=\varphi_{a} \varphi_{b} \varphi_{c}=\prod_{z \in \mathscr{N}(T)} \varphi_{z}
$$

The MINI-FEM in two dimensions uses the discrete spaces [BS94, Bra07]

$$
V_{\ell}=\left(P_{1,0}\left(\mathscr{T}_{\ell}\right)+\operatorname{span}\left\{b_{T} \mid T \in \mathscr{T}_{\ell}\right\}\right)^{2} \quad \text { and } \quad Q_{\ell}=P_{1,0}\left(\mathscr{T}_{\ell} ; \mathbb{R}^{2}\right) .
$$

The term $\left\|\operatorname{div} u_{\ell}\right\|_{L^{2}(\Omega)}$ is not necessarily zero and therefore, its norm has to be added to the error estimator. The value of

$$
\eta_{\ell}:=\eta_{e q}+\left\|\operatorname{div} u_{\ell}\right\|_{L^{2}(\Omega)}=\operatorname{Osc}\left(R_{\mathscr{T}}, \mathscr{K}_{\ell}\right)+\left\|h_{\mathscr{E}}^{1 / 2}\left[\sigma_{\ell} \cdot v_{\mathscr{E}}\right]_{\mathscr{E}_{\ell}}\right\|_{L^{2}\left(\bigcup \mathscr{E}_{\ell}\right)}+\left\|\operatorname{div} u_{\ell}\right\|_{L^{2}(\Omega)}
$$

is a reliable and efficient estimator for the error norm. 


\subsection{Non-Conforming Finite Element Methods}

The equilibrium error estimator of Section 7.1 can also be employed in the non-conforming case.

For the norm of the consistency residual in the non-symmetric formulation of the Stokes equations as well as (up to a constant) in the symmetric case (7.1), we get

$$
\left\|\mathscr{R} e s_{\text {cons }}\right\|_{V^{*}} \approx\left\|\nabla_{\ell}\left(u_{\ell}\right)-\nabla\left(\tilde{u}_{\ell}\right)\right\|_{Q}+\left\|\operatorname{div} \tilde{u}_{\ell}\right\|_{Q} .
$$

The second term on the right-hand side equals zero since $\tilde{u}_{\ell}$ is chosen to be divergence-free. Then, component-wise application of Theorem 5.1 provides error estimates for example for the finite-element solution in $\left(\mathrm{CR}_{1} \times \mathrm{CR}_{1}\right) \times P_{0}$ for the symmetric Stokes equations,

$$
\mu_{1}:=\left\|h_{\mathscr{E}}^{1 / 2}\left[\nabla u_{\ell} \cdot \tau_{\mathscr{E}}\right]_{\mathcal{E}_{\ell}}\right\|_{L^{2}\left(\bigcup \mathscr{E}_{\ell} ; \mathbb{R}^{2}\right)}, \quad \quad \mu_{2}:=\left\|h_{\mathscr{E}}^{-1 / 2}\left[u_{\ell}\right]_{\mathscr{E}_{\ell}}\right\|_{L^{2}\left(\bigcup \mathscr{E}_{\ell} ; \mathbb{R}^{2}\right)} .
$$

Another example is the finite element $\left(P_{1,0}\left(\mathscr{T}_{\ell} ; \mathbb{R}\right) \times \mathrm{CR}_{1}\right) \times P_{0}$ of Kouhia and Stenberg [KS95] for non-symmetric Stokes which is only non-conforming in one component. Hence, Theorem 5.1 again yields error estimates, with $u_{\ell}=\left(u_{\ell, 1}, u_{\ell, 2}\right)$ for example

$$
\mu_{1}:=\left\|h_{\mathscr{E}}^{1 / 2}\left[\nabla u_{\ell, 2} \cdot \tau_{\mathscr{E}}\right]_{\mathscr{E}_{\ell}}\right\|_{L^{2}\left(\bigcup \mathscr{E}_{\ell}\right)}, \quad \quad \mu_{2}:=\left\|h_{\mathscr{E}}^{-1 / 2}\left[u_{\ell, 2}\right]_{\mathscr{E}_{\ell}}\right\|_{L^{2}\left(\bigcup \mathscr{E}_{\ell}\right)} .
$$

\section{Lamé Problem}

\subsection{Conforming Finite Element Methods}

Conforming methods with a discrete finite element space $V_{\ell} \subset V$ approximate the stress matrix $\sigma=\mathbb{C} \varepsilon(u)$ by $\sigma_{\ell}:=\mathbb{C} \varepsilon\left(u_{\ell}\right)$ and the discrete problem therefore turns out to solve the weak formulation of the Lamé problem: Find the Galerkin solution $u_{\ell} \in V_{\ell}$ such that

$$
\mathscr{A}\left(\mathbb{C} \varepsilon\left(u_{\ell}\right), u_{\ell}\right)=\ell_{Q}+\ell_{V} .
$$

This is equivalent to the mixed formulation of the problem: Find $u_{\ell} \in V_{\ell}$ and $p_{\ell} \in Q_{\ell}$ such that

$$
\begin{array}{cc}
\forall \tau_{\ell} \in Q_{\ell} & \int_{\Omega}\left(\mathbb{C}^{-1} \sigma_{\ell}-\varepsilon\left(u_{\ell}\right)\right): \tau_{\ell} d x=0, \\
\forall v_{\ell} \in V_{\ell} & \int_{\Omega} \varepsilon\left(v_{\ell}\right): \sigma_{\ell} d x=-\int_{\Omega} \operatorname{div} \sigma_{\ell} \cdot v_{\ell} d x=\int_{\Omega} f \cdot v_{\ell} d x .
\end{array}
$$

In the conforming case considered first, the previous formulation is identical to

$$
-\int_{\Omega} \operatorname{div} \mathbb{C} \varepsilon\left(u_{\ell}\right) \cdot v_{\ell} d x=\int_{\Omega} f \cdot v_{\ell} d x \quad \text { for all } v_{\ell} \in V_{\ell} .
$$

The consistency residual vanishes for any conforming finite element method, i.e. $\mathscr{R} e s_{\text {cons }}=$ $\mathscr{R} e s_{Q}=0$. 
Let $V_{\ell}=P_{k}\left(\mathscr{T}_{\ell} ; \mathbb{R}^{n}\right)$. In analogy to the derivation of the explicit estimator in Section 7.1, noting that $\sigma_{\ell}:=\mathbb{C} \varepsilon\left(u_{\ell}\right)$ is symmetric and using integration by parts, the equilibrium residual $\mathscr{R} e s_{\text {eq }}=\mathscr{R} e s_{V}$ is defined by (3.6) and can be written as in (5.3) with

$$
R_{\mathscr{T}}:=f+\operatorname{div}_{\ell} \sigma_{\ell} \quad \text { and } \quad R_{\mathscr{E}}:=\left[\sigma_{\ell} \cdot v_{\mathscr{E}}\right]_{\mathscr{E}_{\ell}}
$$

This is identical to (7.2) and again can be used with Theorem 5.2 and $\Pi=\mathrm{id}_{V_{\ell}}$ which yields the estimator (7.3). Moreover, from $\|e\|_{H^{1}(\Omega)} \lesssim\left\|\sigma-\sigma_{\ell}\right\|_{L^{2}(\Omega)}$ one obtains

$$
\left\|\sigma-\sigma_{\ell}\right\|_{L^{2}(\Omega)} \approx \eta_{\ell}
$$

independent of the material parameter $\lambda$.

Remark 8.1 $\left(P_{k} \times P_{k}\right)$. For any conforming finite element method of arbitrary polynomial degree the usual equilibrium estimator given above is applicable.

\subsection{Non-Conforming Finite Element Methods}

There are different robust non-conforming finite element methods known for elasticity problems. As an example we consider a construction due to Kouhia and Stenberg [KS95] which leads to the discrete space $V_{\ell}:=P_{1,0}\left(\mathscr{T}_{\ell}\right) \times \mathrm{CR}_{1,0}\left(\mathscr{T}_{\ell}\right) \subset H_{0}^{1}(\Omega) \times H^{1}\left(\mathscr{T}_{\ell}\right)$. The discrete problem reads: Find $u_{\ell} \in V_{\ell}$ s.t.

$$
\int_{\Omega} v_{\ell} \cdot \operatorname{div} \mathbb{C} \varepsilon_{\ell}\left(u_{\ell}\right) d x=\int_{\Omega} f \cdot v_{\ell} d x \quad \text { for all } v_{\ell} \in V_{\ell} .
$$

The discrete stress is given by $\sigma_{\ell}:=\mathbb{C} \varepsilon_{\ell}\left(u_{\ell}\right)$ and while the equilibrium error is estimated by $\eta_{\ell}$ as in the conforming case above, the consistency residual $\mathscr{R} e s_{\text {cons }}=\mathscr{R} e s_{Q}=\varepsilon_{\ell}\left(u-\tilde{u}_{\ell}\right)$ does not vanish in the second component and also has to be considered. Since $u_{\ell}$ satisfies the requirements of Theorem 5.1, two equivalent reliable consistency estimators are

$$
\begin{aligned}
& \mu_{1}:=\left\|h_{\mathscr{E}}^{1 / 2}\left[\nabla u_{\ell} \cdot \tau_{\mathscr{E}}\right]_{\mathscr{E}_{\ell}}\right\|_{L^{2}\left(\bigcup \mathscr{E}_{\ell}\right)}=\left(\sum_{E \in \mathscr{E}_{\ell}} h_{E}\left\|\left[\nabla u_{\ell} \cdot \tau_{E}\right]_{E}\right\|_{L^{2}(E)}^{2}\right)^{1 / 2}, \\
& \mu_{2}:=\left\|h_{\mathscr{E}}^{-1 / 2}\left[u_{\ell}\right]_{\mathscr{E}_{\ell}}\right\|_{L^{2}\left(\bigcup \mathscr{E}_{\ell}\right)}=\left(\sum_{E \in \mathscr{E}_{\ell}} h_{E}^{-1}\left\|\left[u_{\ell}\right]_{E}\right\|_{L^{2}(E)}^{2}\right)^{1 / 2} .
\end{aligned}
$$

\subsection{Mixed Finite Element Methods}

\subsubsection{Plane Elasticity Element with Reduced Symmetry (PEERS)}

Some $\lambda$-robust approximation of the solution of the mixed formulation concerns discrete spaces of reduced symmetry. For a possibly non-symmetric matrix-valued $L^{2}$-function $A$, we denote the skew-symmetric part of $A$ by

$$
\text { skew } A=A-\operatorname{sym} A=\left(A-A^{T}\right) / 2 \text { and } \mathbb{R}_{\text {skew }}^{d \times d}=\left\{B \in \mathbb{R}^{d \times d} \mid B+B^{T}=0\right\} .
$$


Notice that for a symmetric matrix $A \in \mathbb{R}_{\text {sym }}^{d \times d}$ and for a skew-symmetric matrix $B \in \mathbb{R}_{\text {skew }}^{d \times d}$ it holds $A: B=0$.

The idea behind the PEERS [ABD84] is to allow a non-symmetric stress matrix $\sigma$. In our notation, a tuple $(\sigma, \gamma)$ denotes such a possibly non-symmetric stress matrix $\sigma$ and a skew-symmetric matrix $\gamma$. The equation

$$
\int_{\Omega} \sigma: \gamma d x=0 \text { for all } \gamma \text { in some subset of } L^{2}\left(\Omega ; \mathbb{R}_{\text {skew }}^{d \times d}\right)
$$

guarantees the symmetry in weak form of $\sigma$. The spaces

$$
Q:=L^{2}\left(\Omega ; \mathbb{R}^{2}\right) \times W, W:=L^{2}\left(\Omega ; \mathbb{R}_{\text {skew }}^{2 \times 2}\right) \text { and } V:=H\left(\operatorname{div}, \Omega ; \mathbb{R}^{2 \times 2}\right)
$$

and the bilinear forms

$$
\begin{aligned}
a(u, \gamma ; v, \delta) & :=\int_{\Omega}(u \cdot v+\gamma: \delta) d x \\
b(u, \gamma ; \sigma) & =\int_{\Omega}(u \cdot \operatorname{div} \sigma+\operatorname{skew} \sigma: \gamma) d x, \\
c(\sigma, \tau) & :=\int_{\Omega} \mathbb{C}^{-1} \sigma: \tau d x \\
\Lambda(\sigma) & :=(\operatorname{div} \sigma, \operatorname{skew} \sigma), \\
\ell_{Q}(u, \gamma ; v, \delta) & :=\int_{\Omega}(f \cdot v+u \cdot v+\gamma: \delta) d x, \\
\ell_{V}(\sigma) & :=0
\end{aligned}
$$

lead to a mixed formulation which includes an additional equation for the weak symmetry condition, i.e.

$$
\begin{array}{lrl}
\forall v \in L^{2}\left(\Omega ; \mathbb{R}^{2}\right) & -\int_{\Omega} \operatorname{div} \sigma \cdot v d x=\int_{\Omega} f \cdot v d x, \\
\forall \delta \in W & \int_{\Omega} \operatorname{skew} \sigma: \delta d x=0, \\
\forall \tau \in V & \int_{\Omega}\left(\operatorname{div} \tau \cdot u+\gamma: \operatorname{skew} \tau+\mathbb{C}^{-1} \sigma: \tau\right) d x=0 .
\end{array}
$$

PEERS employ the discrete spaces

$$
\begin{aligned}
Q_{\ell} & :=P_{0}\left(\mathscr{T}_{\ell} ; \mathbb{R}^{2}\right) \times W_{\ell}, \\
W_{\ell} & :=\left\{\gamma_{\ell} \in L^{2}\left(\Omega ; \mathbb{R}_{\text {sym }}^{2 \times 2}\right) \cap C^{0}\left(\Omega ; \mathbb{R}_{\text {sym }}^{2 \times 2}\right)\left|\forall T \in \mathscr{T}_{\ell}, \quad \gamma_{\ell}\right|_{\mathscr{T}} \in P_{1}\left(T ; \mathbb{R}_{\text {sym }}^{2 \times 2}\right)\right\}, \\
V_{\ell} & :=\left\{\sigma_{\ell} \in H\left(\operatorname{div}, \Omega ; \mathbb{R}^{2 \times 2}\right)\left|\forall T \in \mathscr{T}_{\ell}, \quad \sigma_{\ell}\right|_{T} \in \operatorname{RT}_{0}(T) \oplus B_{0}(T)\right\} .
\end{aligned}
$$


Here, $\mathrm{RT}_{0}$ is the lowest order Raviart-Thomas space and $B_{0}$ is the curl of the bubblefunction space for $b_{T}=\prod_{z \in \mathcal{N}(T)} \varphi_{z}$ defined on any $T \in \mathscr{T}_{\ell}$ by

$$
\begin{aligned}
\mathrm{RT}_{0}(T) & :=\left\{\sigma \in L^{2}\left(T ; \mathbb{R}^{2 \times 2}\right) \mid \sigma(x)=\tau+a \cdot x^{T}, \tau \in \mathbb{R}^{2 \times 2}, a \in \mathbb{R}^{2}\right\} \\
B_{0}(T) & :=\left\{\sigma \in L^{2}\left(T ; \mathbb{R}^{2 \times 2}\right) \mid \sigma(x)=a \cdot \operatorname{Curl} b_{T}(x)^{T}, a \in \mathbb{R}^{2}\right\} .
\end{aligned}
$$

Note that, in general, skew $\sigma_{\ell} \not \equiv 0$ for the PEERS stress field $\sigma_{\ell}$. The following equivalence is due to the residuals from Section 3.5 for any $\tilde{u}_{\ell} \in L^{2}\left(\Omega ; \mathbb{R}^{2}\right)$,

$$
\left\|\sigma-\operatorname{sym} \sigma_{\ell}\right\|_{Q}+\left\|u-\tilde{u}_{\ell}\right\|_{V} \approx\left\|\varepsilon\left(\tilde{u}_{\ell}\right)-\mathbb{C}^{-1} \operatorname{sym} \sigma_{\ell}\right\|_{Q^{*}}+\left\|\mathscr{R} e s_{V}\right\|_{V^{*}}
$$

We remark that in essence the derivation of bounds for the residuals is analogous to the treatment in Section 6.3. To estimate the consistency term

$$
\mu:=\min _{\tilde{u}_{\ell} \in V}\left\|\varepsilon\left(\tilde{u}_{\ell}\right)-\mathbb{C}^{-1} \operatorname{sym} \sigma_{\ell}\right\|_{Q}
$$

a Helmholtz decomposition for some symmetric stress in $L^{2}\left(\Omega ; \mathbb{R}_{\text {sym }}^{2 \times 2}\right)$ has been derived in [CD98] and results in

$$
\mathbb{C}^{-1} \operatorname{sym} \sigma_{\ell}=\varepsilon(\alpha)+\operatorname{Curl} \operatorname{Curl} \beta \quad \text { for some } \alpha \in H_{0}^{1}(\Omega)^{2} \text { and } \beta \in H^{2}(\Omega),
$$

cf. [CD98,CDFH00] for details in slightly different notation. Therefore, the choice of $\tilde{u}_{\ell}=\alpha$ leads to the minimum in (8.4) and leads to orthogonality of $B:=\operatorname{Curl} \beta \in \operatorname{Curl}\left(H^{1}(\Omega)^{2}\right)$ and $D\left(H_{0}^{1}(\Omega)^{2}\right)$

$$
\mu=\min _{\tilde{u}_{\ell} \in V}\left\|\varepsilon\left(\tilde{u}_{\ell}\right)-\mathbb{C}^{-1} \operatorname{sym} \sigma_{\ell}\right\|_{Q}=\|\operatorname{Curl} \operatorname{Curl} \beta\|_{Q}
$$

Let $J_{\ell}: H^{1}(\Omega) \rightarrow P_{1}(\Omega)$ be some quasi-interpolation operator from Section 4.3 , set $B:=$ $\operatorname{Curl} \beta$ and notice that $B_{\ell}:=J_{\ell} B \in \mathrm{RT}_{0}$ is an admissible test tensor in (8.2c) and hence

$$
\int_{\Omega} \operatorname{Curl} B_{\ell}:\left(\mathbb{C}^{-1} \operatorname{sym} \sigma_{\ell}+\gamma_{\ell}\right) d x=0
$$

This, the aforementioned orthogonality and an element-wise integration by parts lead to

$$
\begin{aligned}
\mu^{2}=\|\operatorname{Curl} B\|_{Q}^{2}= & \int_{\Omega} \operatorname{Curl}\left(B-B_{\ell}\right):\left(\mathbb{C}^{-1} \operatorname{sym} \sigma_{\ell}+\gamma_{\ell}\right) d x \\
\leq & \sum_{T \in \mathscr{T}_{\ell}}\left\|B-B_{\ell}\right\|_{L^{2}(T)}\left\|\operatorname{curl}\left(\mathbb{C}^{-1} \operatorname{sym} \sigma_{\ell}+\gamma_{\ell}\right)\right\|_{L^{2}(T)} \\
& +\sum_{E \in \mathscr{E}_{\ell}}\left\|B-B_{\ell}\right\|_{L^{2}(E)}\left\|\left[\mathbb{C}^{-1} \operatorname{sym} \sigma_{\ell}+\gamma_{\ell}\right] \cdot \tau_{E}\right\|_{L^{2}(E)} .
\end{aligned}
$$


Since $\|B\|_{H^{1}(\Omega)} \lesssim\|\operatorname{Curl} B\|_{Q}$ and with the interpolation estimates for $\left\|B-B_{\ell}\right\|$ on triangles and edges, it follows eventually that

$$
\begin{aligned}
\mu^{2} \lesssim & \left(\sum_{E \in \mathscr{E}_{\ell}} h_{E}\left\|\left[\mathbb{C}^{-1} \operatorname{sym} \sigma_{\ell}+\gamma_{\ell}\right] \cdot \tau_{E}\right\|_{L^{2}(E)}^{2}\right)^{1 / 2}+ \\
& \left(\sum_{T \in \mathscr{T}_{\ell}} h_{T}^{2}\left\|\operatorname{curl}\left(\mathbb{C}^{-1} \operatorname{sym} \sigma_{\ell}+\gamma_{\ell}\right)\right\|_{L^{2}(T)}^{2}\right)^{1 / 2} .
\end{aligned}
$$

Recall from (8.2a) that, for $\sigma_{\ell} \in V_{\ell}$, it holds

$$
\int_{\Omega} v_{\ell} \cdot\left(f+\operatorname{div} \sigma_{\ell}\right) d x=0 \quad \text { for all } v_{\ell} \in P_{0}\left(\mathscr{T}_{\ell} ; \mathbb{R}^{2}\right) .
$$

The equilibrium residual $\mathscr{R} e s_{V}$ is split in the symmetric and the skew-symmetric part of $\sigma_{\ell}$ and the observation that $\varepsilon(v): \sigma_{\ell}=\nabla v: \operatorname{sym} \sigma_{\ell}$ plus an integration by parts lead, for all $v \in Q$, to

$$
\begin{aligned}
\mathscr{R}_{e s_{V}}(v) & =\int_{\Omega}\left(f \cdot v-\varepsilon(v): \sigma_{\ell}\right) d x \\
& =\int_{\Omega}\left(f+\operatorname{div} \sigma_{\ell}\right) \cdot v d x-\int_{\Omega} \operatorname{skew}\left(\sigma_{\ell}\right): \nabla v d x .
\end{aligned}
$$

Note that $\sigma_{\ell} \in H(\operatorname{div}, \Omega)$ and thus there are no jumps across inter-element edges. Let $\bar{v}_{\ell}$ and $\left.\bar{f}_{\ell}\right|_{T}=|T|^{-1} \int_{T} \operatorname{div} \sigma_{\ell} d x$ denote the $\mathscr{T}$-piecewise constant averages of $v$ and $f$. Then, following the same arguments as for (6.5), Poincaré inequalities result in

$$
\begin{aligned}
\int_{\Omega}\left(f+\operatorname{div} \sigma_{\ell}\right) \cdot v d x & =-\int_{\Omega}\left(v-\bar{v}_{\ell}\right)\left(f-\bar{f}_{\ell}\right) d x \\
& \leq\left\|h_{\mathscr{T}}^{-1}\left(v-\bar{v}_{\ell}\right)\right\|_{L^{2}(\Omega)}\left\|h_{\mathscr{T}}\left(f-\bar{f}_{\ell}\right)\right\|_{L^{2}(\Omega)} \\
& \leq\|\nabla v\|_{L^{2}(\Omega)} \operatorname{osc}\left(f, \mathscr{T}_{\ell}\right) / \pi .
\end{aligned}
$$

With higher-order oscillations $\operatorname{osc}\left(f, \mathscr{T}_{\ell}\right)$ for $f \in H^{1}\left(\mathscr{T}_{\ell}\right)^{2}$, it follows

$$
\left\|\mathscr{R} e s_{V}\right\|_{V^{*}} \leq\left\|\operatorname{skew} \sigma_{\ell}\right\|_{L^{2}(\Omega)}+\operatorname{osc}\left(f, \mathscr{T}_{\ell}\right) / \pi \text {. }
$$

The non-symmetric stress error of $\sigma_{\ell}$ is split into

$$
\left\|\sigma-\sigma_{\ell}\right\|_{Q}^{2}=\left\|\sigma-\operatorname{sym} \sigma_{\ell}\right\|_{Q}^{2}+\left\|\operatorname{skew} \sigma_{\ell}\right\|_{Q}^{2} .
$$

This amounts to the final a posteriori error estimate

$$
\left\|\sigma-\sigma_{\ell}\right\|_{Q} \lesssim \mu+\left\|\mathscr{R} e s_{Q}\right\|_{V^{*}}+\| \text { skew } \sigma_{\ell} \|_{Q}
$$

with a reliability constant (behind the notation $\lesssim$ ) which does not depend on $\lambda$ or $h_{\mathscr{T}}$. 


\subsubsection{Arnold-Winther Finite Elements}

Arnold and Winther proposed a mixed finite element method with symmetric stress field (cf. [AW02]). These elements are locking-free in numerical experiments and satisfy the predicted convergence rates, cf. [CGRT08].

One seeks the displacement field $u \in H_{0}^{1}\left(\Omega ; \mathbb{R}^{2}\right)$ and the stress tensor

$$
\sigma \in H\left(\operatorname{div} ; \Omega ; \mathbb{R}_{\text {sym }}^{2 \times 2}\right)
$$

satisfying the mixed problem (8.1). We consider the spaces

$$
V:=H\left(\operatorname{div} ; \Omega ; \mathbb{R}_{\mathrm{sym}}^{2 \times 2}\right), \quad Q:=L^{2}\left(\Omega ; \mathbb{R}^{2}\right),
$$

and their discrete counterparts, on any triangle $T \in \mathscr{T}_{\ell}$,

$$
\begin{aligned}
V_{T} & :=\left\{\tau \in P_{3}\left(T ; \mathbb{R}_{\mathrm{sym}}^{2 \times 2}\right) \mid \operatorname{div} \tau \in P_{1}\left(T ; \mathbb{R}^{2}\right)\right\}, \\
Q_{\ell} & :=P_{1}\left(\mathscr{T}_{\ell} ; \mathbb{R}^{2}\right) .
\end{aligned}
$$

Given the data $f \in L^{2}\left(\Omega ; \mathbb{R}^{2}\right)$, the weak formulation of the linear Lamé problem reads: Find $(\sigma, u) \in V \times Q$ such that

$$
\begin{aligned}
\int_{\Omega} \sigma: \mathbb{C}^{-1} \tau d x+ & \int_{\Omega} u \cdot \operatorname{div} \tau d x=0 & & \text { for all } \tau \in V, \\
& \int_{\Omega} v \cdot \operatorname{div} \sigma d x=-\int_{\Omega} f \cdot v d x & & \text { for all } v \in Q .
\end{aligned}
$$

The corresponding discrete solution in the discrete spaces defined above is denoted $\left(\sigma_{\ell}, u_{\ell}\right)$. We refer to Section 3.5 and stress that $\sigma_{\ell}$ is symmetric. The analysis follows that of the previous section and starts with (8.3). The equilibrium residual reads

$$
\mathscr{R} e s_{V}(v):=\int_{\Omega} f \cdot v d x-\int_{\Omega} \varepsilon(v): \sigma_{\ell}=\int_{\Omega}\left(f+\operatorname{div} \sigma_{\ell}\right) \cdot v d x .
$$

Analogous to Section 8.3.1, it can be estimated by $\operatorname{osc}\left(f, \mathscr{T}_{\ell}\right):=\min _{f_{\ell} \in P_{1}\left(\mathscr{T}_{\ell}\right)^{2}}\left\|h_{\mathscr{T}}\left(f-f_{\ell}\right)\right\|$. The consistency residual reads

$$
\left.\mathscr{R} e s_{Q}(\tau)=\int_{\Omega}\left(\varepsilon\left(\tilde{u}_{\ell}\right)\right)-\mathbb{C}^{-1} \sigma_{\ell}\right): \tau d x .
$$

It can be shown that

$$
\mu_{\ell}:=\left\|\mathbb{C}^{-1} \sigma_{\ell}-\varepsilon\left(\tilde{u}_{\ell}\right)\right\|_{L^{2}\left(\mathscr{T}_{\ell} ; \mathbb{R}^{2 \times 2}\right)} \lesssim\left\|\mathbb{C}^{-1} \sigma_{\ell}-\varepsilon\left(A_{\mathscr{T}_{\ell}} u_{\ell}\right)\right\|_{L^{2}\left(\mathscr{T}_{\ell} ; \mathbb{R}^{2 \times 2}\right)} \approx\left\|\mathscr{R} e s_{V}\right\|_{V^{*}}
$$

with any averaging operator $A_{\mathscr{T}_{\ell}}: P_{0}\left(\mathscr{T}_{\ell} ; \mathbb{R}^{2}\right) \rightarrow P_{1}\left(\mathscr{T}_{\ell} ; \mathbb{R}^{2}\right) \cap C\left(\Omega ; \mathbb{R}^{2}\right)$ which reflects the Dirichlet boundary conditions. 


\section{Eddy Current Problem}

\subsection{Conforming Edge Element Methods}

The curl-conforming approximation of the eddy current equation (2.6) by means of the lowest order edge elements of Nédélec's first family [Néd80, Néd86] with respect to a simplicial triangulation $\mathscr{T}_{\ell}$ of the computational domain $\Omega \subset \mathbb{R}^{3}$ amounts to the computation of $u_{\ell} \in \mathrm{Nd}_{1,0}\left(\mathscr{T}_{\ell}\right)$ such that

$$
\int_{\Omega}\left(\mu^{-1} \operatorname{curl} u_{\ell} \cdot \operatorname{curl} v_{\ell}+\sigma u_{\ell} \cdot v_{\ell}\right) d x=\int_{\Omega} f \cdot v_{\ell} d x \quad \text { for all } v_{\ell} \in \operatorname{Nd}_{1,0}\left(\mathscr{T}_{\ell}\right) .
$$

Consequently, in (3.7),(3.8) we may choose $\tilde{u}_{\ell}=u_{\ell}$ and $p_{\ell}=\mu^{-1}$ curl $u_{\ell}$. For the consistency residual $\mathscr{R} e s_{\text {cons }}=\mathscr{R} e s_{Q}$ it follows that

$$
\mathscr{R} e s_{Q}(q)=0 \quad \text { for all } q \in \operatorname{Nd}_{1,0}\left(\mathscr{T}_{\ell}\right) .
$$

On the other hand, the equilibrium residual $\mathscr{R} e s_{\text {eq }}=\mathscr{R} e s_{V}$ reads

$$
\mathscr{R} e s_{V}(v)=\int_{\Omega}\left(f-\sigma u_{\ell}\right) \cdot v d x-\int_{\Omega} p_{\ell} \cdot \operatorname{curl} v d x \quad \text { for all } v \in V .
$$

Following [ABDG98], we may decompose $v \in V$ by means of

$$
v=z+\nabla \varphi \text { for } z \in \operatorname{ker}(\operatorname{curl})^{\perp} \text { and } \varphi \in H_{0}^{1}(\Omega),
$$

so that the equilibrium residual splits accordingly,

$$
\begin{array}{ll}
\mathscr{R} e s_{V}^{(1)}(z):=\int_{\Omega}\left(f-\sigma u_{\ell}\right) \cdot z d x-\int_{\Omega} p_{\ell} \cdot \operatorname{curl} z d x & \text { for } z \in \operatorname{ker}(\text { curl })^{\perp}, \\
\operatorname{Res}_{V}^{(2)}(\varphi):=\int_{\Omega}\left(f-\sigma u_{\ell}\right) \cdot \nabla \varphi d x & \text { for } \varphi \in H_{0}^{1}(\Omega) .
\end{array}
$$

An elementwise application of Stokes' theorem resolves the second integral on the righthand side of (9.1a) to

$$
\int_{T} p_{\ell} \cdot \operatorname{curl} z d x=-\int_{\partial T} v \wedge\left(p_{\ell} \wedge v\right) \cdot(z \wedge v) d \sigma+\int_{T} \operatorname{curl} p_{\ell} \cdot z d x
$$

Hence,

$$
\begin{aligned}
\mathscr{R e s}_{V}^{(1)}(z)= & \sum_{T \in \mathscr{T}_{\ell}} \int_{T}\left(f-\sigma u_{\ell}-\operatorname{curl} p_{\ell}\right) \cdot z d x \\
& +\sum_{F \in \mathscr{F}_{\ell}} \int_{F}\left[v \wedge\left(p_{\ell} \wedge v\right)\right]_{F} \cdot(z \wedge v) d \sigma \text { for } z \in \operatorname{ker}(\text { curl })^{\perp} .
\end{aligned}
$$


On the other hand, for $\varphi \in H_{0}^{1}(\Omega)$ an elementwise Green's formula on the right-hand side in (9.1b) results in

$$
\mathscr{R}_{e}(2)(\varphi)=\sum_{T \in \mathscr{T}_{\ell}} \int_{T} \operatorname{div}\left(\sigma u_{\ell}-f\right) \varphi d x+\sum_{F \in \mathscr{F}_{\ell}} \int_{F}\left[v \cdot\left(f-\sigma u_{\ell}\right)\right]_{F} \varphi d \sigma .
$$

Introducing the element residuals

$$
R_{T}^{(1)}:=\left.\left(f-\sigma u_{\ell}-\operatorname{curl} p_{\ell}\right)\right|_{T} \quad \text { and } \quad R_{T}^{(2)}:=\left.\operatorname{div}\left(\sigma u_{\ell}-f\right)\right|_{T},
$$

and the face residuals

$$
R_{F}^{(1)}:=\left[v \wedge\left(p_{\ell} \wedge v\right)\right]_{F} \text { and } R_{F}^{(2)}:=\left[v \cdot\left(f-\sigma u_{\ell}\right)\right]_{F}
$$

and applying Theorem 4.1 and Theorem 5.2 yield the equilibrium error estimator (cf. [HS09, Sch08])

$$
\eta_{\ell}=\left(\sum_{j=1}^{2}\left(\sum_{T \in \mathscr{T}_{\ell}} h_{T}^{2}\left\|R_{T}^{(j)}\right\|_{L^{2}(T)}^{2}+\sum_{F \in \mathscr{F}_{\ell}} h_{F}\left\|R_{F}^{(j)}\right\|_{L^{2}(F)}^{2}\right)\right)^{1 / 2} .
$$

\subsection{Discontinuous Galerkin Methods}

We consider an Interior Penalty Discontinuous Galerkin (IPDG) method for the eddy current equations (2.6). Given a geometrically conforming, shape-regular simplicial triangulation $\mathscr{T}_{\ell}$ of the computational domain $\Omega \subset \mathbb{R}^{3}$, the discrete spaces $V_{\ell}$ and $Q_{\ell}$ are chosen as elementwise polynomials of degree $\leq p$,

$$
V_{\ell}:=P_{p}\left(\mathscr{T}_{\ell} ; \mathbb{R}^{3}\right) \quad \text { and } \quad Q_{\ell}:=P_{p}\left(\mathscr{T}_{\ell} ; \mathbb{R}^{3}\right)
$$

For $u_{\ell}, v_{\ell} \in V_{\ell}$ and $q_{\ell} \in Q_{\ell}$ we set

$$
\begin{aligned}
& J_{V}\left(u_{\ell}, v_{\ell}\right):=\sum_{F \in \mathscr{F}_{\ell}} \int_{F}\left(\left\{v \wedge\left(\operatorname{curl} u_{\ell} \wedge v\right)\right\}_{F}-\alpha\left[u_{\ell} \wedge v\right]_{F}\right) \cdot\left[v_{\ell} \wedge v\right]_{F} d \sigma \\
& \left.J_{Q}\left(v_{\ell}, q_{\ell}\right):=\sum_{F \in \mathscr{F}_{\ell}} \int_{F}\left\{v \wedge q_{\ell} \wedge v\right)\right\}_{F} \cdot\left[v_{\ell} \wedge v\right]_{F} d \sigma
\end{aligned}
$$

where $\alpha \geq \alpha_{\min }>0$ is some suitably chosen penalty parameter. The mixed formulation of the Interior Penalty Discontinuous Galerkin Method reads: Find $\left(u_{\ell}, p_{\ell}\right) \in V_{\ell} \times Q_{\ell}$ such that

$$
\begin{aligned}
a\left(p_{\ell}, q_{\ell}\right)-b\left(u_{\ell}, q_{\ell}\right)=\ell_{Q}\left(q_{\ell}\right)+J_{Q}\left(u_{\ell}, q_{\ell}\right) & \text { for all } q_{\ell} \in Q_{\ell} \\
b\left(v_{\ell}, p_{\ell}\right)+c\left(u_{\ell}, v_{\ell}\right)=\ell_{V}\left(v_{\ell}\right)+J_{V}\left(u_{\ell}, v_{\ell}\right) & \text { for all } v_{\ell} \in V_{\ell} .
\end{aligned}
$$


We note that the classical formulation in the primal variable reads: Find $u_{\ell} \in V_{\ell}$ such that, for all $v_{\ell} \in V_{\ell}$, there holds

$$
\begin{aligned}
c\left(u_{\ell}, v_{\ell}\right) & +\sum_{T \in \mathscr{T}_{\ell}}\left(\mu^{-1} \operatorname{curl} u_{\ell}, \operatorname{curl} v_{\ell}\right)_{L^{2}(T)} \\
& =\ell_{Q}\left(\mu^{-1} \operatorname{curl} v_{\ell}\right)+\ell_{V}\left(v_{\ell}\right)+J_{Q}\left(u_{\ell}, \mu^{-1} \operatorname{curl} v_{\ell}\right)+J_{V}\left(u_{\ell}, v_{\ell}\right) .
\end{aligned}
$$

Remark 9.1. It is easy to see that the formulations (9.3)-(9.4) are formally equivalent in the following sense. If $\left(u_{\ell}, p_{h}\right) \in V_{\ell} \times Q_{\ell}$ solves (9.3), then $u_{\ell} \in V_{\ell}$ solves (9.4). Conversely, if $u_{\ell} \in V_{\ell}$ solves (9.4), then there exists some $p_{\ell} \in Q_{\ell}$ such that $\left(u_{\ell}, p_{\ell}\right)$ solves (9.3) (cf. Theorem 4.1 in [CHO9]).

The consistency error associated with the solution $\left(u_{\ell}, p_{\ell}\right) \in V_{\ell} \times Q_{\ell}$ of (9.3) is given by

$$
\mu_{\ell}:=\min _{\tilde{v}_{\ell} \in V}\left(\left\|u_{\ell}-\tilde{v}_{\ell}\right\|_{L^{2}(\Omega)}^{2}+\left\|\operatorname{curl}_{\ell} u_{\ell}-\operatorname{curl} \tilde{v}_{\ell}\right\|_{L^{2}(\Omega)}^{2}\right)^{1 / 2}
$$

where $\operatorname{curl}_{\ell}$ stands for the elementwise curl. The minimum is attained with a minimizer $\tilde{u}_{\ell} \in V$, i.e.,

$$
\mu_{\ell}^{2}=\left\|u_{\ell}-\tilde{u}_{\ell}\right\|_{L^{2}(\Omega)}^{2}+\left\|\operatorname{curl}_{\ell} u_{\ell}-\operatorname{curl} \tilde{u}_{\ell}\right\|_{L^{2}(\Omega)}^{2} .
$$

Choosing $p_{\ell}:=\mu^{-1}$ curl $\tilde{u}_{\ell} \in Q$, the residuals $\mathscr{R} e s_{V}$ and $\mathscr{R} e s_{Q}$ are given by

$$
\begin{aligned}
& \mathscr{R e s}_{V}(v)=\int_{\Omega}\left(f \cdot v-\mu^{-1} \operatorname{curl}_{\ell} \tilde{u}_{\ell} \cdot \operatorname{curl} v-\sigma \tilde{u}_{\ell} \cdot v\right) d x, \quad v \in V, \\
& \mathscr{R e s}_{Q}(q)=0, \quad q \in Q .
\end{aligned}
$$

Since $J_{V}\left(u_{\ell}, v_{\ell}\right)=0$ for $v_{\ell} \in \operatorname{Nd}_{1,0}\left(\mathscr{T}_{\ell}\right)$, an application of Stokes' theorem shows, for all $v_{\ell} \in \mathrm{Nd}_{1,0}\left(\mathscr{T}_{\ell}\right)$,

$$
\mathscr{R} e s_{V}\left(v_{\ell}\right)=c\left(u_{\ell}-\tilde{u}_{\ell}, v_{\ell}\right)
$$

The unified theory leads to

$$
\|\left(u-\tilde{u}_{\ell}, p-p_{\ell} \|_{V \times Q} \lesssim \eta_{\ell}+\mu_{\ell}\right.
$$

where the estimator $\eta_{\ell}$ is as in (9.2) with the element and face residuals $R_{T}^{(i)}, R_{F}^{(i)}, 1 \leq i \leq 2$, given by

$$
\begin{aligned}
& R_{T}^{(1)}:=\left.h_{T}\left(f-\sigma u_{\ell}-\operatorname{curl} \mu^{-1} \operatorname{curl} u_{\ell}\right)\right|_{T}, \quad R_{T}^{(2)}:=\left.h_{T} \operatorname{div}\left(f-\sigma u_{\ell}\right)\right|_{T}, \\
& R_{F}^{(1)}:=h_{F}^{1 / 2}\left[v \wedge\left(\mu^{-1} \operatorname{curl} u_{\ell} \wedge v\right)\right]_{F}, \quad R_{F}^{(2)}:=h_{F}^{1 / 2}\left[v \cdot\left(f-\sigma u_{\ell}\right)\right]_{F} .
\end{aligned}
$$

An estimate $\bar{\mu}_{\ell}$ for the consistency error $\mu_{\ell}$ has been provided in Proposition 4.1 of [HPS07] according to

$$
\mu_{\ell}^{2} \lesssim \bar{\mu}_{\ell}^{2}:=\alpha \sum_{F \in \mathscr{F}_{\ell}(\Omega)} h_{F}^{-1}\left\|\left[u_{\ell} \wedge v\right]_{F}\right\|_{L^{2}(F)}^{2}
$$


which yields

$$
\left\|\left(u-\tilde{u}_{\ell}, p-p_{\ell}\right)\right\|_{V \times Q} \lesssim \eta_{\ell}+\bar{\mu}_{\ell} .
$$

As in the case of IPDG for second order elliptic boundary value problems (cf., e.g., Lemma 3.6 in [BN10]), it is not difficult to see that $\bar{\mu}_{\ell}$ can be controlled by the estimator $\eta_{\ell}$. In fact, for sufficiently large penalty parameter $\alpha$ it holds

$$
\alpha\left(\sum_{F \in \mathscr{F}_{\ell}(\Omega)} h_{F}^{-1}\left\|\left[u_{\ell} \wedge v\right]_{F}\right\|_{L^{2}(F)}^{2}\right)^{1 / 2} \lesssim \eta_{\ell}
$$

so that we arrive at

$$
\left\|\left(u-\tilde{u}_{\ell}, p-p_{\ell}\right)\right\|_{V \times Q} \lesssim \eta_{\ell}
$$

\section{References}

[ABD84] D.N. Arnold, F. Brezzi, and J. Douglas. PEERS: A new finite element for plane elasticity. Japan J. Appl. Math, 1(2):347-367, 1984.

[ABDG98] C. Amrouche, C. Bernardi, M. Dauge, and V. Girault. Vector potentials in threedimensional non-smooth domains. Math. Meth. Appl. Sci., 21:823-864, 1998.

[AFW06] D.N. Arnold, R.S. Falk, and R. Winther. Finite element exterior calculus, homological techniques, and applications. Acta Numer., 15:1-155, 2006.

[AFW10] D.N. Arnold, R.S. Falk, and R. Winther. Finite element exterior calculus: from hodge theory to numerical stability. Bull. Amer. Math. Soc., 47:281-354, 2010.

[AO00] M. Ainsworth and J.T. Oden. A posteriori error estimation in finite element analysis. Wiley-Interscience [John Wiley \& Sons], New York', 2000.

[AW02] D.N. Arnold and R. Winther. Mixed finite elements for elasticity. Numer. Math., 92:401-419, 2002.

[BCR04] D. Braess, C. Carstensen, and B.D. Reddy. Uniform convergence and a posteriori error estimators for the enhanced strain finite element method. Numer. Math, 96:461-479, 2004.

[BDD04] P. Binev, W. Dahmen, and R. DeVore. Quasi-optimal convergence rate of an adaptive discontinuous galerkin method. Numer. Math., 97:219-268, 2004.

[BF91] F. Brezzi and M. Fortin. Mixed and Hybrid Finite Element Methods. Springer-Verlag, New York, 1991.

[BHHW00] R. Beck, R. Hiptmair, R.H.W. Hoppe, and B. Wohlmuth. Residual based a posteriori error estimators for eddy current computation. $M^{2}$ AN Math. Modeling and Numer. Anal., 34:159-182, 2000.

[BN10] A. Bonito and R. Nochetto. Quasi-optimal convergence rate of an adaptive discontinuous galerkin method. SIAM J. Numer. Anal., 48:734-771, 2010.

[Bra07] D. Braess. Finite Elements: Theory, Fast Solvers, and Applications in Solid Mechanics. Cambridge University Press, 3rd edition, 2007.

[BS94] S.C. Brenner and L.R. Scott. The mathematical theory of finite element methods, volume 15 of Texts in Applied Mathematics. Springer Verlag, New York, 1994.

[BS01] I. Babuška and T. Strouboulis. The Finite Element Method and its Reliability. The Clarendon Press, Oxford University Press, 2001.

[Car97] C. Carstensen. A posteriori error estimate for the mixed finite element method. Math. Comp., 66:465-476, 1997. 
[Car99] C. Carstensen. Quasi-interpolation and a posteriori error analysis in finite element method. M2AN Math. Model. Numer. Anal., 33(6):1187-1202, 1999.

[Car05] C. Carstensen. A unifying theory of a posteriori finite element error control. Numer. Math., 100(4):617-637, 2005.

[CD98] C. Carstensen and G. Dolzmann. A posteriori error estimates for mixed FEM in elasticity. Numer. Math., 81(2):187-209, 1998.

[CDFH00] C. Carstensen, G. Dolzmann, S.A. Funken, and D.S. Helm. Locking-free adaptive mixed finite element methods in linear elasticity. Comput. Methods Appl. Mech. Engrg., 190(13-14):1701-1718, 2000.

[CGRT08] C. Carstensen, D. Günther, J. Reininghaus, and J. Thiele. The Arnold-Winther mixed FEM in linear elasticity. Part I: Implementation and numerical verification. Comput. Methods Appl. Mech. Engrg., 197:3014-3023, 2008.

[CH07] C. Carstensen and J. Hu. A unifying theory of a posteriori error control for nonconforming finite element methods. Numer. Math., 107(3):473-502, 2007.

[CH09] C. Carstensen and R.H.W. Hoppe. Unified framework for an a posteriori error analysis of non-standard finite element approximations of $H$ (curl)-elliptic problems. $J$. Numer. Math., 17:27-44, 2009.

[Cia88] P.G. Ciarlet. Mathematical Elasticity, Vol. I : Three-Dimensional Elasticity. Studies in Mathematics and its Applications. SIAM, 1988.

[Cia02] P.G. Ciarlet. The Finite Element Method for Elliptic Problems. SIAM, 2002.

[CKNS08] J.M. Cascon, Ch. Kreuzer, R.H. Nochetto, and K.G. Siebert. Quasi-optimal rate of convergence of adaptive finite element methods. SIAM J. Numer. Anal., 46:25242550, 2008.

[Clé75] C. Clément. Approximation by finite element functions using local regularization. RAIRO Analyse Numérique, 9:77-84, 1975.

[COH07] C. Carstensen, A. Orlando, and J. Hu. Framework for the a posteriori error analysis of nonconforming finite element. SIAM J. Numer. Anal., 45(1):68-82, 2007.

[Dör96] W. Dörfler. A convergent adaptive algorithm for Poisson's equation. SIAM J. Numer. Anal., 33:1106-1124, 1996.

[HPS07] P. Houston, I. Perugia, and D. Schötzau. A posteriori error estimation for discontinuous Galerkin discretizations of $H$ (curl)-elliptic partial differential equations. IMA Journal of Numerical Analysis, 27:122-150, 2007.

[HS09] R.H.W. Hoppe and J. Schöberl. Convergence of adaptive edge element methods for the 3d eddy currents equations. J. Comp. Math., 27:657-676, 2009.

[KS95] R. Kouhia and R. Stenberg. A linear nonconforming finite element method for nearly incompressible elasticity and stokes flow. Comput. Methods Appl. Mech. Engrg., 124(3):195-212, 1995.

[Mon03] P. Monk. Finite Element Methods for Maxwell's equations. Clarendon Press, Oxford, 2003.

[Néd80] J.-C. Nédélec. Mixed finite elements in $\mathbb{R}^{3}$. Numer. Math., 35:315-341, 1980.

[Néd86] J.-C. Nédélec. A new family of mixed finite elements in $\mathbb{R}^{3}$. Numer. Math., 50:57-81, 1986.

[PW60] L.E. Payne and H.F. Weinberger. An optimal poincaré inequality for convex domains. Numer. Math., 5:286-292, 1960.

[Sch08] J. Schöberl. A posteriori error estimates for Maxwell equations. Math. Comp., 77:633649, 2008.

[Ste07] R. Stevenson. Optimality of a standard adaptive finite element method. Found. Comput. Math., 7:245-269, 2007. 
[Ver96] R. Verfürth. A review of a posteriori error estimation and adaptive mesh-refinement techniques. Wiley-Teubner, 1996.

[WH99] B. Wohlmuth and R.H.W. Hoppe. A comparison of a posteriori error estimators for mixed finite element discretizations. Math. Comp., 82:253-279, 1999.

$\left[\mathrm{ZCS}^{+} 10\right]$ L. Zhong, L. Chen, S. Shu, G. Wittum, and J. Xu. Quasi-optimal convergence of adaptive edge finite element methods for three dimensional indefinite time-harmonic Maxwell's equations. Tech. Rep., Department of Mathematics, University of California at Irvine, 2010. 